\title{
Research Commons
}

http://researchcommons.waikato.ac.nz/

\section{Research Commons at the University of Waikato}

\section{Copyright Statement:}

The digital copy of this thesis is protected by the Copyright Act 1994 (New Zealand).

The thesis may be consulted by you, provided you comply with the provisions of the Act and the following conditions of use:

- Any use you make of these documents or images must be for research or private study purposes only, and you may not make them available to any other person.

- Authors control the copyright of their thesis. You will recognise the author's right to be identified as the author of the thesis, and due acknowledgement will be made to the author where appropriate.

- You will obtain the author's permission before publishing any material from the thesis. 
THE UNIVERSITY OF

WAIKATO

Te Whare Wānanga o Waikato

\section{Live Television in a \\ Digital Library}

\section{Maxime Roüast}

This thesis is submitted in partial fulfillment of the requirements for the

Degree of Master of Science at the University of Waikato.

October 2011

(C) 2011 Maxime Roüast 
2/124 


\begin{abstract}
Nowadays nearly everyone has access to digital television with a growing number of channels available for free. However due to the nature of broadcasting, this huge mass of information that reaches us is not, for the main part, organised-it is principally a succession of images and sound transmitted in a flow of data. Compare this with digital libraries which are powerful at organising a large but fixed set of documents.

This project brings together these two concepts by concurrently capturing all the available live television channels, and segments them into files which are then imported into a digital video library. The system leverages off the information contained in the electronic program guide and the video recordings to generate metadata suitable for the digital library. By combining these two concepts together this way, the aim of this work is to look beyond what is currently available in the digital TV set top boxes on the market today and explore the full potential-unencumbered by commercial market constraints-to what the raw technology can provide.
\end{abstract}




\section{Contents}

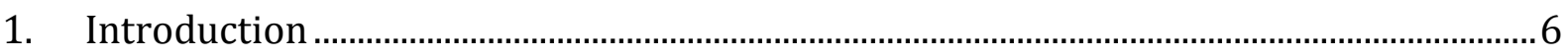

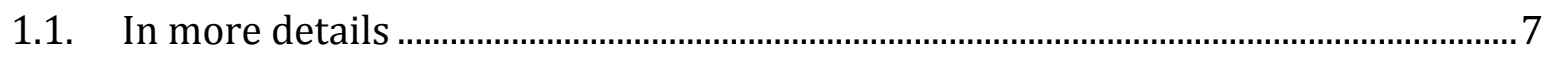

1.2. Replay Me! simplified diagram ……………………...................................................

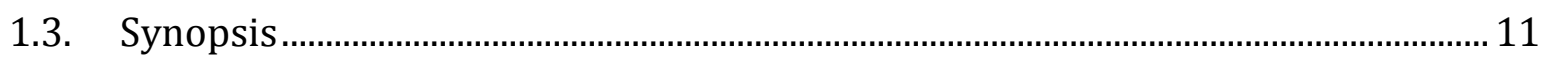

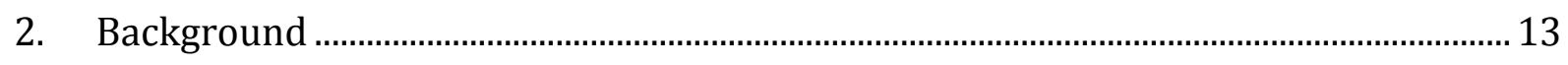

2.1. Recent television trends .............................................................................................. 13

2.2. Digital video analysis and retrieval......................................................................... 15

2.3. Existing commercial approaches ............................................................................. 17

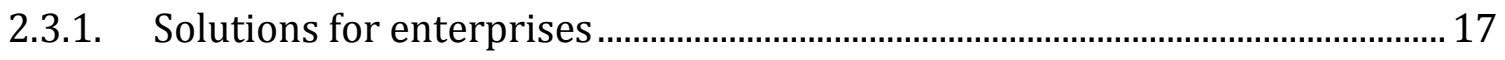

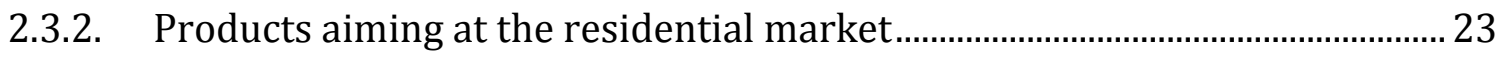

2.4. Existing video digital libraries research projects ........................................................27

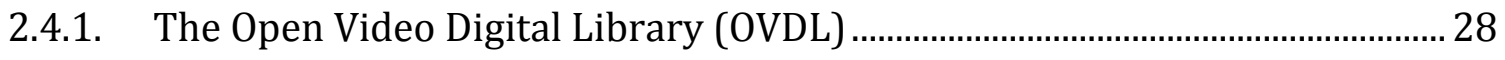

2.4.2. Informedia Digital Video Library …………………………………………….. 32

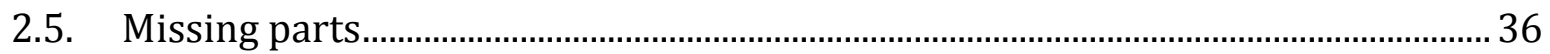

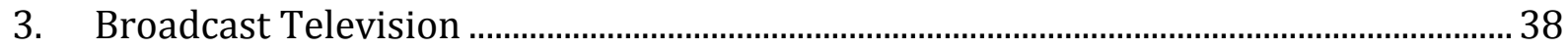

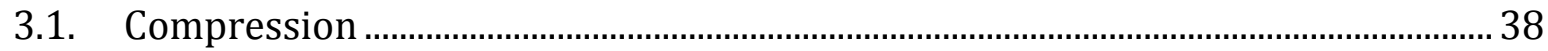

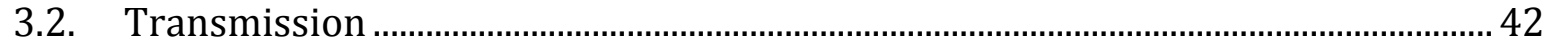

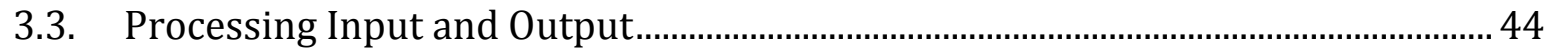

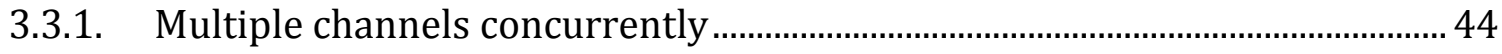

3.3.2. Real time operation and hardware limits ........................................................... 49

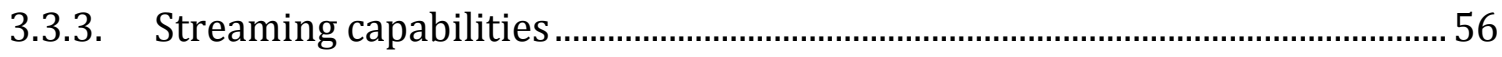

4. Television recording implementation details.................................................................59

4.1. Media Portal TV Server Plugin .................................................................................... 59

4.2. Listing the possible concurrent recorded channels ................................................6 60

4.3. Getting access to the EPG off-the-air ........................................................................62 
4.4. Automatic video segmentation

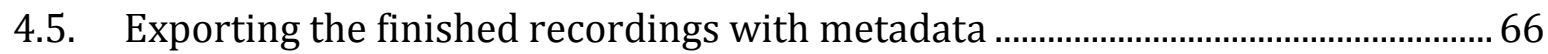

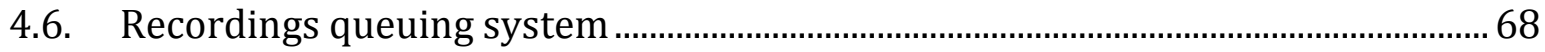

4.7. Recording schedules management and disk space optimisation.............................. 70

4.8. Advanced configuration and activity reporting ….................................................... 73

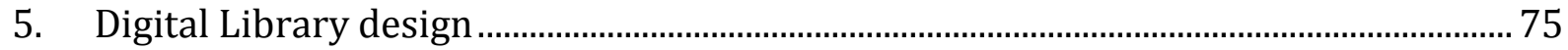

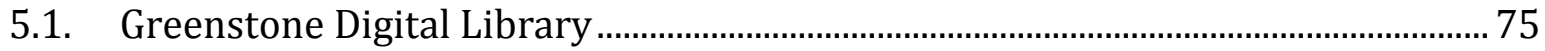

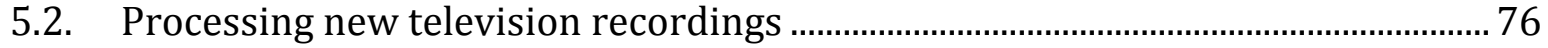

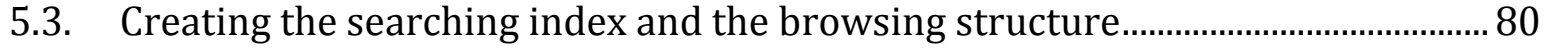

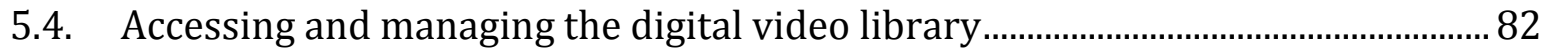

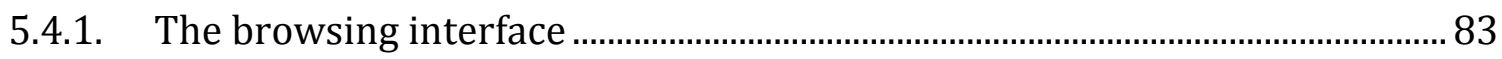

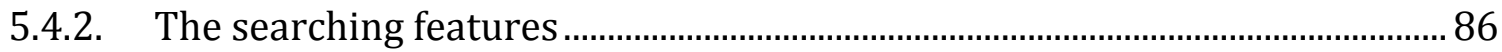

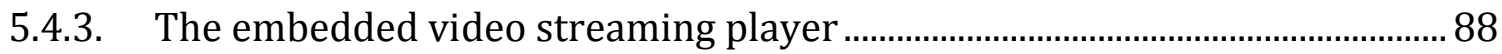

5.4.4. Adding user-edited metadata and extra features ................................................ 91

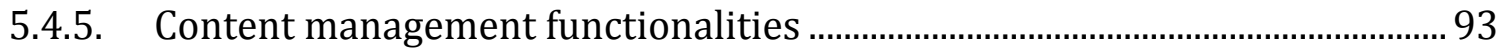

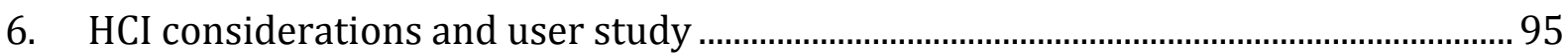

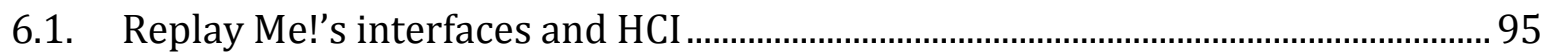

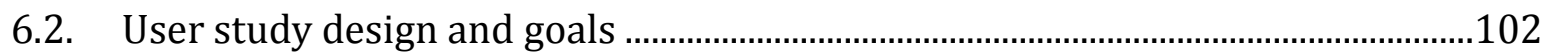

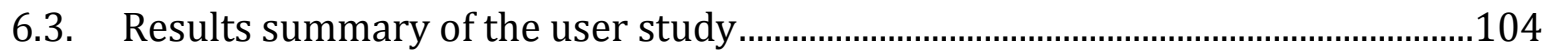

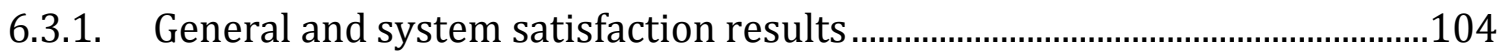

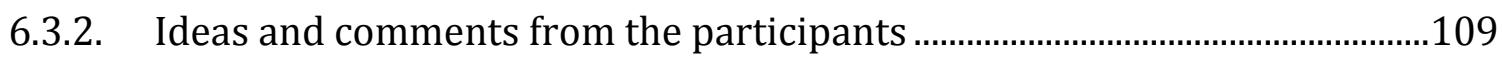

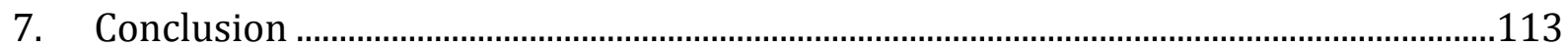

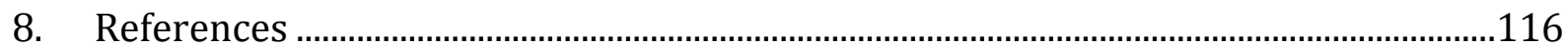




\section{Introduction}

Watching television on demand is increasingly common in people's homes (Elsweiler, Mandl, \& Kirkegaard Lunn, 2010). Solutions range from Internet based catch-up TV services such as TVNZ On Demand or iSKY to more closely integrated TV solutions such as Tivo or MySKYHDi. Each of these solutions enrich the viewer experience, however there are also commercial factors involved that mean there are also deliberate limitations in what is provided. For example, the Tivo system does not allow the recorded content to be directly viewed (streamed) from another television or computer inside the same house. It also requires a broadband service to receive the 14 days "enriched version" of electronic program guide (EPG) to operate, even if this information is available for 7 days in a basic form directly from the TV signal. Moreover, the digital video recorder (DVR) MySKYHDi does not allow the subscriber to backup their recordings for later watching, nor does it allow them to add additional disk space to the system to extend the recording capabilities of the machine-something that is rather frustrating given the increase in the number of channels broadcasted in high definition (HD).

The aim of this project is to look beyond what is currently available on the market today and explore the full potential to what the raw technology can provide. The context to this exploration is to see what can be developed using a regular desktop PC based in a person's home.

In undertaking this work, inspiration was taken from the field of digital library research, and a prototype system called Replay Me! (RM) was developed, which provides a usercentric media centre built from a digital library software architecture that is connected to a live stream of multiple television signals. The key concept to RM is that a user need never think about what to record ahead of time as with other systems. Even in the latest integrated TV systems such as the ones mentioned above, the "series-link" functionthat allows a person to automatically record an entire series of shows-requires the user to proactively record at the initial stage. Also, to many subscribers' chagrin, the series link information can contain errors that result in missed recordings. In comparison, with the approach RM takes it is always recording, maintaining a shifting 
time-based "window" of every TV show that has aired in the last month, ${ }^{1}$ and uses the features of the digital library (DL) to provide access to-and manage over time-this rich resource of TV content.

\subsection{In more details}

The aim of this project is to create a prototype system developed along the lines just described, and then evaluate it to see if it helps people to get better control of the increasing number of digital video content available over the free-to-air television broadcasts.

In order to achieve this goal, all the end user's favourite television channels are recorded simultaneously. There is not any limitation on the number of channels to be recorded at the same time. Moreover neither the technology employed for broadcasting (satellite, terrestrial) nor the video quality (standard definition, high definition) should be an issue for continuous recording. Actually, we are interested in keeping all the content of multiple channels in order to present it in an indexed fashion instead of the traditional television's linear mode.

In this envisaged system, this large quantity of video content is automatically indexed inside a digital library to allow, later on, the user to select the desired program to watch at a suitable time. In fact, this system frees the user from the schedules imposed by broadcasters and to put the user back in control of the choice of programs being offered since everything is available locally, on demand, with no loss of quality.

Due to the large number of videos being recorded every hour, every day, it becomes important to provide the end user with a set of tools to facilitate the searching of interesting content. This is where the digital library component excels: to index and present the video recordings. While it would be nice to keep all the recordings forever, in reality, because of the large amount of disk space required for such a system, and since this system is designed to operate with residential grade equipment to reduce the cost of the hardware requirements to the minimum, the videos are not destined to be kept indefinitely unless manually marked as "keep" through the interface. As a result,

\footnotetext{
${ }^{1}$ One month of recordings is an ideal figure however depending on the number of channels available,
} today's residential computers may not have sufficient disk space to allow a time frame longer than a week. 
only a "time window" (a few days, weeks or more) of video content is stored on disk until they reach their expiration date and get automatically deleted by the system to free up disk space for future recordings.

This is similar in the way that Video On Demand (VOD) services operate but this system still allows the customisation of the time frame's duration used to keep the recordings. Additionally, the system makes available every single show on all of the recorded channels which is very different to VOD systems where broadcasters only select a handful of programmes to upload to their platforms, typically with a time delay of a day. In other cases broadcasters may decide not to offer any VOD services for certain channels or only available to subscription customers such as the free-to-air channel Prime in New Zealand, which is only available on SKY's catch-up TV service (iSKY).

This project aims to be as independent as possible: to be able to operate in any possible television enabled environments, specifically where a land line or broadband access is expensive or simply not existent. Only the feed coming from the television antenna is required for the system to record and present the videos to the user, therefore it can be used in very remote areas. This is significantly different from the VOD and catch-up television systems where Internet access is indispensable for their service to reach their spectators. This system is also different to some smart hard disk recorders such as Tivo which requires connection to the Internet or to a phone line at all times in order to operate (Paula Wilson, 2005; Hybrid Television Services (ANZ) Pty Limited, 2011).

The following requirements were adopted:

- Continuous recording of all TV channels,

- Equipment and interface suitable for home environment,

- Automated organisation of continuous TV streams,

- Manual management of collected TV shows,

- Multiple playback whilst still recording,

- Full services available within home and reduced set of services available off-site.

These requirements are satisfied with a single desktop PC. In Chapter 3, the technical details of how to achieve this are provided. 


\subsection{Replay Me! simplified diagram}

Figure 1.1 provides an overview of the Replay Me! system that was developed through the course of this study. The three key components are: the Antenna, the Electronic Program Guide and the Greenstone digital library software.

The Antenna is the source of all the content that is used in this project: this can be a terrestrial antenna, a satellite dish, a cable TV source or even IPTV coming from an ADSL or fibre connected box. The EPG provides information that is used to allow RM to automatically segment the different television channels. It also contains metadata that is used when the content will be processed by the digital library software. Greenstone is a multilingual, multi-platform and open source digital library software produced by the New Zealand Digital Library Project at the University of Waikato. It was selected for this project primarily because of its flexible architecture but also because of the available on hand programmer expertise.

Individual channels are continuously de-multiplexed from the video source which RM segments as individual video recordings. In fact, each show is pre-recorded by 3 minutes and post-recorded by 5 minutes in order to make sure that the entire program is recorded even if the broadcaster is not exactly on schedule. There is also a blacklist of recordings that filter the TV shows that should never be recorded; such as advertising programmes (often called "infomercials") and other unwanted shows like the waiting loops used by the channels that do not broadcast 24 hours per day.

Once finished recording, each individual raw video recording is sent to Greenstone for processing, along with associated metadata that came from the EPG such as the title, descriptions, airing date/time, and the channel name. At this stage the content of the video file is processed in order to extract additional video metadata that is used to determine:

- The quality of the video (standard or high definition);

- The number of audio channels (some channels are broadcasted in stereo, some in 5.1 surround sound, sometimes in different languages);

- The presence of subtitles (some channels feature subtitles for the hard of hearing);

- The total video duration;

- The file size and other technical information. 


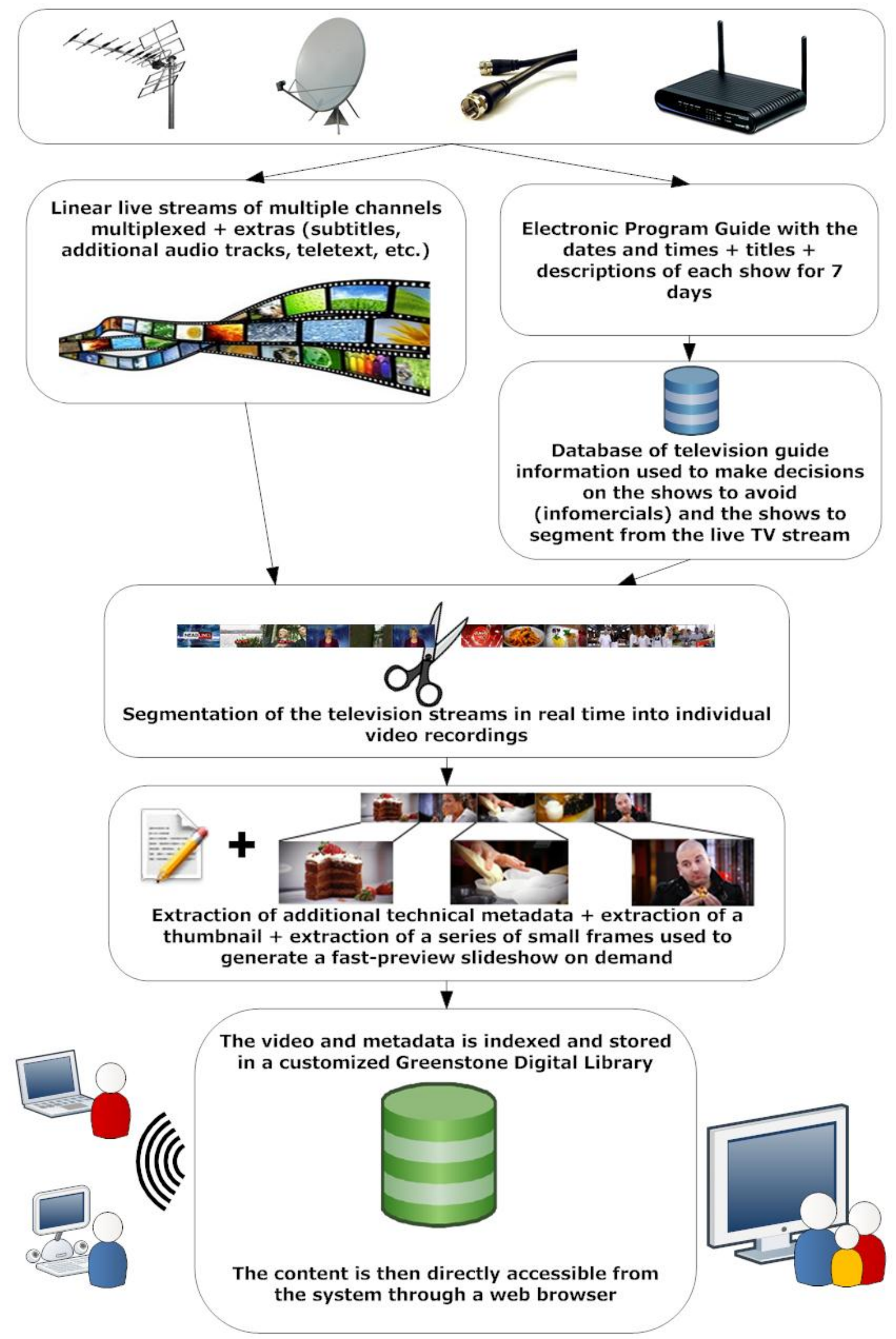

Figure 1.1

"The simplified architecture and deployment of Replay Me!" 
As part of this processing, a large and a small thumbnail is extracted from the video file at approximately $35 \%$ of the way in to the recording. This value was chosen after some experimentation to increase the chance of getting an interesting image describing the content. Following this, a series of small pictures are extracted from the video at regular intervals to create a fast preview slideshow activated on user request. All this extra data is used in the web browser based graphical user interface (GUI) to provide the end user with the maximum details about each video to facilitate searching, browsing and watching.

The video recording along with these extracted graphical and textual metadata elements are then indexed and stored into Greenstone digital library, which will automatically update its database and web interface to reflect the new video entry.

The video library is accessible directly on the host computer which could be in the living room connected to, say, the main television allowing users to browse and watch their selected program at their own pace. Since the system is designed to be a server/client based architecture, it is also possible for other computers connected to the home network to take advantage of RM since they can directly stream different television recordings from the host machine simultaneously.

\subsection{Synopsis}

The structure of the thesis is as follows: in the Chapter 2, past and current related work (both academic and commercial) are identified and reviewed. For instance, projects aiming at creating large digital video libraries from curated data, attempts at understanding user information needs in the context of television watching, the development of Video On Demand services worldwide and the implications for the viewers, the democratisation of "smart" personal digital video recorders, and the emergence of large television recorders targeting government agencies or education providers are described. Then, the similarities and differences with RM are highlighted and the missing parts of each system are discussed in relation to this project.

Chapter 3 explains the technology used to efficiently compress the video from television channels. With the development of high definition channels, new video compression formats were developed to cope with the increasing volume of data required for storage 
and transmission. Next, the techniques used for digital television broadcasting are described along with the challenges of managing such a large volume of incoming data in terms of the speed required for disk storage or network transmission for streaming.

Chapter 4 and Chapter 5 describe the details of RM's operation and specifically the technical challenges associated with this novel project both from the recording side and from the digital library side. As a matter of fact, with the use of large distributed computer systems using the fastest enterprise grade CPU, and an enormous storage space with large professional hard disk arrays such as the ones used in today's datacentres, a system that can perform the tasks of this project can easily be built. However the goal of this research is to be able to create a system that can readily be available to the general public using only a domestic desktop computer, large residential grade hard disks and open source software. With RM recording multiple television channels simultaneously, the real time aspect during operation is critical to avoid an exponential queue of recordings waiting to be processed. Moreover, this chapter explains how additional challenges posed by the fact that all the video content is coming from live streams instead of-for a digital library—the more usual fixed set of curated recordings, have been solved.

In Chapter 6, the details of the user study that was undertaken is explained. Then all the results from this study are analysed in the light of human computer interaction and leisure television watching. These results help provide some insight into the effects watching TV in this new way had, and the different levels of satisfaction experienced by the participants.

The final chapter concludes with a summary of our achievements, and the improvements made to the Greenstone Digital Library software. Then we reflect on the possible paths for future work related to this study. 


\section{Background}

With the increasing amount of information "born digital", its rapid development and availability has changed the way information is accessed. While these changes allow more people to get access to more content anytime from anywhere, it has brought new challenges since the knowledge organisation model and the approach to retrieve the information from electronic environments has had to be redesigned.

In this chapter, the recent developments and trends in television are first discussed, then the need to organise television content is brought into focus in order to introduce some key concepts built upon in this thesis. Finally past and current work both academic and commercial, linked to video digital libraries are identified and reviewed.

\subsection{Recent television trends}

In many western countries, television is still one of the most popular leisure-time pursuits. Surveys indicate that Europeans, for example, watch on average 24 hours of television per week (TNS Gallup, 2010; Kommission für Zulassung und Aufsicht, 2009). Furthermore, there is a recent shift in the way people watch TV, with the increase of channels being broadcast and the volume of content available; in addition to the repeated programmes and new technologies available to record content (Kommission für Zulassung und Aufsicht, 2009).

Meanwhile it has also been observed that with this increase in choice, people are often unsure of what content is available or they have some difficulty in deciding what to watch (Muniz de Ávila \& Donizetti Zorzo, 2009). As a result of this issue, there have been several attempts to build "smart" electronic program guides to help users find content of interest (Ludwig \& Mandl, 2010; Zimmerman, 2004; Muniz de Ávila \& Donizetti Zorzo, 2009; Cotter \& Smyth, 2000).

Studies have demonstrated that-in a leisure environment-people generally want to record content for entertainment or edutainment in addition to bringing their knowledge up-to-date with information such as news, and weather. (Nahrstedt, 2000). More recent studies suggest that, ultimately, users should not be required to proactively look for programmes as with current recording solutions, where this activity dominates 
the interface (Ludwig \& Mandl, 2010; Zimmerman, 2004; Muniz de Ávila \& Donizetti Zorzo, 2009; Cotter \& Smyth, 2000). Instead, it has been suggested that systems which gives the user the possibility to filter content based on a wide range of attributes may be beneficial (Schraefel, et al., 2005; Yee, et al., 2003).

Some steps have been taken towards lowering the active role a user must take in recording shows. Television has evolved significantly over the last 5 years and continues to evolve, and people nowadays can mostly forget about schedules and channels with the introduction of new services such as video on demand into homes. Indeed, sometimes these solutions do not even resemble the traditional TV set up. Still, bringing the Internet connected PC to the television is not always a straightforward process. Solutions such as Tivo, Apple TV, Google TV and other similar platforms could help, but it will take time before these devices and services will become ubiquitous. Google TV is notable in that sales in its first year were disappointing, leading manufacturers to slash prices. In fact, a key obstacle to adoption in many households to these types of advanced TV solutions is the usage caps imposed upon the consumer through Internet subscriptions (Levy, 2010).

The sector is in transition, and the future configuration TV will have is far from clear. In Canada, PricewaterhouseCoopers analysts foresaw a growth in subscription fees of $6.8 \%$ annually in the market, largely due to the growth in VOD services, IP-based television and other digital cable services. However, this is a relatively new market, and according to the vice-president of video products from Rogers Communication-with over 2.3 million subscribers in Canada-it is not yet the time to shut down completely the traditional TV channels as "there are still a large number of people who like to scan through channels to pick something" (Levy, 2010).

Nonetheless, the VOD market has demonstrated a similar growth in many developed countries. For instance, in United Kingdom, BBC programmes have been made available on demand for 7 days after airing to the public through their iPlayer Internet service since 2007. This was in response to their competitor, Channel 4, which started to provide on demand access 5 months earlier with over 20 million programmes and other content being viewed during this period. This clearly demonstrates that people do not want to be tied to broadcast schedules (Sabbagh, 2007). 


\subsection{Digital video analysis and retrieval}

We live in the era of "information explosion" which means that information is created and accessed in a wide range of formats that combine of text, graphics, audio and digital videos on a large scale. To enrich the user experience, authoring and searching tools have been developed that take advantage of this increasing volume of multimedia content (Chang, et al., 1997). To date, the most efficient methods in terms of accuracy for searching within the video medium is to use text-based techniques, often involving manual efforts to write transcripts and other text annotations which is prohibitively expensive for very large collections (Bollen, 2007; Grossmman \& Frieder, 1998; Ioannou, et al., 2000; Shyu, et al., 2003; Sun, et al., 2003). However, new techniques that allow the complete automation of this process are being actively explored in recent research projects, which we will come back later in this chapter.

Similarly, the bourgeoning area of video on demand brings into focus the issues of efficient management, retrieval and rapid video viewing. In reality, VOD systems are about finding mechanisms to access video content often from enormous amounts of data. According to Bolle et al., they should allow the viewers to easily filter out unwanted information instead of being forced to watch video in a linear fashion (Bolle, et al., 1998).

Visual summaries are an interesting solution to help viewers quickly select a video of interest from a library as they give an idea of what a candidate video is about. They also permit random video access since it gives the user clues on the visual content and possibly the length of the video (Bolle, et al., 1998). As a result, a synergy between textbased and visual searching is beneficial since they both indicate a different description of the same content, however there is still room for improvement in this area (Chang, et al., 1997). Furthermore, visual summaries also play a key role for bandwidth optimisation in digital video environments. In fact, they are usually in low resolution so users can browse with pictorial descriptions quickly, whilst still keeping their bandwidth usage down, before finalising their video selection to watch in high resolution (Chang, et al., 1997). 
Video processing algorithms that generate "intelligent" video annotations are not perfect, however, and they have high computing requirements, in some cases taking longer than real-time to process a video (Bolle, et al., 1998). 


\subsection{Existing commercial approaches}

The development of digital video broadcasting has brought many new opportunities for commercial applications. There are mainly two principal markets for these solutions, one is aimed at large organisations, governmental agencies or educational institutions and the other is targeting the wider public.

Two related commercial solutions aimed at enterprises or educational institutions are first analysed, then some widely adopted products targeting the individual consumer market are reviewed.

\subsubsection{Solutions for enterprises}

\section{SnapStream Server}

In North America, SnapStream Media-an innovative company developing digital multimedia entertainment software based in Houston, Texas and originally known in the personal video station (PVR) market with products similar to Microsoft Windows Media Centre-has developed a product aimed at the business market called "SnapStream Server." This system is described to be "a cross between a digital video recorder and a search engine" (Patel, 2009).

In a SnapStream installation, one rack mounted server is capable or recording 10 channels simultaneously and uses the closed captioning that is required by the U.S. Federal Communications Commission (FCC) since 1996, broadcast with each program (Federal Communications Commission, 2011). Recordings are accessible from external computers connected to the SnapStream Server via the network using their proprietary client software designed for Microsoft Windows. Users can search the collection of video recordings by title or description; in fact this system goes beyond this basic capability using the closed caption data to match a keyword to an exact position within a video recording.

As noted by Richard Doherty-director of research at New York based consulting firm, Envisioneering Research-SnapStream Server was one of the first systems to exploit closed captioning (Patel, 2009). 
The SnapStream system is not cheap even for enterprises. The cost for this HD compatible solution at the time of writing starts at NZ \$55,500 (US \$48,500) providing one server, 5 HD tuners, 25 client licences, 9TB of storage space-and can go up to NZ $\$ 114,500+$ (US $\$ 100,000+$ ) depending on the options selected. The company also takes $15 \%$ of the total configuration price as a yearly maintenance fee (Snap Stream, 2011). Moreover, the system is currently designed only for the North American broadcasting standards (Dickson, 2009). It does not natively support satellite or cable television without external cable or satellite setup boxes as it works internally using ATSC and QAM tuners (U.S. compatible digital television equipment) for recording (Snap Stream, 2011). Nevertheless, their solution is completely scalable as a set of servers can be easily arranged into a cluster, as pictured in Figure 2.1, thus multiplying the capabilities of the system. In terms of copyright, correct usage is the responsibility of the client.

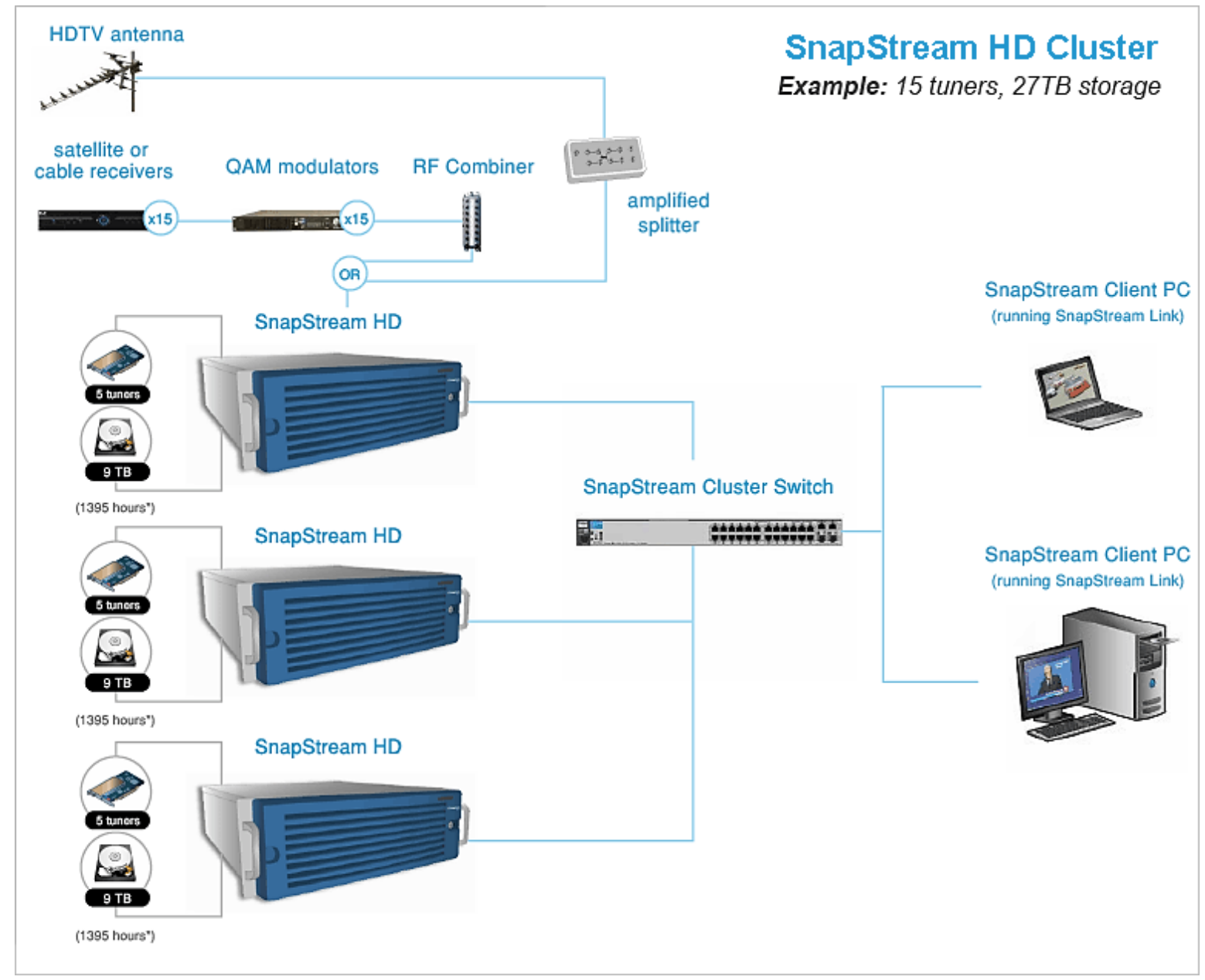

Figure 2.1

"SnapStream Server arranged in a cluster-Reproduced (Snap Stream, 2011)" 
The company's enterprise product was released in 2007. Some of their customers include Hillary Clinton's 2008 presidential campaign and other political committees, Huston Police Department, the U.S. Senate, the University of Missouri, Emerson College, Entertainment Television, the National Broadcasting Company (NBC), the Disney-owned American Broadcasting Company in New York (WABC), Comcast Entertainment, and many other large corporations. Paul Niwa, a professor of journalism in Emerson College has taught undergraduate and postgraduate students using this tool since 2008. He argues that the SnapStream approach "present major implications for the future of how we consume television, and how broadcast journalists approach their work" by making them more accountable for what they say for instance (Fenwick, 2010).

In 2010 SnapStream Enterprise built one of their largest servers ever, made to demonstrate their technology to future clients at the National Association of Broadcasters Show in Las Vegas. This enormous digital video recording station of a size similar to a refrigerator as shown in Figure 2.2, and is capable of recording up to 50 channels simultaneously. In order to achieve this, a total of 50 tuners have been installed into 5 servers powered by 8 Xeon Processors and 68 hard drives of 2TB capacity, totalling 102TB of raid storage.

SnapStream Media also demonstrated their first prototype compatible with the European standard also called DVB-T-which is the same as is used in New Zealandduring the International Broadcasting Convention (IBC) in mid-September 2011 at Amsterdam.

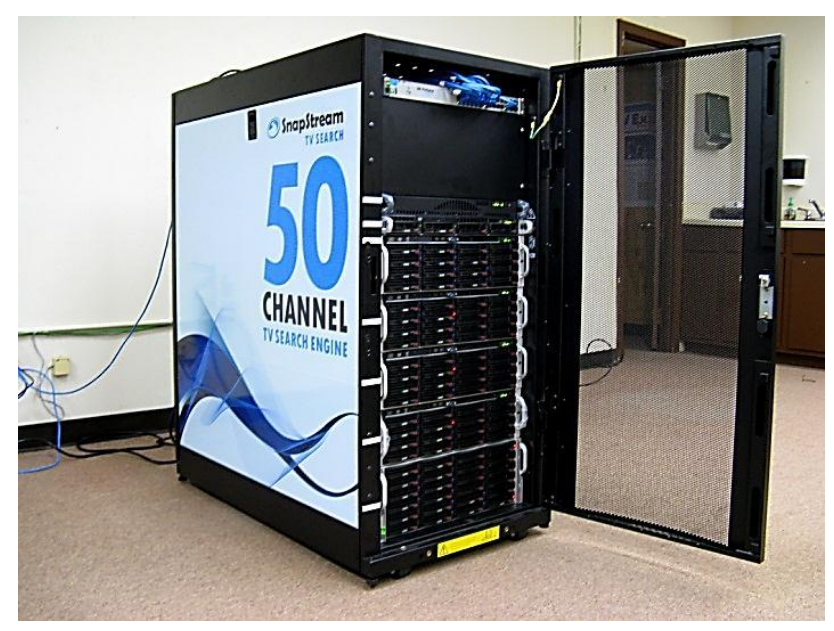

Figure 2.2

“SnapStream's largest DVR-Reproduced (Snap Stream, 2011)” 


\section{Planet eStream}

Planet PC is a company based in United Kingdom which focuses on delivering hardware and software solutions for video acquisition, editing, archiving, streaming and other distribution types mainly to large organisations and the educational sector. One of their product offerings is called the "Planet eStream Suite" which is well known by U.K. educational institutions. This product comes as a core solution to which one can add modules, depending on the needs of the customer. In a nutshell, this software suite is capable of live video streaming and archiving, capturing academic lectures and recording free-to-air television on the Freeview platform (Planet Enterprises Ltd, 2011).

The core component of the software is dedicated to the archiving and streaming of digitized video and audio content which is then made available to an unlimited number of clients via a web interface. It allows administrators to upload new content, create categories, add metadata, create chapter points and playlists directly from their web browser. The search engine lets users find the content they were looking for from the video library by typing keywords or phrases. The developers argue that using this product can benefit "examination revisions, continuing professional development for teaching and other staff, absent students and distance learning for excluded students" (Planet Enterprises Ltd, 2011).

Their "Freeview Recording Module" allows any programme broadcasted on the free-toair platform to be recorded with a single click from the electronic programme guide as pictured in Figure 2.3. As of 2011, the latest revision of their software is now capable of constantly recording all the channels specified by administrators and lets users choose from this pool of recordings the programmes they wish to add to the main library for archiving. This feature is also known as "retrospective TV programme recording" (Planet Enterprises Ltd, 2011). By default one server can record up to 25 channels simultaneously but the system is scalable and this limitation can easily be overcome by ordering a larger server and more disk space. In July 2011, they also improved the eStream Freeview Recording module by enhancing the Series-Link function, allowing users to watch live television directly from their web browser across the local network of their educational provider (Planet PC, 2011). However, at time of writing this system is not yet compatible with satellite television feeds. 


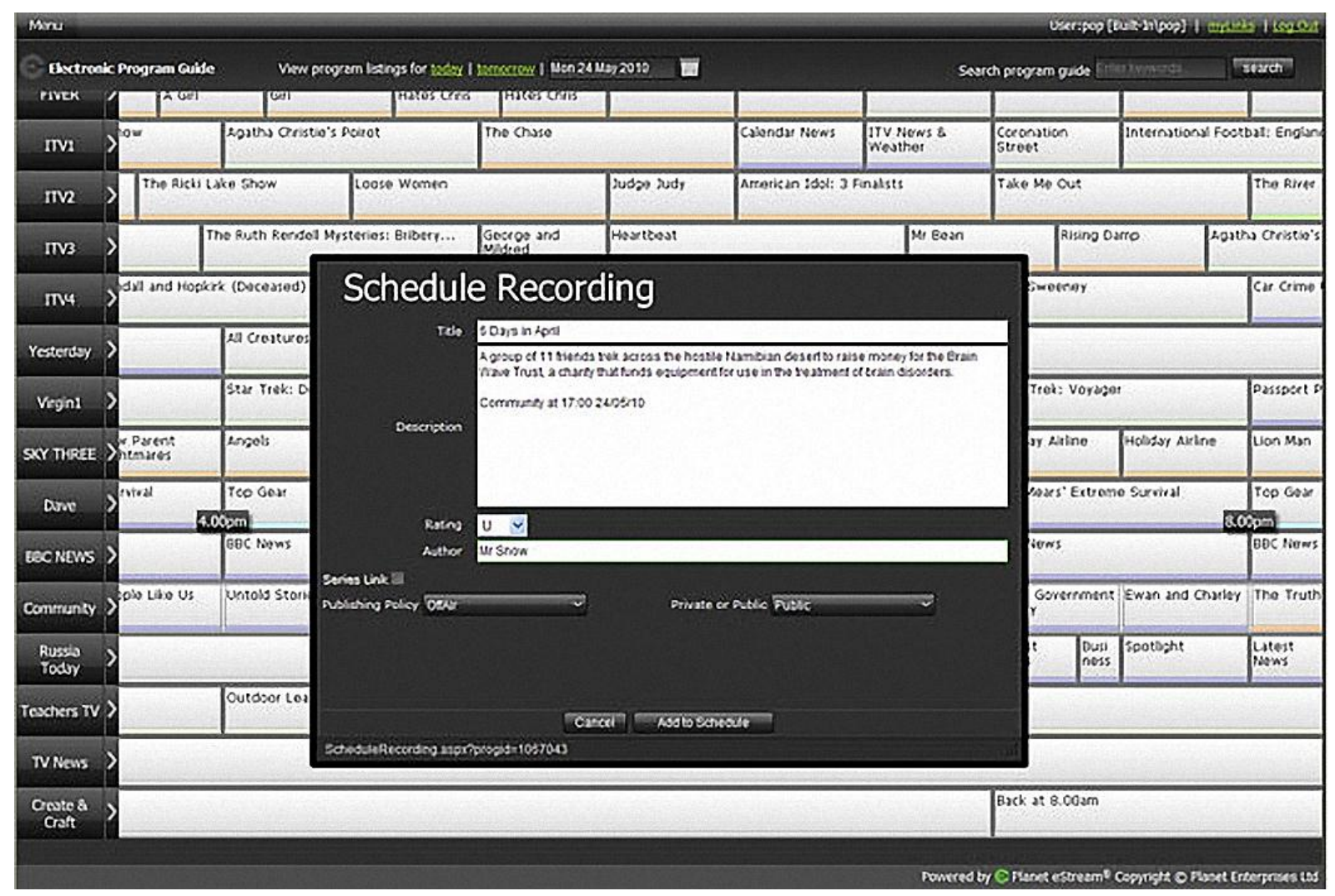

Figure 2.3

"Recording from the EPG with eStream-Reproduced (Planet Enterprises Ltd, 2011)"

The eStream platform is an investment for educational providers. For instance the cost of the Core Solution with the entry level 2TB hardware and the Freeview Module at the time of writing was NZ $\$ 25,000(£ 13,500)$ (Planet Enterprises Ltd, 2011). Nonetheless with the leasing options the company provides more establishments can access this technology which is beneficial for students. Some institutions that use the Planet eStream suite includes the North Tyneside Council, the Spen Valley Sports College, the Bradford College, the Stockton Sixth Form College, the University of Salford, McMillan Academy, the University of Strathclyde in Glasgow, and many others (Planet Enterprises Ltd, 2008).

These institutions can record broadcasted terrestrial television since most of them are covered under the Great Britain's Educational Recording Agency (ERA) which ensures that all the content will be accessible by registered students from their campus for educational purposes only (Educational Recording Agency, 2011). 
Comparing these systems with Replay Me!, existing enterprise products that can record multiple channels simultaneously and index the resulting recordings into a video database already exist, however they are created to solve very specific business issues. They also have very different requirements in terms of hardware resources, which mean that high-end state of the art systems are used in clusters in order to accelerate processes expands the functional capabilities on offer. In comparison Replay Me! needs to be accessible, relatively small and noise free to be accepted into a home environment.

In fact, in RM we are looking to use a standard desktop computer yet still be able to accomplish most of the tasks that enterprises solutions perform. It has also been designed to be very flexible to adapt to the many different configurations of home television set ups, such as native compatibility with satellite and terrestrial television broadcasts. 


\subsubsection{Products aiming at the residential market}

\section{Digital video recorder software (DVR)}

There is a large number of television recording software solutions-also known as personal video recorders (PVR) - available on the market, both commercial and open source. They allow people to watch live television and record any show with a click of a button using the information from an EPG source to automatically pre-set recording schedules. Nonetheless users still need to remember to record shows beforehand in order to watch them at a more convenient time. The most used commercial software is Microsoft Windows Media Center (WMC) which is now included as standard with their operating systems since Windows Vista. According to the official Microsoft Windows Vista website, Windows Media Center can record the shows selected by the user and to allow later playback by listing all the recorded transmissions sorted by date or by title (Microsoft Corporation, 2009). At time of writing the version of WMC shipped with Windows 7 cannot record multiple digital channels at once using a single television tuner (HDTVTunerinfo.com, 2010; Microsoft Corporation, 2011). For this reason it was not considered a viable solution for the stated aims of this project.

\section{Television place-shifters}

One residential solution to the video streaming problem is called Slingbox. This is a settop box that is designed to stream a live video source such as a Sky Television satellite receiver or a DVD player to a computer in another room using the local network in very high quality or even anywhere in the world using the Internet in medium quality. On the remote side, the SlingPlayer software communicates with the Slingbox in order to display the live video stream and a virtual remote control on the screen allows the user to change the channel exactly as if the user was at in front of the source receiver box. This is an answer to the remote viewing issue but unfortunately it is impossible to record anything with this system. As a result, the viewer needs to be available when the show is aired (regardless of the time zone they might be in) to be able to watch it. Moreover this system is designed to stream only one channel at a time to a single seatmultiple viewers wanting to watch the same program from different locations is not allowed. 


\section{Video on demand services}

In comparison, Video On Demand (VOD) websites such as TVNZ On Demand or TV3 On Demand services, offer a categorised set of videos with a text description, but they are usually in medium to low quality and even so they are quite large to download. Consequently this significantly increases costs of internet usage for a residential customer who does not benefit from true unlimited broadband service at a reasonable flat price. Having access to a video on demand service however, is still a real advantage: people can watch what they want at the time they want. Unfortunately not every TV channel offers a VOD service, as the infrastructure required for large scale video streaming over the Internet is a significant investment for broadcasting companies.

\section{"Smart" personal video recorders}

Yet another approach to enriching a viewer's TV experience through "smart" PVR appliances such as TiVo, MySKY HDi, Magic TV and similar.

TiVo-representative of this approach-is a hardware based solution that works in a similar fashion as a DVR, but also includes additional functionalities that enhance the user experience. In fact this system incorporates a feature called "Season Pass" (TiVo) or "Series Link" (My SKY HDi)-as pictured in Figure 2.4-which is designed to automatically record all episodes of a show each time it is aired. TiVo also features "thumbs up" and "thumbs down" buttons on its remote. This is to allow the user to rate television shows. These ratings will then be used by the system to suggest other programs the user might like. (TiVo, International TiVo DVR \& TiVo service, 2009)

According to the TiVo user manual, this system has a grouping option in the playback menu that will organise similar shows in folders helping the user when browsing a large set of recordings. (TiVo, TiVo HD and TiVo HD XL Viewer's Guide - Chapter 1, 2009)

However, TiVo requires a broadband connection to the Internet or to a phone line at all times (Paula Wilson, 2005) in order to operate. Also, this system has not been designed to work with satellite television which is sometimes the only broadcast media accessible 
by rural customers (Hybrid Television Services (ANZ) Pty Limited, 2011). An impediment in terms of basing a solution around TiVo for this project is that shows recorded on TiVo are not directly accessible from the computers of a home network. Digital manipulation is required beforehand. "Tivo Desktop for PC" software needs to be installed to transfer the recordings in a proprietary format onto the computer. To convert the recordings into a format compatible with a mobile device, such as the iPod, the advanced version of the software called "Tivo Desktop Plus for PC" can be purchased for an additional fee. It is important to note that in either case only Windows and Macintosh based software are available from TiVo. (TiVo, International TiVo DVR \& TiVo service, 2009)

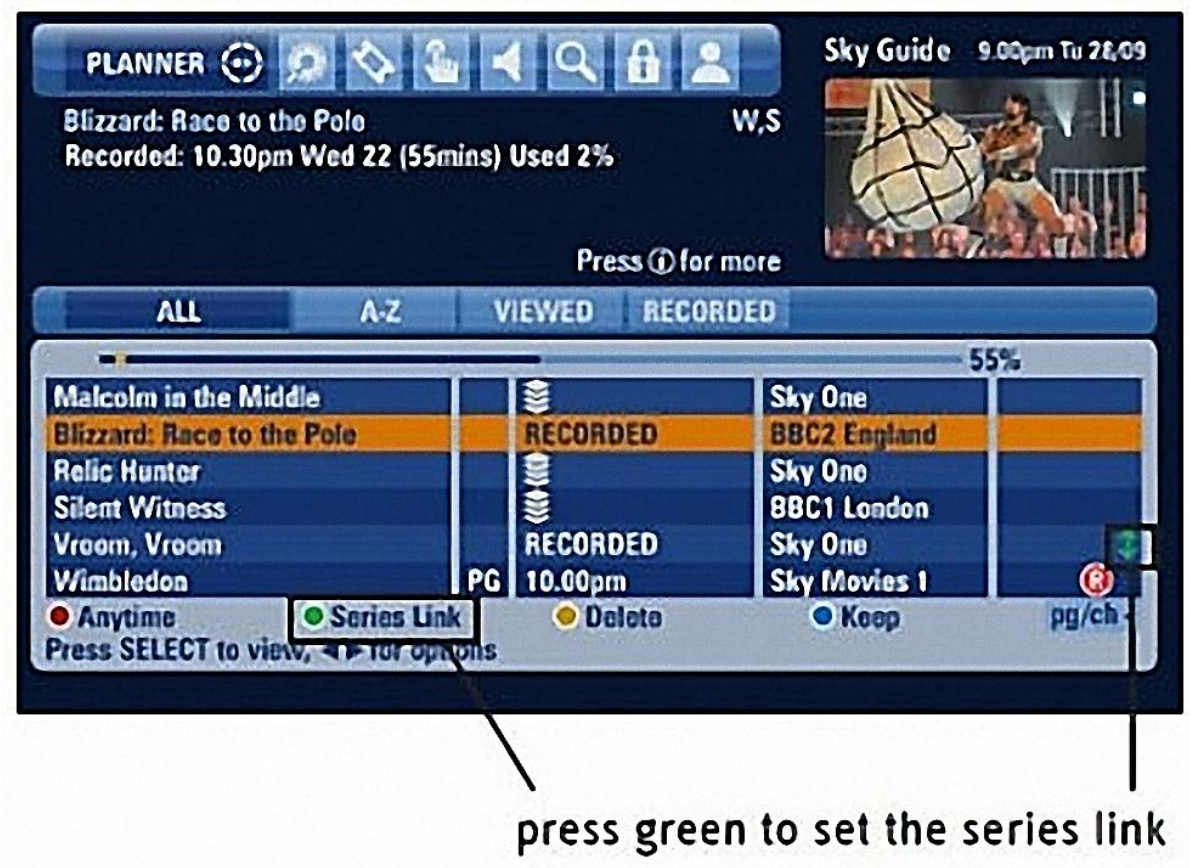

Figure 2.4

“Series Link feature from SKY's PVR—Reproduced (Sky, 2011)” 


\section{Discussion: Comparison with Replay Me!}

Personal video recorders available on the market such as TiVo, MySKY HDi or Windows Media Center (WMC) are capable of recording television. Unlike these PVRs solutions, Replay Me! avoids the need to explicitly initiate the recording, such as selecting which television shows to record (WMC) or a "Season Pass" (TiVo). To achieve this, RM records everything from all the channels by default for a certain amount of time (depending on the disk space dedicated to the system and the number of channels selected). Users do not have to worry about the next show to record, but instead can concentrate on the television programs they would like to watch or archive.

PVRs such as TiVo or MySKY HDi are able to store and organise video files, but they do not allow users to access the content directly from any computer, without requiring to manually transfer the desired file (when this is allowed) beforehand. In comparison, Replay Me! allows all the recordings to be directly accessible simultaneously in streaming form from any computer connected to the home network, from a web browser on any platform (Windows, Macintosh, Linux, etc.) without requiring any special manipulation to transfer the videos in advance. In fact the video player is embedded inside the web pages. Therefore each member of a family is able to watch their favourite show on their personal computer at the same time without having to store anything on their machine. 


\subsection{Existing video digital libraries research projects}

We now shift our attention to consider video content in a digital library context. Handling this medium represents several challenges to librarians, archivist and other curators of content. Due to the size of files involved; due to its temporal dimension; but most of all due to the absence of accurate bibliographic methods that can deal with nontextual attributes (Marchionini \& Geisler, 2002). Furthermore, there is an increasing volume of digitized video content categorised by the content creators (such as TV companies and educational or government institutions), who are not as well-versed in bibliographic mark-up. In addition to this, a huge amount is created by individuals with the generalisation of cheap digital video recording devices that are now embedded in everyday objects such as mobile phones. Together they bring new challenges to curators that were-up to this point-traditionally indexing images and video collections with metadata that was very similar to the one used for text works (Fidel, 1997; Rasmussen, 1997). The need for human-assisted metadata for film archiving was emphasized by Enser and Sandom, for example, whilst at the same time drawing attention to the limitations of automated context-based indexing (Enser \& Sandom, 2002).

Nevertheless, due to this increasing volume of video content and the growing number of users, a considerable effort from the digital libraries communities has been undertaken in order to find practical (and often novel) ways of working with digital video content, the most prominent of which are now presented: Informedia Digital Video Library and the Open Video Digital Library. 


\subsubsection{The Open Video Digital Library (OVDL)}

The Open Video Digital Library is a project that was founded in 1996, with the objective to create a powerful system that can be used by researchers, educational institutions in general and the public leveraging all the progress made in the fields of information retrieval and digital library engineering (Marchionini \& Geisler, 2002).

This main objective, the authors argue, can be reached using several techniques. The first goal the Open Digital Library set themselves was to instantiate and evaluate Marchionini's concept of a "Sharium" (Marchionini G. ,1999). The idea is to directly involve people's participation in order to leverage human time (resource and effort) to contribute to the digital library. The Internet plays an important part in such a model since it allows patrons to easily contribute to the digital library. This is similar to the concept of the Linux archive case study also called by Jones a "contributor-run" digital library (Jones, 2001) or more recently with Youtube. In OVDL this direction was explored through the creation of the Open Video Special Collections web pages, which are designed to study and facilitate the involvement of patrons in DLs (Geisler, et al., 2002).

Another significant aspect of this project was to study the browsing and searching behaviours in electronic environments. In fact digital libraries have their searching and browsing features in the same interface that is used for viewing the content, which led in to new information seeking strategies for their users (Marchionini G., 1995); in fact the authors founded that video is a medium that provides even more opportunities to study these new user behaviours.

User interfaces are the basis of the communication between users and the digital library, so they play a central part in their success. Therefore the OVDL project also focused on evaluating the different possible approaches for DL interfaces by using what Marchionini et al. call "AgileViews" which is a framework specially developed for this purpose (Marchionini, et al., 2000). It is built upon the concept of dynamic query (Shneiderman, 1994) and makes use of interactive graphical interfaces (Card, et al., 1991). 
With these intentions, the OVDL project entered into in a partnership with the Discovery Channel with a project oriented for middle school science and social studies teachers (Marchionini, et al., 1997). Using this context, they ran their first usability experiment with teachers from Baltimore, aimed at evaluating the efficiency of the different video surrogate implementations (Ding, et al., 1999; Komlodi \& Marchionini, 1998; Tse, et al., 1998).

In 1999 the project scaled up with the introduction of a new framework for developing a digital video repository (Slaughter, et al., 2000). From this point on, OVDL received contributions from the U.S. National Archives, the National Aeronautics and Space Association (NASA), the Association for Computing Machinery (ACM), the Carnegie Mellon's Informedia Project, the Howard Hughes Medical Institute, the Prelinger Archives, the Internet Archive and many other reputable sources. By mid-2008, the project referenced more than 4000 non-copyrighted video segments in MPEG-1, MPEG2, MPEG-4, Quicktime and RealPlayer file formats served to more than 40,000 unique visitors each month (Marchionini G., 2004; (Marcos, et al., 2008).

As pictured in Figure 2.5, the central architecture of the Open Video server is based on a Linux machine running an Apache web server. The metadata is stored in MySQL databases and the "AgileViews" user interface is a PHP driven application (Marchionini G., 2006). Due to their very large file size, video files are hosted separately on Internet2 Distributed Storage Initiative Network (Marchionini G., 2004). Newly contributed videos are processed within the technical services of a multistage production system. This is where video recording are digitized, segmented, associated video surrogates (a storyboard, a fast-forward and a 7-second excerpt) are generated and key frames with appropriate metadata are created. The idea was to try to find a balance between file size and a convenient number of segments for a single video title. For this task, the video was digitized (if required) and manually segmented primarily by using Apple Final Cut Pro (Marchionini G., 2006). Since the metadata and video surrogates are an equally important piece of information to the system, they require time and efforts to be created, as part of this process is manual. Actually, metadata was initially enriched using spread-sheet templates, but later on a dedicated cataloguing interface was experimented with (Yang, et al., 2003). Nevertheless, according to Marchionini, due to the time and effort required by this production stage, they have "no immediate solution other than 
continuing to involve our students in the day-to-day operations of digitizing, creating surrogates and metadata, and maintaining the database" (Marchionini G., 2006).

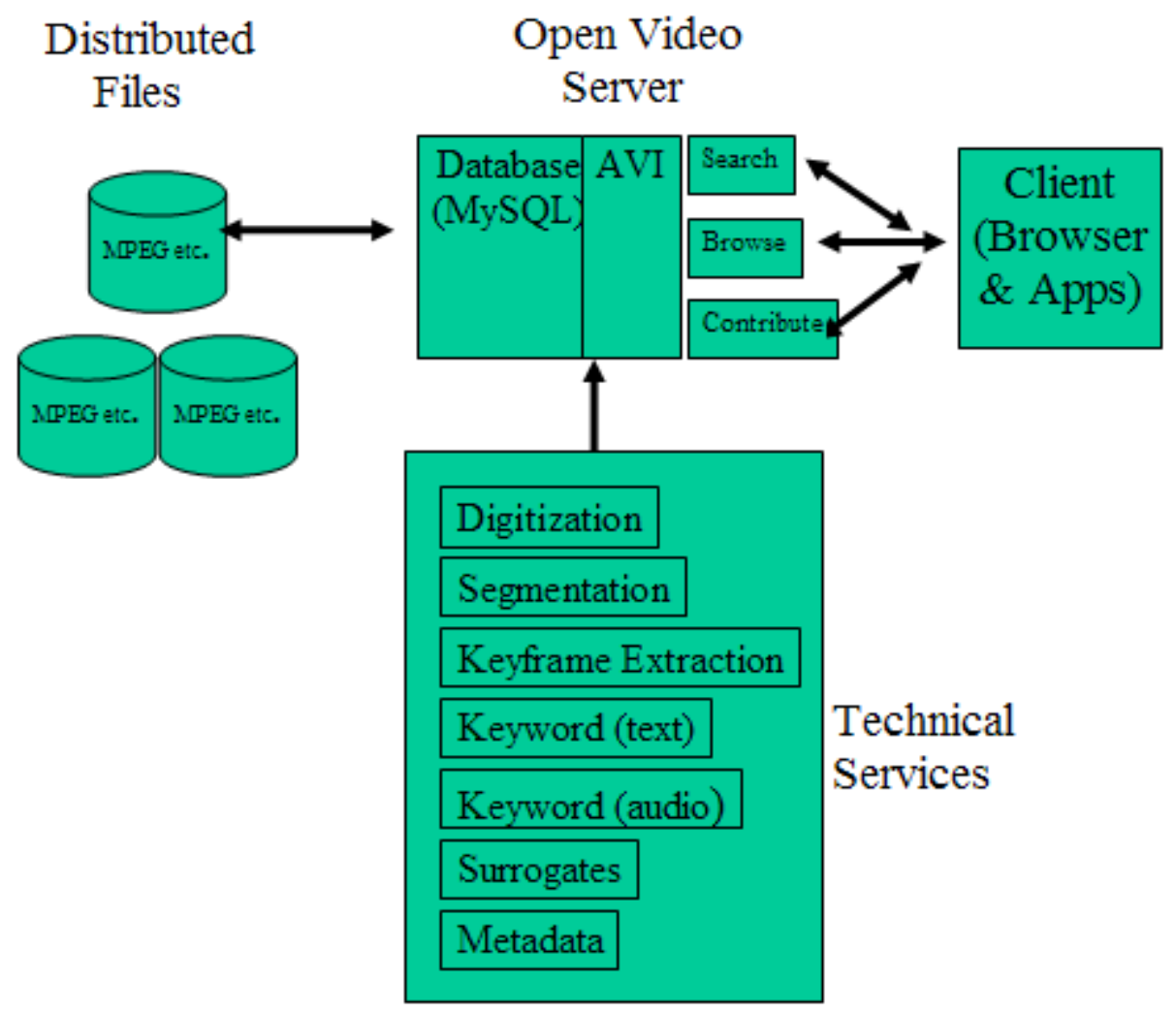

Figure 2.5

"Open Video architecture"-Reproduced (Marchionini \& Geisler, The Open Video Digital Library, 2002) 


\section{Discussion: Comparison with Replay Me!}

In comparing RM with OVDL, a key difference is the target audience: RM is targeting home users whereas the OVDL project targets mainly educational institutions and the wider public, therefore the number of simultaneous visitors could be significant for the OVDL platform while RM only serves members of a family within a home environment. This leads to very different computer requirements: RM must be designed to run on a single residential desktop computer. The OVDL project in comparison is a heavy weight contender-it leverages the power and storage of distributed servers and a professional production set with enterprise grade tools for processing the new videos. Although the OVDL makes use of clever video surrogates with undeniable benefits for the users seeking video content, it does not have a real time requirement and the generation of these interesting metadata enriched features are still a lengthy and manual process for some parts. On the other hand, RM needs to cope with a continual flow of new recordings from multiple TV channels and needs to be able to process them in real time to avoid an exponentially growing queue of recordings waiting to be processed. As a result only the minimum of processing for each video file can be afforded in RM ideally using the most efficient methods available, without requiring any human intervention at any stage. Nonetheless, with the rapid increase in performance of residential computers, one can easily imagine that such advanced video surrogate extraction techniques-such as employed in OVDL-will become possible in real time in the not too distant future even on multi-gigabyte files such as the recordings made from high definition broadcastings. 


\subsubsection{Informedia Digital Video Library}

One of the most prominent and comprehensive research projects that specifically focus on video digital library potential is the Informedia Digital Video Library (IDVL) project. It uses state of the art technologies in order to create full-content search and retrieval digital video libraries (Christel, et al., 1995). The project was initiated in 1994 at Carnegie Mellon University, funded by the US National Science Foundation, the US Defence Advanced Research Projects Agency (DARPA) and the National Aeronautics and Space Administration (NASA) under the US Digital Library Initiative (Wactlar, et al., 1999). In total this project has received over $\$ 8$ million in funding from government sponsors and from large corporations such as Intel, Microsoft, Motorola, and others (Foley, 1997). Informedia Digital Video Library distinguishes itself from others by using automated intelligent mechanisms to extract information from videos (Christel, et al., 1995).

The main goal of the IDVL project is "to enable full content search from digital video libraries" (Christel, et al., 1996; Wactlar H. et al., 1996). The approach taken was to make use of speech recognition, image and natural language processing (Christel \& Martin, 1998) Expressed with a user-centric views, this project aims to let library users search the video medium the same way they are used to searching text content.

To enable this goal, Informedia has incorporated a locally developed open source speech recognition software-called Sphinx-to allow the automatic transcriptions of videos such as broadcast news stories. Powerful image processing algorithms were employed to detect scene boundaries, to recognize faces and to allow image similarity comparisons. Even the text that is embedded in the video can be recognised and becomes searchable through the use of Video Optical Character Recognition (VOCR) techniques (Wactlarv, et al., 2000).

As described by the authors (and pictured in Figure 2.6), newly captured videos are:

- segmented into smaller pieces;

- analysed in order to extract metadata and other alternative video representations; and

- indexed in a system that allows fast information searching and retrieval. 


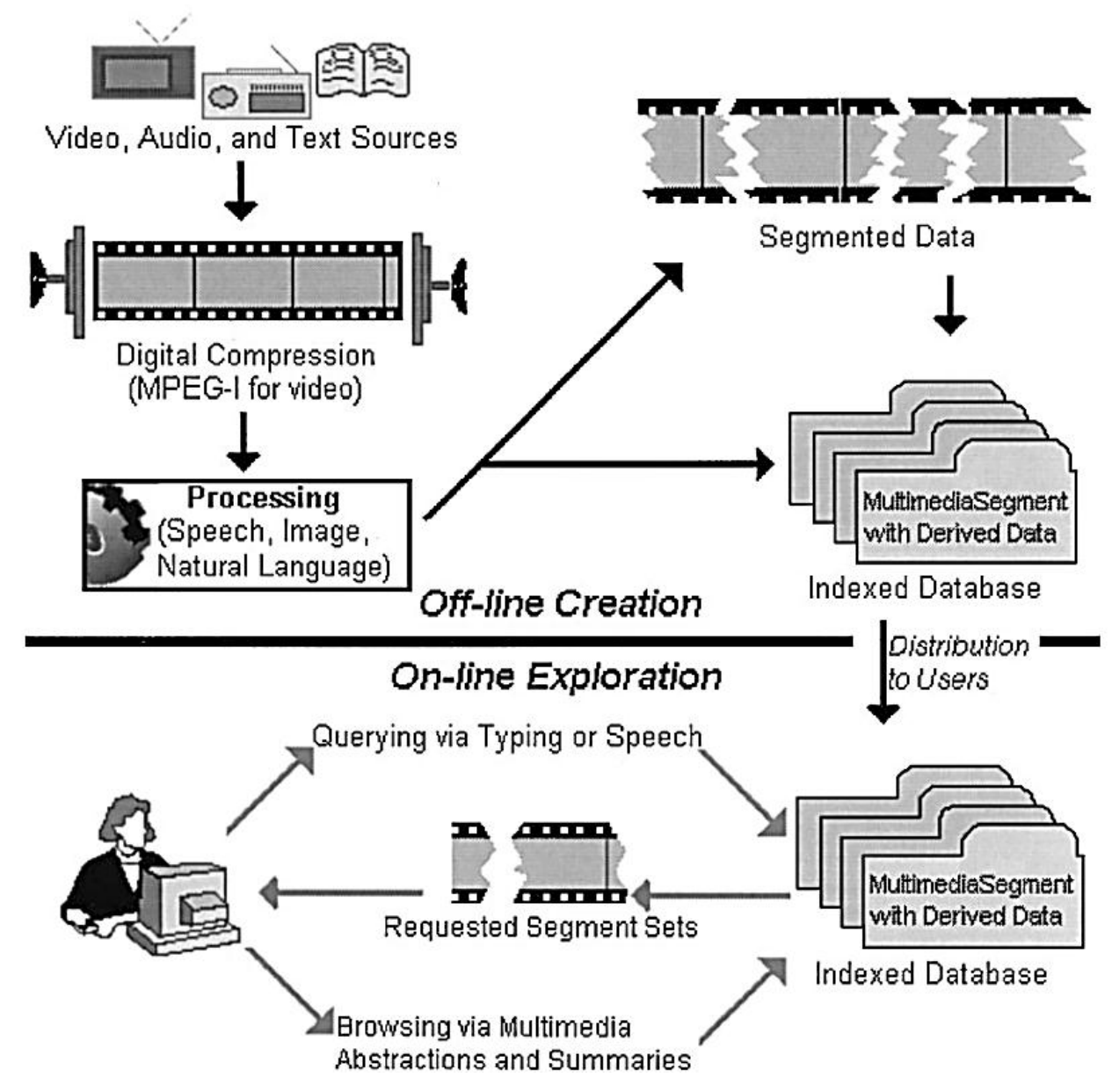

Figure 2.6

“Informedia Digital Video Library System overview"-Reproduced

(Christel \& Martin, 1998)

The IVDL project has a special collection called "News on Demand" which is similar in some ways to RM. This is a library of captured television and radio news continuously recorded from a satellite feed on a daily basis. This particular collection's goal was to develop a system that is totally automated (Witbrock \& Hauptmann, 1998). By October 1999, the system had made available about 1.5TB of news video content which included 
more than 1600 new broadcasts or the equivalent of 40,000 news stories dating back to 1996 (Wactlarv, et al., 2000).

One of the main aspects of this video collection is the use of speech recognition to generate the transcripts for the news stories. This process was done in two phases: first a quick pass of the Sphinx-II recogniser with a small vocabulary set was applied then a second pass with Sphinx-III with a larger vocabulary set is used lower the error rate. The subtitles extracted from the shows, when present, are also used to improve the precision of the resulting transcript (Jang \& Hauptmann, 1999). Nevertheless, this process takes time (100x real time for high precision) and the end result is error prone-for example, some English accents could not always be recognised properly-however even with this error rate, in practice it was found to be accurate enough to allow searching inside video content (Wactlarv, et al., 2000; Kukulska-Hulme, et al., 1999).

Image processing is also applied to segment the videos into smaller shots, to create an index for supporting the image similarity feature but also to generate metadata from graphical elements. The technology used can detect significant colour shifts and advanced optical flow characteristics — such as camera panning and object motion-in order to ensure that each created shot contains an entire coherent sequence (Zhang, et al., 1995).

The Informedia Digital Video Library is also capable of associating names with faces occurring in the captured news videos. A commercial face detection and tracking software has been used to find faces, then video optical character recognition (VOCR) technology is used to extract the names overlaid on the screen and face similarity algorithms used for information retrieval (Kubala, et al., 1998). The VOCR technique involves the following three steps (Wactlar, et al., 1999):

- frames that are likely to contain text are first identified;

- regions of these frames that contains clustered sharp edges (text) are postprocessed using consecutives frames to improve the text optical quality; and

- the text is then extracted using commercial OCR software.

In addition, the "News On Demand" collection identifies the locations of the news stories as they get extracted from the generated transcripts. These possible locations are then cross-referenced against an inventory list of possible country names from around the 
world to determine their coordinates in order to place them on a map (Environmental Systems Research Institute, Inc., 1999).

\section{Discussion: Comparison with Replay Me!}

The Informedia Digital Video Library is an impressive project that has demonstrated that "previously inaccessible data can be derived automatically and used to describe and index video segments" (Wactlar, et al., 1999). However, unlike RM, their project was not require to operate in real time. In their design this permitted them to use large vocabulary sets for voice recognition and very computationally intensive algorithms for video processing (Wactlar, Kanade, \& Smith, 1996).

The Informedia Digital Video Library project is similar to RM in the fact that they are both designed to operate fully automatically. In the "News On Demand" collection, only the news stories from Cable News Network (CNN) were recorded (Wactlar, et al., 1999). This left sufficient time between each recording to allow the application of several computational intensive video and audio processing techniques, and even here some of the operations were parallelized on several machines to decrease the time required for processing (Wactlar, et al., 1999). The Replay Me! project in comparison, has different requirements as it should be able to operate considerably faster than real time in order to process all the newly recorded TV shows on several channels simultaneously on a single desktop computer. Still, it is irrefutable that the results from state of the art processing used by IVDL to get enriched metadata are a true advantage when it comes to searching within videos. We return to this point and discuss it further in the conclusion of this thesis (Chapter 5). 


\subsection{Missing parts}

In this section we pose (and answers) the question: can the envisioned home-based TV system be provided by existing work? If not, then what parts are missing?

Each of the enterprise products reviewed earlier were designed for solving very specific niche applications and therefore are not very useful in general to home users. In addition, these solutions are expensive and are largely inappropriate for home environments due to the amount of noise they produce and/or the space required.

The digital video library research projects reviewed have pushed the boundaries of video processing by making content within videos searchable that was not accessible before. These academic projects are designed with video archiving in mind and for distribution to a large audience via the Internet. Therefore they use distributed systems for the archiving of and the processing of the recordings. Complex and time consuming algorithms are executed in order to get more interesting information from videos since these systems do not require real time operation. Also, as explained earlier, the Open Video Digital Library requires some operations to be carried out manually during the importing phase of new video recordings.

These are very different requirements from Replay Me!, as our project aims to cope with a continual flow of new recordings from multiple television channels in real time using a single desktop computer without any human intervention.

Commercial products targeting the residential market intentionally restrict some functionality. Motivated, for the most part, to ensure the company can maintain its revenue, they nonetheless mean the products provided do not match up to the requirements for Replay Me!.

Examples of these intentional restrictions are:

- some products do not have streaming capabilities even within the user's house (TiVo, MySKY HDi),

- others can stream but only to a single seat and do not offer recording facilities (Slingbox),

- some can only handle terrestrial television (TiVo),

- or only satellite TV (MySKY HDi), 
- most can only record one channel per tuner (at time of writing: TiVo, MySKY HDi, Windows Media Center),

- some do not allow the user to extend the storage capability through an external disk (MySKY HDi),

- some require an Internet at all times for operation (TiVo).

Moreover these products have a cost which is sometime comparable to the price of a home theatre computer and in some cases even after the full payment of the appliance the user never owns the hardware. In fact if a client of MySKY HDi decides to end their subscription, the box must be returned to SKY (Tech Talks, 2010). Unlike some previous approaches, RM aims for a low cost since it is using only open source software and a single home desktop computer with digital television capturing capabilities. 


\section{Broadcast Television}

In this chapter the terminology and the challenges involved in the technology used by digital broadcast television are first explained, then implementation details on how Replay Me! processes the constant input of live digital TV broadcasts are provided.

\subsection{Compression}

During video digitalization, the resulting uncompressed video takes a huge volume of space, which is particularly true for the increasing definition of the content created today. This makes digital video difficult to store and transmit. As a result, video is compressed using CODECs (compression/decompression programs also known as coder/decoder) in order to reduce their size.

There are two main forms of data compression:

- Lossy compression;

- Lossless compression.

With lossless compression, when the data is compressed and then decompressed it is exactly identical to the original. The data is arranged in a way that is more efficient, to save some space but all the information is still available, nothing is lost. On the other hand, lossy compression allows more space savings than lossless compression but at the price of discarding some of the information detail from the original file.

In terms of television broadcasting compression, the idea of using lossy codecs is to greatly reduce the volume of data to be transmitted, in a way that the discarded information from original videos is not obvious to the naked eye of most viewers. If lossy compression is pushed too far, the process becomes so destructive that unwanted image distortions become obvious to viewers-these side effects of over-compression are called "compression artifacts" (Amerasinghe, 2009).

Video is made of a series of still images - also called frames - that are displayed rapidly one after another to give the illusion of moving images. To the human eye, the illusion of motion becomes convincing enough from around 24 frames per second (fps)-which is 
the cinematic frame rate. In Europe, Australia, parts of Asia and New Zealand the PAL ${ }^{2}$ standard uses $25 \mathrm{fps}$ while in the U.S. and Japan the NTSC ${ }^{3}$ format consist of $29.97 \mathrm{fps}$.

The fact that a video is a series of images is important to better understand how video CODECs work. In reality there are two fundamental ways of compressing video content:

- Compressing individual images by themselves;

- Compressing a series of images together.

Compressing individual images by themselves-also known as intra-frame compression-means that you only need to run a compression algorithm on each original input frame and save the resulting data to the output file. This process ignores what is happening between multiple frames in a sequence; as a result a large amount of redundant information is stored given the similarity of adjacent frames. In the case of video broadcasting, this means that very similar images are sent over and over again, resulting in a waste of the bandwidth available (Honovich, 2008).

Recent CODECs such as H.264-also known as MPEG-4 Part 10/AVC for Advanced Video Coding-are designed to exploit this inter-frame similarity as well as intra-frame compression to further reduce or remove the information to be transmitted (Axis Communications, 2008). These CODECs are still sending whole images (also called Iframes or intra frames) at regular intervals, however the rest of the time only the updates describing the changes between frames are sent-this is known as inter-frame compression. Since most videos contain frames with many parts that remain the same, this type of compression results in very significant savings of disk space or bandwidth. Alternatively from a different perspective, this means that a higher video quality can be achieved for a same bit rate. Nonetheless the biggest downside of this standard is the processing power required to "reconstruct" the final video during decompression, as many complex operations that consume large amounts of CPU cycles are involved (Honovich, 2008).

H.264 has seven profiles which are designed to describe the set of features that can be used by the encoder in order to limit the decoder complexity depending on the target audience. Depending on the profile used, the encoder may use different types of

\footnotetext{
2 PAL or Phase Alternating Line.

${ }^{3}$ NTSC or National Television System Committee.
} 
frames-pictured in Figure 3.1-such as I-frames, P-frames and B-frames (Axis Communications, 2008).

- I-frames (or intra-frames) are completely self-contained and can be decoded without reference to any other frames. They do not generate many artifacts but they consume more space. They are required for random access within a video stream such as fast-forward, rewind, etc. I-frames also serve as resynchronisation points for viewers if the transmission stream has been damaged during transport.

- IDR-frames (or “Instantaneous Decoding Refresh" frames) are like I-frames but they force the decoder to refresh itself by explaining that from this point on nothing is referring to previous frames. They act as grouping cues.

- P-frames (predictive inter-frames) make reference to parts of an earlier I and/or P frame. They are very sensitive to transmission errors because of their complex dependency to earlier I/P-frames however they require fewer bits than I-frames.

- B-frames (bi-predictive inter-frames) make reference to both an earlier reference frame and a future frame.

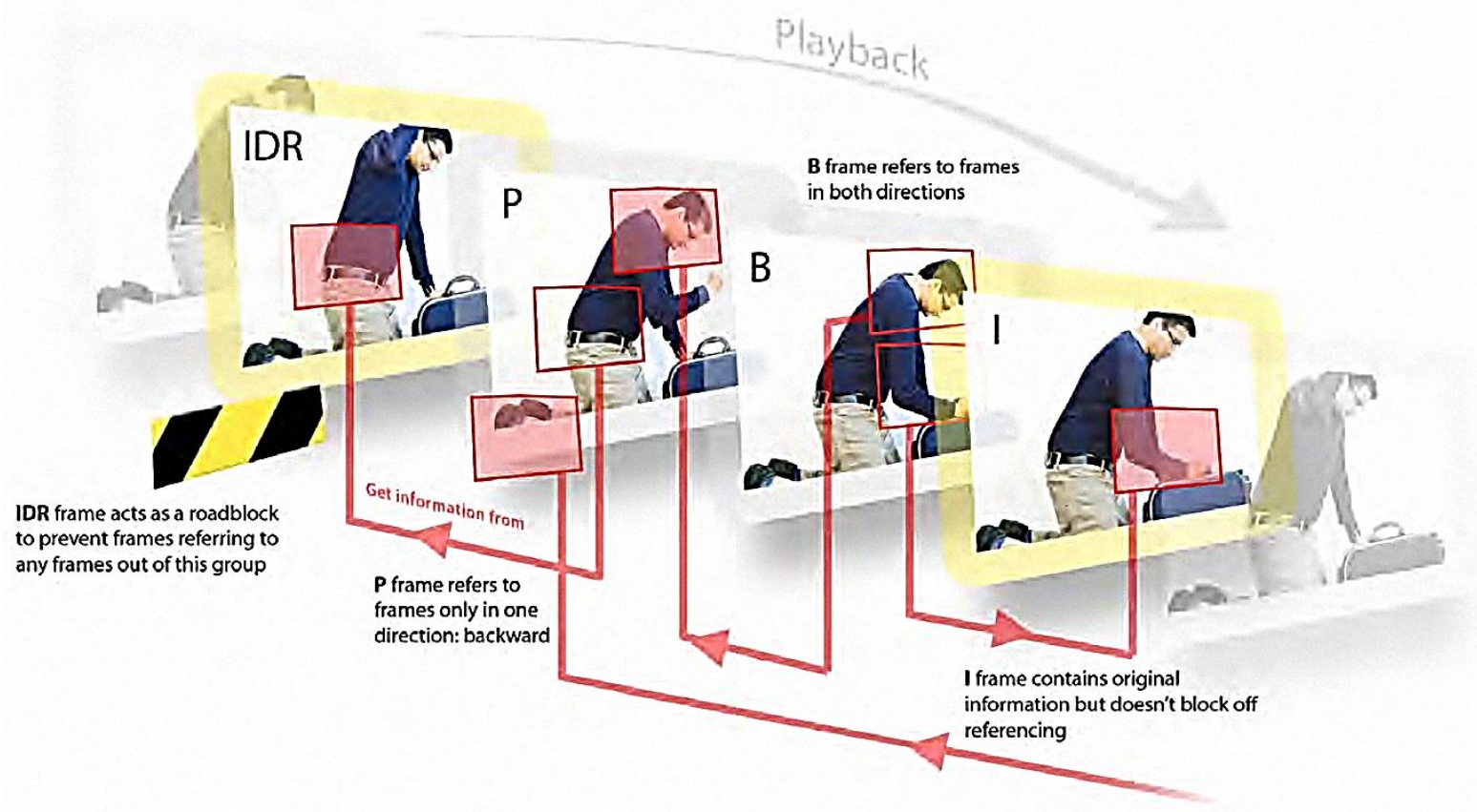

Figure 3.1

"Frame types-Reproduced (Amerasinghe, 2009)" 
As can be seen, decoding a video stream in H.264 for playback is quite a computationally complex operation. It is also a challenge to efficiently and quickly decode frames at regular intervals to create a fast preview because of this issue of frame dependency, but in the case of broadcast television, possible transmission errors during transport also need to be taken into account.

The H.264 standard has been widely adopted in many different areas the web, television broadcasts, Blu-ray disks, etc.) since it is the result of a joint project between standardisation organisations in the telecommunications and IT industries (Axis Communications, 2008). It was created to address weaknesses of practically all previous compression standards, including earlier versions of the MPEG-4 standard itself! For instance, given a fixed video quality, an average bit rate reduction of $50 \%$ is possible with the new standard compared with other video standards, and better recovery schemes are built in for transmissions errors over various networks (Axis Communications, 2008). These improvements over other standards made it the CODEC of choice for efficient television broadcasting. In New Zealand, H.264 been selected for the terrestrial free-to-air television broadcasting platform (Freeview|HD).

Audio also needs to be compressed to achieve maximum broadcasting efficiency. Since New Zealand was a late adopter of digital terrestrial television broadcasting, they have watched what other countries have done in this field and finally decided to use one of the highest performance CODECs-HE-AAC - in regards to quality and efficiency for audio compression. High-Efficiency Advanced Audio Coding (HE-AAC also known as AACplus) is a recent audio compression CODEC developed by Coding Technologies (acquired in 2007 by Dolby Laboratories) optimised for very low bitrate applications, and is defined as an MPEG-4 Audio profile.

In order to let all these different data types coexist together (video, audio, EPG, teletext, subtitles, etc.) the MPEG transport stream (TS) media container is used by Freeview|HD. This container format-specified in MPEG-2 Part1-has been designed for environments that are not always reliable for data transmission, such as terrestrial or satellite broadcast (International Telecommunication Union, 2010). 


\subsection{Transmission}

Most countries are now switching from analogue to digital television broadcasting since it allows a better use of the available radio spectrum, meaning that more services in better quality can be offered using fewer frequencies. In New Zealand, the switch to Digital Terrestrial Television (DTT) started in 2008 and is commercially known as Freeview|HD.

Using the same standard as in Europe, Freeview|HD is broadcasted in DVB-T (Digital Video Broadcasting - Terrestrial) which is a terrestrial transmission system developed by the DVB Project. This new national digital television network was designed and deployed by Kordia with over 18 broadcast sites and 54 new transmitters, and as of August 2011 it is now covering over $86 \%$ of the New Zealand population(Kordia (NZ) Ltd, 2008; Digital Video Broadcasting, 2011.

This network has the capacity for 3 radio frequency (RF) channels or multiplex (MUX):

- TVNZ;

- TVWorks;

- Kordia for regional and niche broadcasters.

In fact, digital TV broadcasting enables multiple channels to be multiplexed together into a single MUX and then broadcast using one frequency. These channels can be of different resolutions (standard definition or high definition) and the bandwidth allowed to each channel is automatically arranged using a statistical multiplexer. The idea is that one multiplex has a given fixed bandwidth (in NZ at time of writing around 26Mbps) but not all channels require the same amount at a given point in time in real time. As pictured in Figure 3.2, channels with fast changing scenes are automatically allocated more bandwidth while other channels with less complex scenes are allocated less bandwidth.

Moreover, with the exceptionally efficient H.264 CODEC used to compress the video, up to 8 channels in standard definition can be broadcasted using a single frequency instead of only one analogue channel (Kordia (NZ) Limited, 2008).

On the receiving side, the digital television tuner "tunes" to a specified radio frequency and gets an entire multiplex. The TV channel selected by the user is extracted from the MUX and displayed on screen. The stream is not always error-free due to transmission 
errors. This can lead to occasional "blank screens" or "artifacts" during playback, especially in areas where the signal is weaker.

Satellite television broadcasting is comparable to DTT since it is using the same principle of multiplex of channels per frequency-also called a transponder.

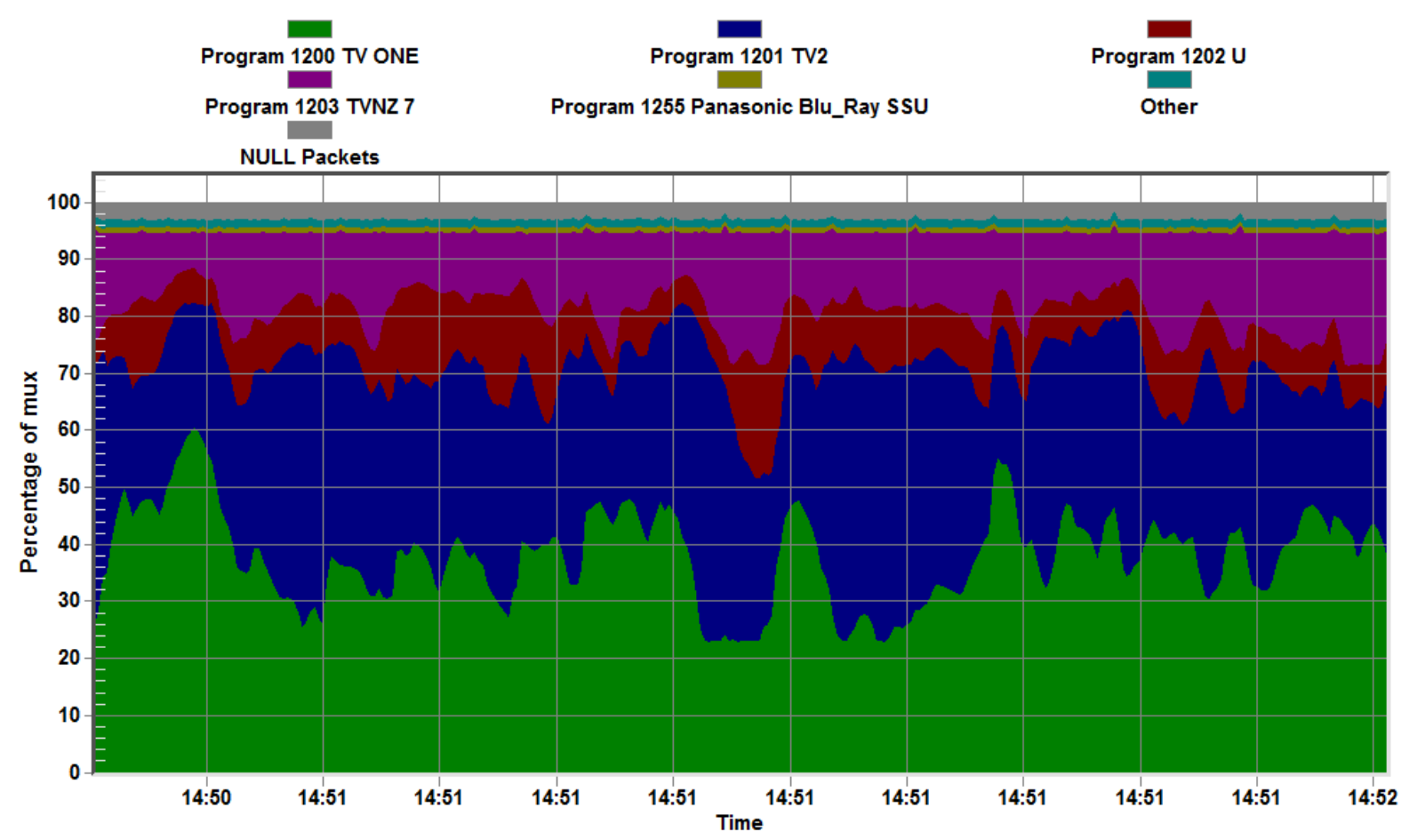

Figure 3.2

"Usage Graph of the TVNZ Multiplex on 16/09/2011" 


\subsection{Processing Input and Output}

In this section the challenges of recording a multitude of live digital channels simultaneously are introduced, along with the solutions that were implemented.

\subsubsection{Multiple channels concurrently}

In a previous project a proof of concept, a video digital library that was only capable of recording one channel at a time with a more limited set of functionality was developed (Roüast, 2010). This was useful to determine the technical requirements for expanding to a project that would be able to record multiple channels simultaneously.

In this proof of concept project, each show recorded was then post-processed to improve the image quality (such as de-interlacing-see section 3.3.2) and then further recompressed using H.264 at a very low bitrate to:

- Lower the decompression complexity to allow playback on some entry level portable devices,

- Allow smooth playback on computer and flat TV screens,

- Add compatibility with Adobe Flash Player for easy video playback in most web browsers,

- Reduce the bandwidth required allowing direct off-site video streaming from a home ADSL connection.

However the results from this previous experiment highlighted the high CPU cost to achieve the required processing. In fact, as pictured in Table 3.1, the proof of concept design was not scalable to be able to practically record multiple channels concurrently. In reality, on our prototype machine the transcoding and filtering of a video in standard definition format is done only 2.4 times faster than real time; and the same process is completed at approximately real time when using a recording in high definition as the input file.

From the principle that, in the vernacular, "disk space is cheap" compared with processing power, it was decided for Replay Me! to import the raw data from the broadcast directly into the video collection, without any recompression or image postprocessing at this point. This have the benefit of preserving every sub-stream associated 
with the video such as the subtitles, multiple audio tracks and most importantly the video quality is kept at its best.

In terms of issues to be faced, due to the technical specifications of the video being directly recorded from the tuner, this required a compatible video player to be found that can be embedded into a web page. This video player should also be able to postprocess the video in order to adapt its format to be displayed on computer and flat TV screens. More details on the implementation of these features are covered in Chapter 4.

\begin{tabular}{|c|c|c|c|c|}
\hline \multirow{2}{*}{} & \multicolumn{2}{|c|}{ Encoding only } & Encoding + De-interlacing filter \\
\cline { 2 - 5 } & Encode duration & Speed $\boldsymbol{\Delta}$ & Encode duration & Speed $\boldsymbol{\Delta}$ \\
\hline $\begin{array}{c}\text { SD sample } \\
\text { Duration: }\end{array}$ & $00: 03: 35$ & $3.3 \mathrm{x}$ real time & $00: 04: 55$ & $2.4 \mathrm{x}$ real time \\
$\mathbf{0 0 : 1 2 : 0 2}$ & & & & \\
\hline $\begin{array}{c}\text { HD sample } \\
\text { Duration: } \\
\mathbf{0 0 : 1 1 : 3 4}\end{array}$ & $00: 07: 30$ & $1.5 x$ real time & $00: 10: 32$ & 1.1 real time \\
\hline
\end{tabular}

“Time elapsed during transcoding and filtering video samples to H.264" 
The fact that digital TV broadcasts are multiplexed plays to the advantage of Replay Me!, as this means only one tuner per frequency is required to record all the shows it carries, as the entire MUX is directly accessible. The software only needs to create sub-transport stream (TS) files for each recorded channel taking the corresponding streams (video, audio, subtitles, etc.) from the main transport stream being aired. This process is not computing intensive as no image processing of any sort is applied to the content; therefore the computer processor is not the limiting factor for recording multiple channels (as in the proof of concept project) as pictured in Figure 3.3. These graphs were generated by measuring the average CPU usage of the process taking care of concordant recording-and de-multiplexing-of multiple TV channels on our prototype system for a period of 15 minutes.

When recording 16 channels simultaneously-which corresponds to all the channels available on Freeview|HD at the time of writing-the CPU of our prototype machine (Intel ${ }^{\circledR}$ Core $i 5^{\mathrm{TM}} 3.33 \mathrm{GHz}$ ) is only used at a $1 / 20^{\text {th }}$ of its total capacity.

\section{CPU usage during the concurrent recording of multiple TV channels on Freview|HD}

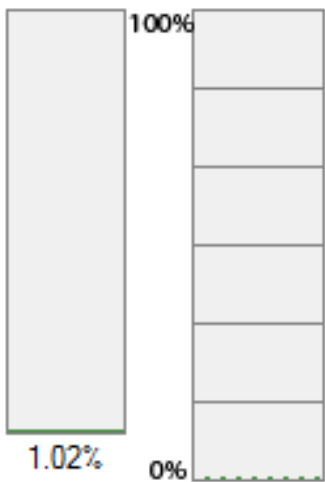

One recording

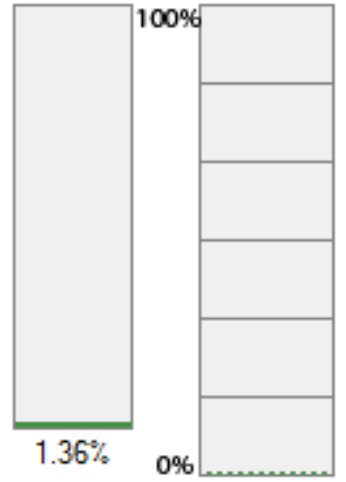

4 recordings

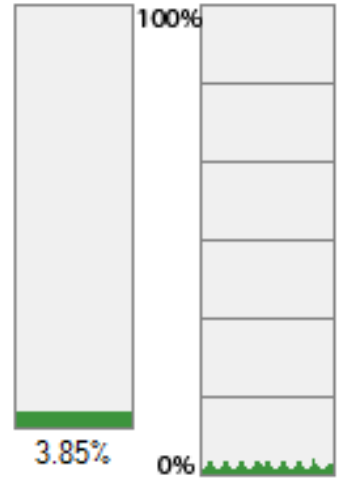

8 recordings

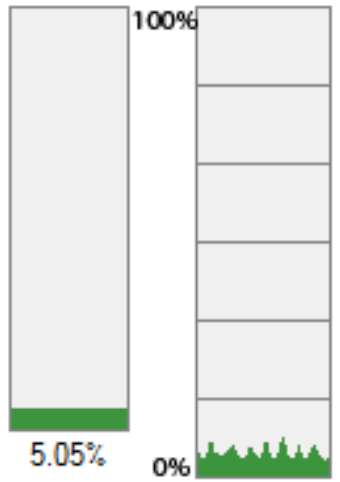

16 recordings

Figure 3.3

"Low CPU usage during concurrent TV channel recording" 
As described in the previous section, 3 multiplexes are used to broadcast all the channels of the Freeview|HD DVB-T platform. The listing of channels per MUX at the time of writing is pictured in Figure 3.4. At a technical level, one tuner per MUX is required to be able to record all the available channels on the New Zealand free-to-air DVB-T platform. However in our implementation one more tuner is required to periodically extract the EPG from the live stream since the other tuners are continuously in use. In the prototype built for the experiment evaluation reported in Chapter 6 (that became known as RM), only two internal dual DVB-T tuners have been used to record everything from Freeview|HD and to extract the 7 day electronic programme guide every 24 hours.

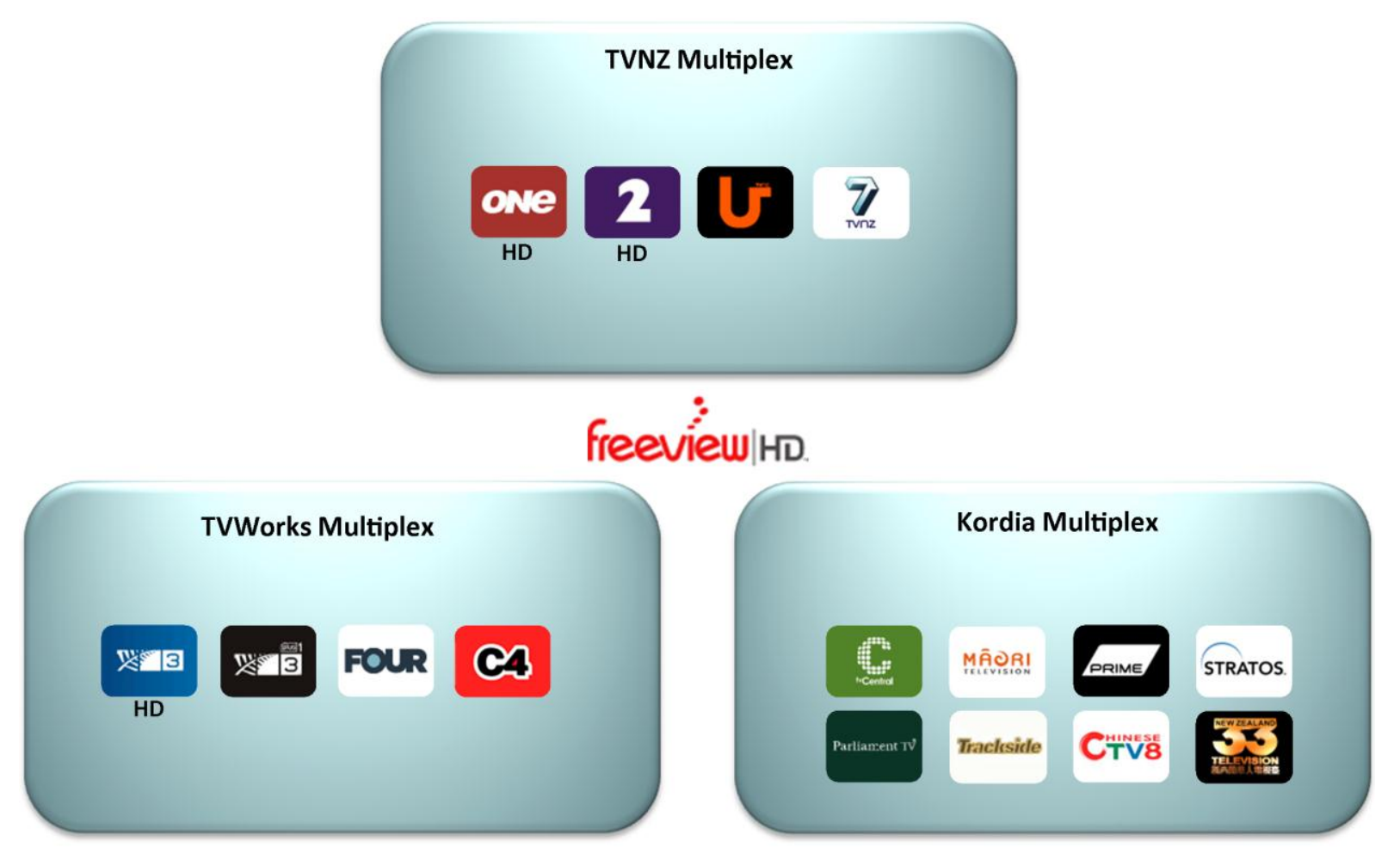

Figure 3.4

"Television channels listing per multiplex on Freeview|HD in 2011" 
Depending on the broadcast medium, not only does the technology for transmission differ but also the compression formats and resolution for video, audio, subtitles-as detailed in Table 3.2 which compares New Zealand Freeview Satellite and Terrestrial platforms. To guarantee maximum compatibility, Replay Me! has been designed to avoid dependency on any particular broadcast formats to be able to provide full functionality. This project uses multiple open source applications for specific activities that have been carefully selected to provide the broadest compatibility possible allowing RM to be applicable in countries using different standards.

\begin{tabular}{|c|l|l|}
\hline \multicolumn{1}{c|}{ Freeview Terrestrial } & \multicolumn{1}{c}{ Freeview Satellite } \\
\hline Transmission & DVB-T & DVB-S \\
3 Multiplexes & 2 Transponders \\
\hline Video & H.264/MPEG-4 AVC & MPEG-2 \\
& Standard definition: 576i & Standard definition: 576i \\
\hline High definition: 1080i & \\
\hline Audio & HE-AAC LATM - Stereo & MPEG-1 Layer2 - Stereo \\
\hline Subtitles & AC3 - Dolby surround & \\
\hline \multirow{2}{*}{ DVB image based format } & Text based format \\
\hline \multirow{2}{*}{ Electronic Program Guide } & MHEG-5 application & MHEG-5 application \\
& EIT EPG now and next only & EIT EPG for all programmes \\
\hline
\end{tabular}

Table 3.2

"Formats comparison between Freeview Terrestrial and Satellite in New Zealand" 


\subsubsection{Real time operation and hardware limits}

Real time processing is critical: every hour there is 60 minutes of content times the number of channels recorded to be imported and indexed into the digital library. Having this volume of data coming down continuously, any process applied to the recordings must be optimised to avoid an increasing quantity of recordings waiting to be ingested into the system. More details on the algorithms applied to the recordings are given in the next chapter. Here we concentrate on where/what the hardware limits are.

This continual stream of data presents our first key hardware issue to consider: how quickly can all this be stripped out of the mux and be written to disk? In fact, the bandwidth available to broadcast the channels on Freeview terrestrial is much larger than on the satellite platform or even on Internet based services. Therefore broadcasters, who usually own their multiplex, try to make use of all the available bandwidth by adding high definition channels to their offering and by compressing the video to the minimum-thus keeping the highest possible quality. This results in multiple recordings that are encoded in high bitrates that needs to be written to disk in real time.

Figure 3.5 illustrates the average bitrate in Mbps used by each channel broadcasted on Freeview|HD, while Figure 3.6 compares the average speed required to record all channels ( 3 in high definition, 13 in standard definition) with the maximum average sequential writing speed in $\mathrm{MB} / \mathrm{s}$ of a single hard disk drive running at $7200 \mathrm{rpm}$ (rotation per minutes) that is used in our prototype system. The first graph shows that, as expected, most of the bandwidth is dedicated to the video while the audio and subtitles takes only a small proportion of data per channel. Moreover we can also see that one high definition channel uses about the same bandwidth as 3 standard definition channels. The later graph was generated by using a benchmark tool to calculate the maximum writing speed of the HDD (hard disk drive) used for our experiment in the best case scenario (sequential writing without any simultaneous reading).

The disk I/O (input/output) usage of the process taking care of the recording was also measured to avoid any extra I/O generated by the operating system. While it is clear that this hard disk can certainly handle 16 concurrent recordings, these measures are given for the best case scenario. In fact, the HDD also needs to read data the same time to serve the digital library users, and this can affect its writing capacity. 

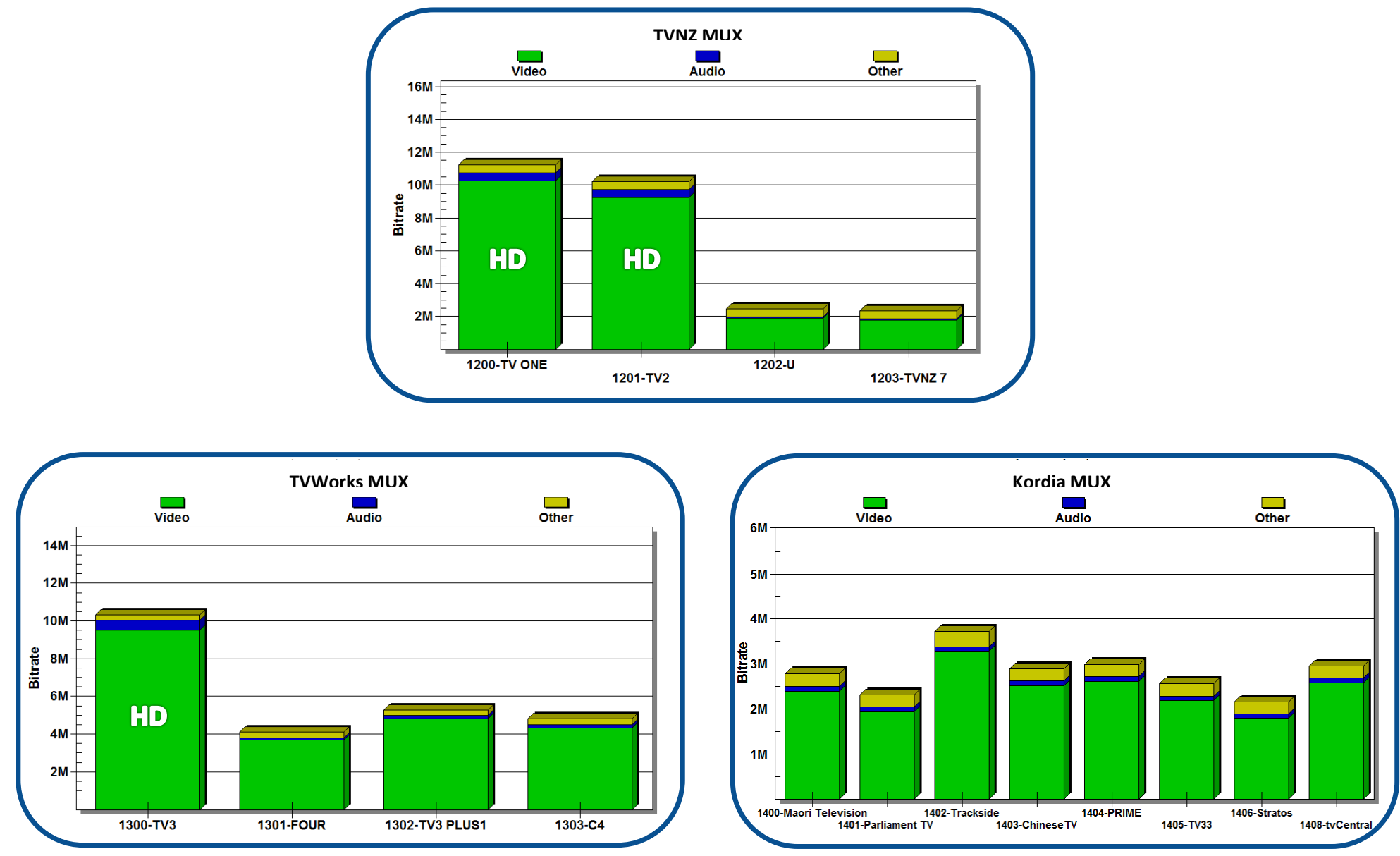

Figure 3.5

"Bandwidth utilisation in Mbps per TV channel on each Freeview|HD multiplex" 


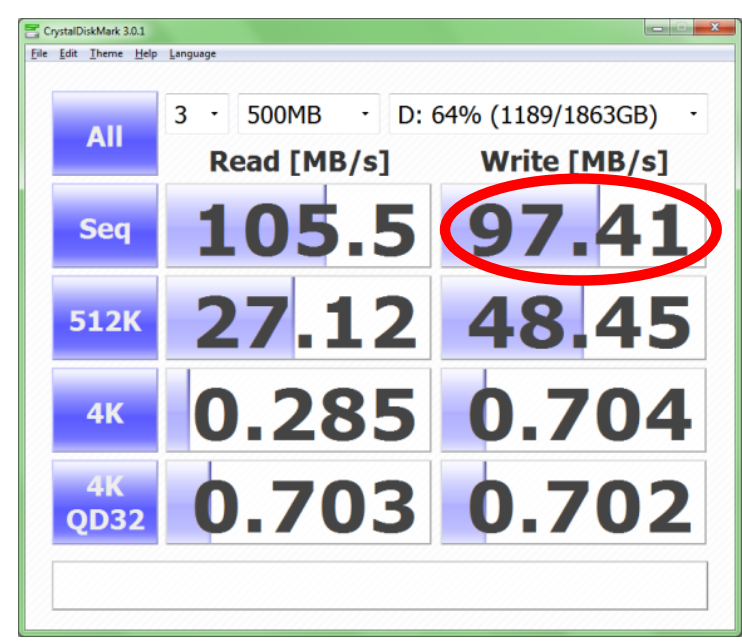

Read and write speed benchmark in $\mathrm{MB} / \mathrm{s}$

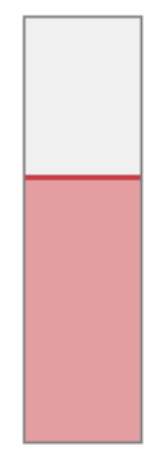

8.3 MB

Average writing speed in $\mathrm{MB} / \mathrm{s}$ during the concurrent recording of 16 television channels on Freeview|HD

Figure 3.6

"Comparing an HDD writing rate to the speed required to record 16 channels in MB/s"

Writing to disk multiple high bitrate recordings also means that space becomes quickly an issue. For instance, with high definition channels having an average bitrate of $10 \mathrm{Mbps}$, this is an average of $1.25 \mathrm{MB}$ every second or $4.4 \mathrm{~GB}$ per hour to be saved for a single HD channel. This trend quickly escalates when multiple channels are recordedas documented in Table 3.3-therefore the duration to keep the videos is directly influenced by the number of channels recorded or by the disk space allowed. With the price per gigabyte decreasing and the average hard disk space around 2 terabytes, it is assumed that, at time of writing, such disk space (2TB or more) is accessible to the public. The prototype system built for this experiment uses a single 2TB hard drive, however, to attain the ideal goal (conceptually nice for the user) of two weeks of recording time multiple hard disks stacked in an array would have been required. 
One can easily imagine that in a near future such configurations will become standard, especially for home theatre machines that do not need large processing power but instead require more disk space due to the increasing quality of the content being received. It would require about $12 \mathrm{~TB}$ of hard disk space to be able to record all Freeview|HD channels for 14 days without interruption. However taking in account the fact that not all channels broadcast $24 \mathrm{~h}$ per day, and because people are not always interested in every channel being offered 6TB of disk space would most likely be sufficient.

The increasing resolution of video content offered, such as high definition channels and the specific broadcast techniques such as interlacing, increase the demand on the CPU (Figure 3.7):

- The video stream is more complex to decode, H.264 requires more processing cycles during playback;

- The de-interlacing filtering that needs to be applied to the decoded frames is more processor intensive due to the higher resolution.

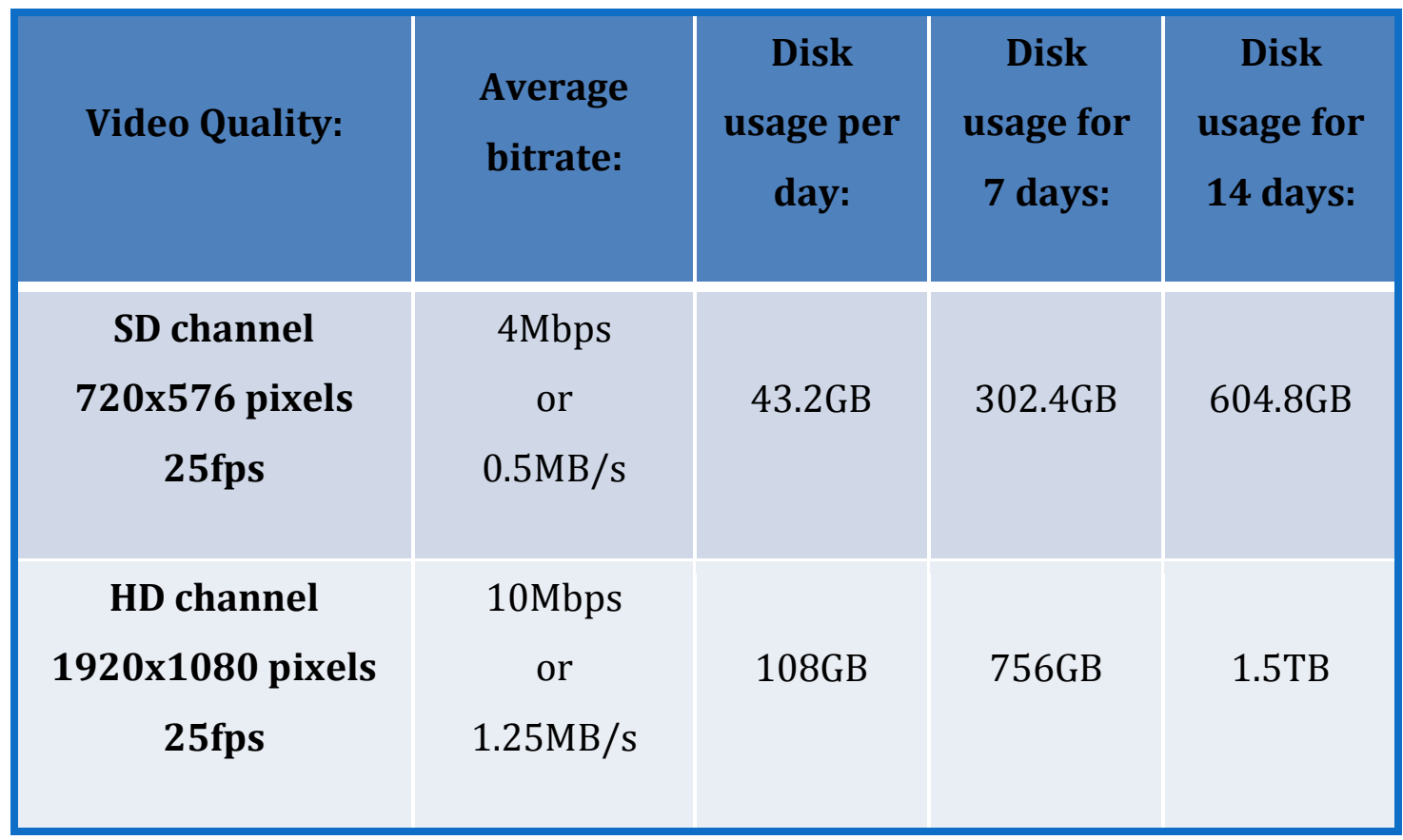

Table 3.3

"Average disk utilisation per TV channel depending on video definition" 
Digital or analogue video can be interlaced or non-interlaced (progressive). Progressive video simply displays whole frames one after the other. Interlacing a video is a technique that doubles the perceived frame rate of analogue TV while consuming half the bandwidth per frame. The idea is to divide a frame into two fields. Each field contains half the horizontal lines of the frame it needs to be assembled back together in order to recompose the final image-as presented in Figure 3.8. This system was created for the early TV sets since they were not capable of displaying complete images at once without showing flickering. To solve this issue, interlaced video takes advantage of the phosphor's afterglow (contained in early cathode ray tube screens) and the persistence of vision effect (Adobe, 2000).

However interlaced video content cannot be displayed on modern progressive scan displays such as computer display monitors or flat TV screens without artifacts known as "combing," especially during scenes with motion. Despite these issues, interlacing is used on all channels offered by Freeview and its competitor SKY, therefore a post processing de-interlacing filter is required in order to display the recordings correctly.

There are many different de-interlacing algorithms available, ranging from a low to high complexity that deliver results that can vary in quality from poor to very good-as shown in Figure 3.9. At the high quality end of the scale there is the "Yadif" algorithm that generates a full picture by taking the odd lines from one field and generating the even lines through a complex algorithm that includes both temporal and special interpolation (VideoLAN, 2011).

VLC (from VideoLAN) is a fren open source multi-platform, multimedia player. Not only does in include the Yadif de-interlace algorithm, it directly embeds many recent codecs such as H.264, HE-AAC and also provides efficient real time video filtering facilities such as the "Yadif" de-interlace filter. For all those reasons, this was the video player framework that was selected for this project. 


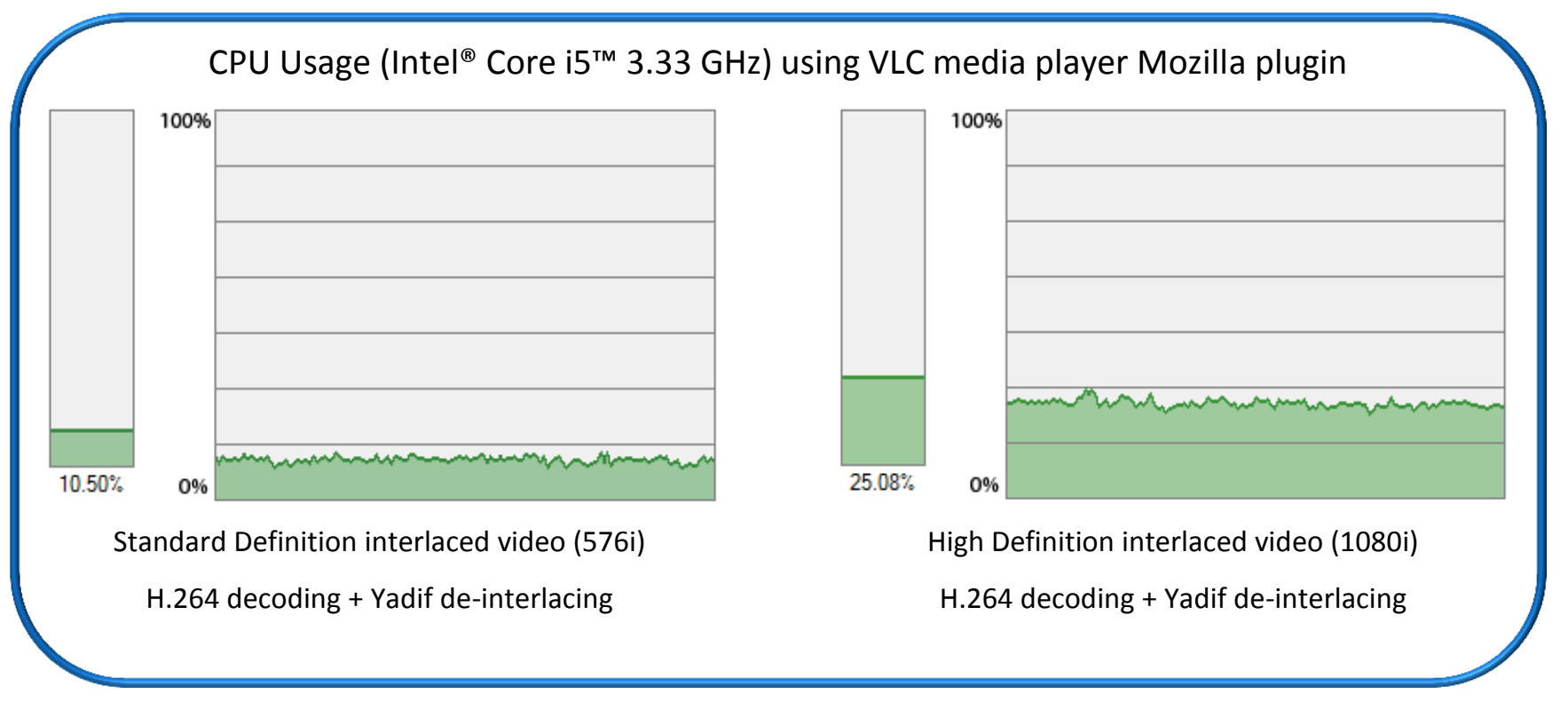

Figure 3.7

"H.264 video decoding and de-interlacing CPU utilisation depending on definition"
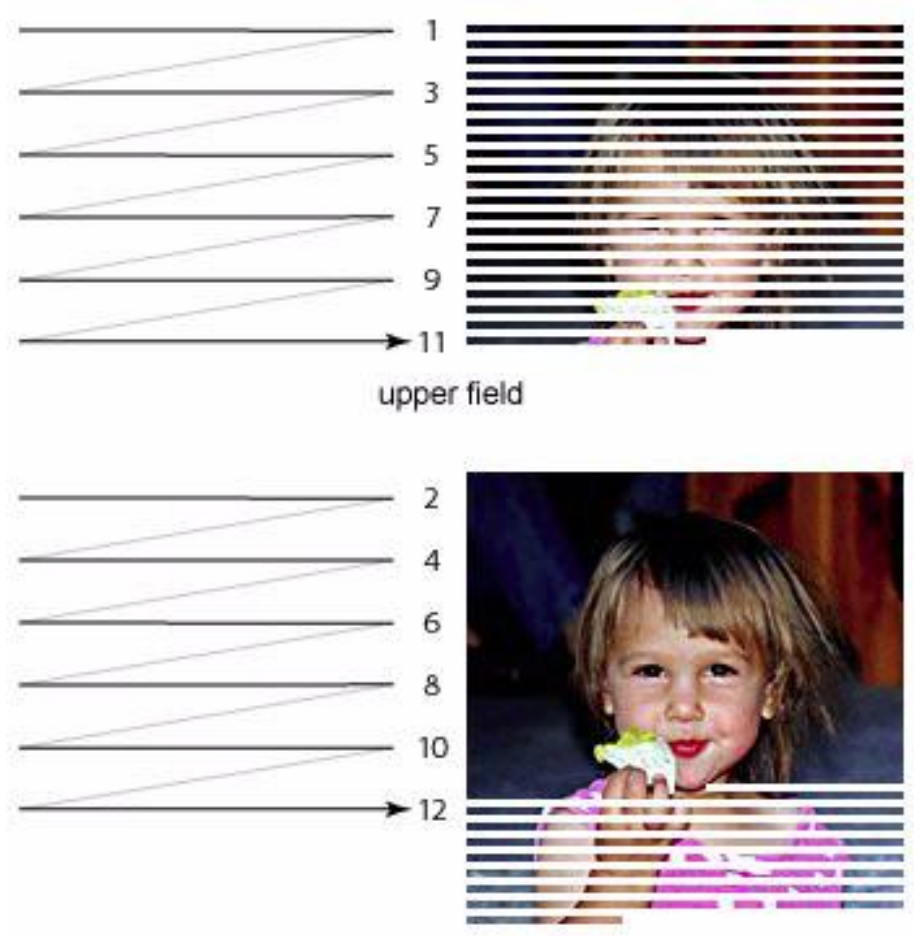

lower field

Figure 3.8

"Interlaced video scanning-Extracted (Adobe, 2000)" 


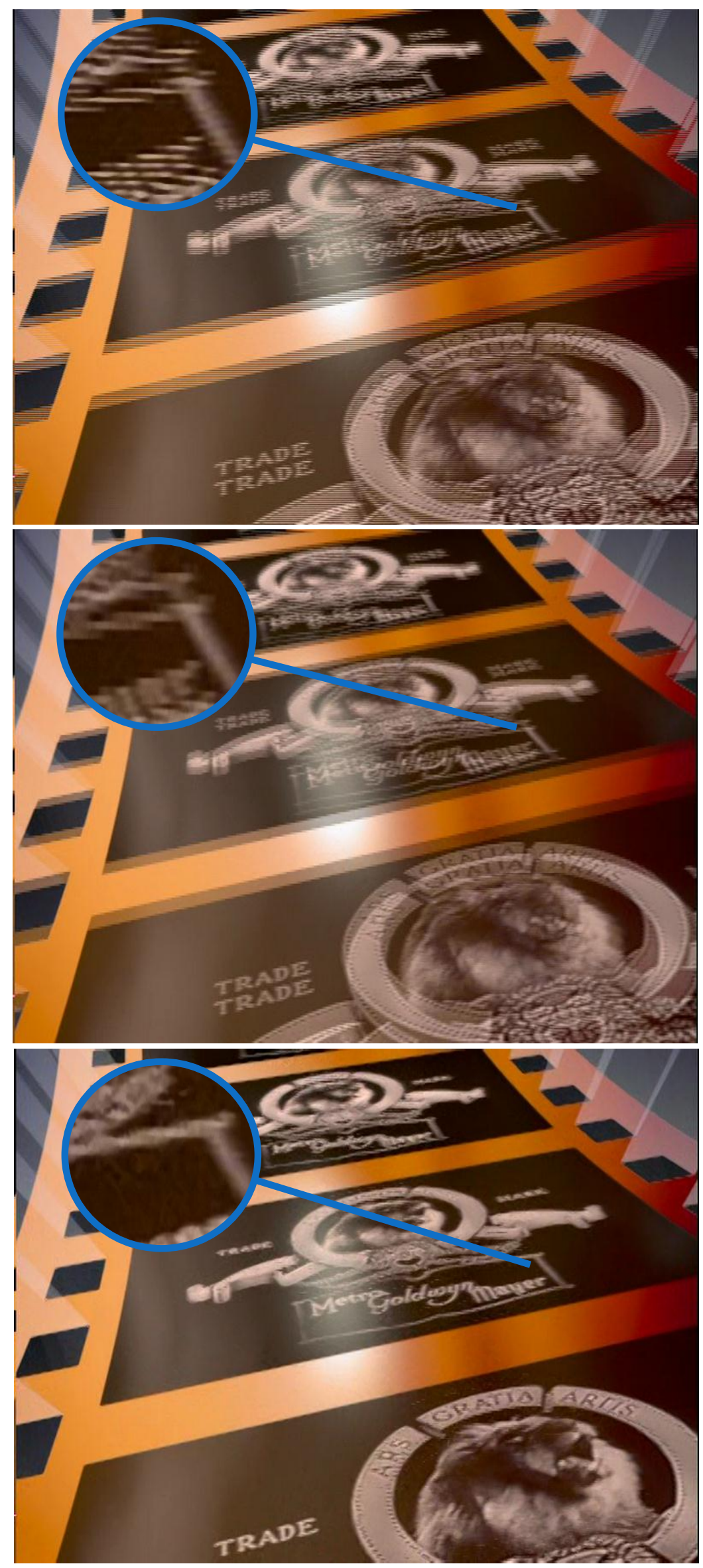

Interlaced content displayed

on a progressive screen.

Blend (full resolution)

de-interlacing example

Yadif de-interlacing example

Figure 3.9

"Comparison between a simple (Blend) and a complex (Yadif) de-interlacing filter-Images extracted (VideoLAN, 2011)" 


\subsubsection{Streaming capabilities}

We now turn our attention to streaming capabilities. Here, being able to consult the video digital library directly (including playback) on the host computer system, which could be plugged into the home television in the living room for instance, is one requirement for RM. Indeed, we seek a solution that goes beyond this by allowing multiple users to simultaneously watch a different show from their own machine (as a client) on the local network from their web browser.

Due to the large file size involved in this project-usually around 4-8GB per recordingtransferring entire files from the recording machine to client's computer before playback is not a practical solution because the file transfer duration would take too long. Therefore, streaming video is the only appropriate option to offer the same user experience as on the main recording machine from a client computer. The fact that Replay Me! uses a digital library software automatically makes the content available to download from the network via any web browser. The RM video player that is based on a VLC plugin for web browsers features the "pseudo streaming" technology of any video hosted by the digital library's web server (Apache).

This "pseudo streaming" technique permits a user to skip to parts of the video not yet received by discarding what has been downloaded so far and initiating a new HTTP request to Apache, asking to start sending the file again, but from a new offset position. The video player then starts playing the content from the offset which is once again progressively downloaded from the server. This technique improves the user experience without requiring the software implementation to shift to an external fully featured video streaming server.

Utilising the VLC media player in this project we can go further than this basic pseudo streaming by replicating some of the behaviour of a dedicated video streaming solution to optimise its bandwidth usage in real time according to the properties of the recording that is streamed. Built in to VLC, the video player has the capability to start to buffer some video frames (which is usually instantaneous) and then, while playing, it can "throttle back" to only download the file at the same rate as required by the video to play, to prevent the network bandwidth being monopolised by a single user. The graph shown in Figure 3.10 compares the streaming of a video recording in high definition against the progressive download of the same file over a gigabit network. It clearly 
demonstrates the bandwidth efficiency exhibited by VLC's advanced implementation of pseudo streaming with any standard web server. This is equivalent to the use of a dedicated streaming server configured with a bandwidth limiting technology such as the one used by Google to host Youtube videos.

The high bitrate of the recordings also directly impacts on the network bandwidth requirements for uninterrupted video streaming. In some cases the speed of the network is the limiting factor in a concurrent streaming environment as shown in Figure 3.11. On ultra-high speed networks, such as $1 \mathrm{Gbps}$, the HDD speed could become once again the limiting factor. Direct video streaming from Replay Me! is possible via the local network, but only the content management aspect of the digital library web interface and the fast video preview are practically accessible from remote locations, due to the limited uploading speed available on residential Internet connections.

In the near future, with the deployment of FTTH (fibre to the home) in New Zealandalso known as the UFB (Ultra Fast Broadband) initiative-remote high definition video streaming without transcoding could technically become possible. However, the scope of remote access of recorded content poses many copyright issues which are beyond this project since we are only interested in investigating what the raw technology can provide to home users. 


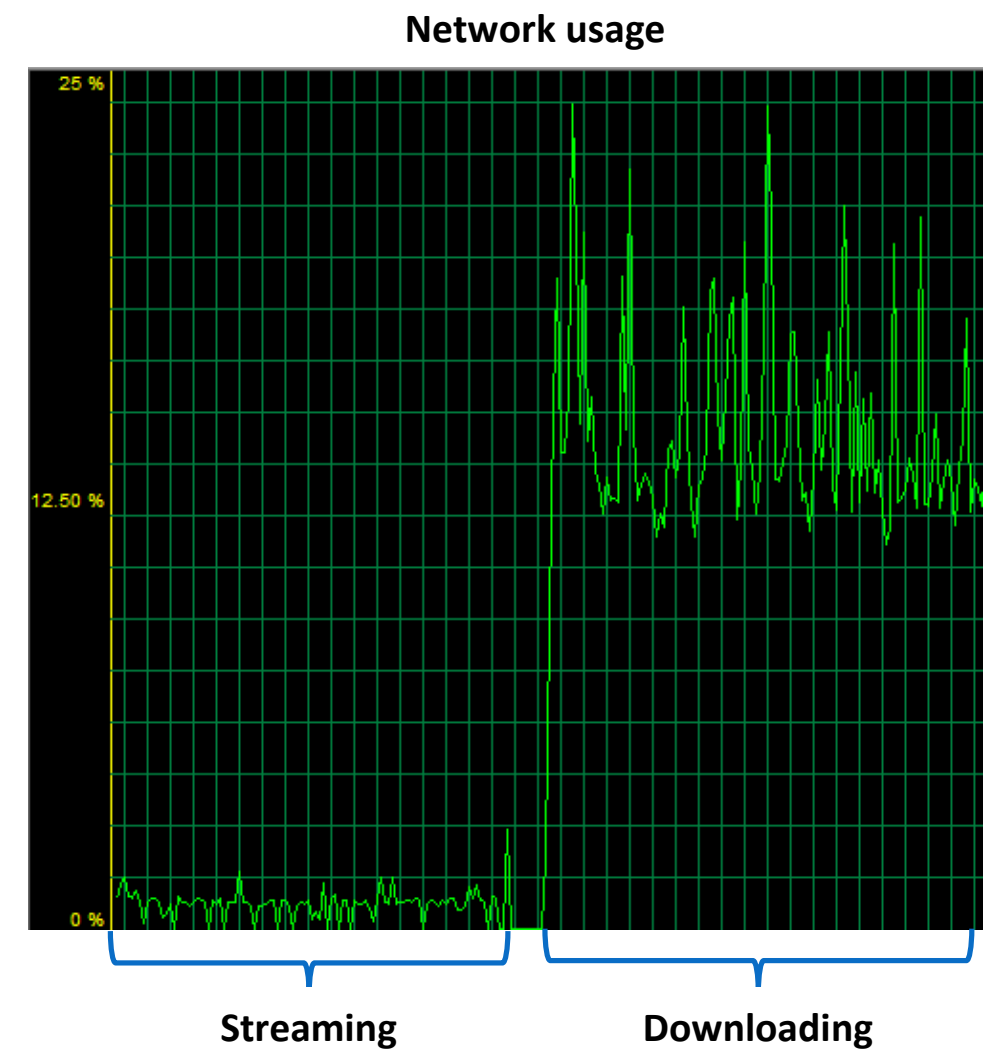

Figure 3.10

"Streaming versus Downloading the same HD video recording on a gigabit network"

\begin{tabular}{|c|c|c|c|c|}
\hline & $\begin{array}{c}\text { Average } \\
\text { stream bitrate }\end{array}$ & $\begin{array}{c}\text { Wifi } 802.11 \mathrm{~g} \\
\text { Max real speed: } \\
22 \mathrm{Mbps}\end{array}$ & $\begin{array}{c}\text { 100BASE-T } \\
\text { Max real speed: } \\
\text { 100Mbps }\end{array}$ & $\begin{array}{c}\text { 1000BASE-T } \\
\text { Max real speed: } \\
\text { 1Gbps }\end{array}$ \\
\hline $\begin{array}{c}\text { High } \\
\text { Definition }\end{array}$ & $\begin{array}{c}4 \mathrm{Mbps} \\
\text { or } \\
0.5 \mathrm{MB} / \mathrm{s}\end{array}$ & $\begin{array}{c}\mathbf{5}^{\mathbf{a}} \max \\
\text { concurrent } \\
\text { streams }\end{array}$ & $\begin{array}{l}25^{\mathbf{a}} \text { max } \\
\text { concurrent } \\
\text { streams }\end{array}$ & $\begin{array}{c}\mathbf{2 5 0} \mathbf{0}^{\mathbf{a}, \mathbf{b}} \max \\
\text { concurrent } \\
\text { streams }\end{array}$ \\
\hline $\begin{array}{l}\text { Standard } \\
\text { Definition }\end{array}$ & $\begin{array}{l}10 \mathrm{Mbps} \\
\text { or } \\
1.25 \mathrm{MB} / \mathrm{s}\end{array}$ & $\begin{array}{c}\mathbf{2}^{\mathbf{a}} \max \\
\text { concurrent } \\
\text { streams }\end{array}$ & $\begin{array}{c}\mathbf{1 0}^{\mathbf{a}} \max \\
\text { concurrent } \\
\text { streams }\end{array}$ & $\begin{array}{c}\mathbf{1 0 0}^{\mathbf{a}, \mathbf{b}} \text { max } \\
\text { concurrent } \\
\text { streams }\end{array}$ \\
\hline
\end{tabular}

Figure 3.11

"Theoretical number of simultaneous video streaming according to the network speed" 


\section{Television recording implementation details}

This project has been implemented in two key parts: the former part has been developed as a plugin for the Media Portal TV Server, and the latter part has been directly integrated into Greenstone digital library software (as explained in more details in Chapter 5). In this chapter more details about how the recording side of Replay Me! works are provided.

\subsection{Media Portal TV Server Plugin}

Media Portal is an open source home media centre constituted of two essential parts. The TV Server handles the TV capturing hardware, EPG, recordings, time-shifting and many other technical aspects of getting access to transmissions. The Media Portal client software is the front-end interface designed for living room TVs that connects to the TV Server. Media Portal is written in C\# and is only available on the Windows platform. As most TV capturing hardware on the market only have drivers and support for Windows, using Media Portal to get access to the TV broadcasts is a good option.

For this project, only the TV Server has been used since it contains everything that is required to record the digital television broadcasted over the air. The TV tuner is a core component of TV Server, and fortunately this software includes the possibility for external developers to write their own plugins in order to enhance its functionality.

In a nutshell, the plugin developed for this project is the bridge between live television streams and the digital library. It communicates with the TV tuner, uses the information from the EPG to segment the live stream into video files and sends the recordings, along with their metadata, to Greenstone. 


\subsection{Listing the possible concurrent recorded channels}

As explained in Chapter 3, a single tuner can record an entire multiplex, but the possible list of channels that can be recorded concurrently depends on the hardware configuration of each machine and the channel selection made by the user. In fact, a computer can be equipped with several TV tuners connected to different antennas pointing to different transmitters. For example, a computer could contain two DVB-T tuners connected to a terrestrial antenna and two other DVB-S tuners connected to a satellite dish. Having such flexibility is useful as TV broadcasters expand the number of available channels, however it can also lead to complicated configurations when determining the list of possible channels that can be recorded. This is the most likely reason the Microsoft Windows Media Center has chosen to oversimplify the choices available to the user, by restricting recording to one channel per tuner.

Replay Me! goes beyond what is on offer in the Windows Media Centre yet. It hides the inherent complexity to the end user by simply displaying two lists of channels on the main tab of its configuration panel-as picture on Figure 4.1:

- The list of available channels from all the detected tuners (on the left);

- The list of channels selected by the user to be recorded (on the right).

Picking channels to record is achieved by the user selecting channels in the left-hand list and moving them (using the arrow buttons) to the right.

As each selection is made, Replay Me! re-evaluates what channels can still be offered to the user:

- When multiple channels are selected to be recorded, the system tries to timeshift (record to temporary files) all the selected channels to guarantee that the signal strength is adequate for this selection.

- At the same time, RM tests all the non-selected channels remaining in the list to find any channels that cannot be recorded due to both the user's selection and the tuners' configurations. All the channels that cannot be added to the recording list due to hardware limitations are dynamically removed from the available channels list. This is to prevent any selection that is technically impossible to 
record simultaneously with the current hardware and the number of multiplexes/transponders broadcasted in the area of reception.

Once the selection is completed, the user can launch the concurrent recording, segmenting and indexing of the selected TV channels by clicking on the "Start recording" button.

Since the entire operation runs as a Windows service, it is possible to quit this window and leave the entire process running in the background.

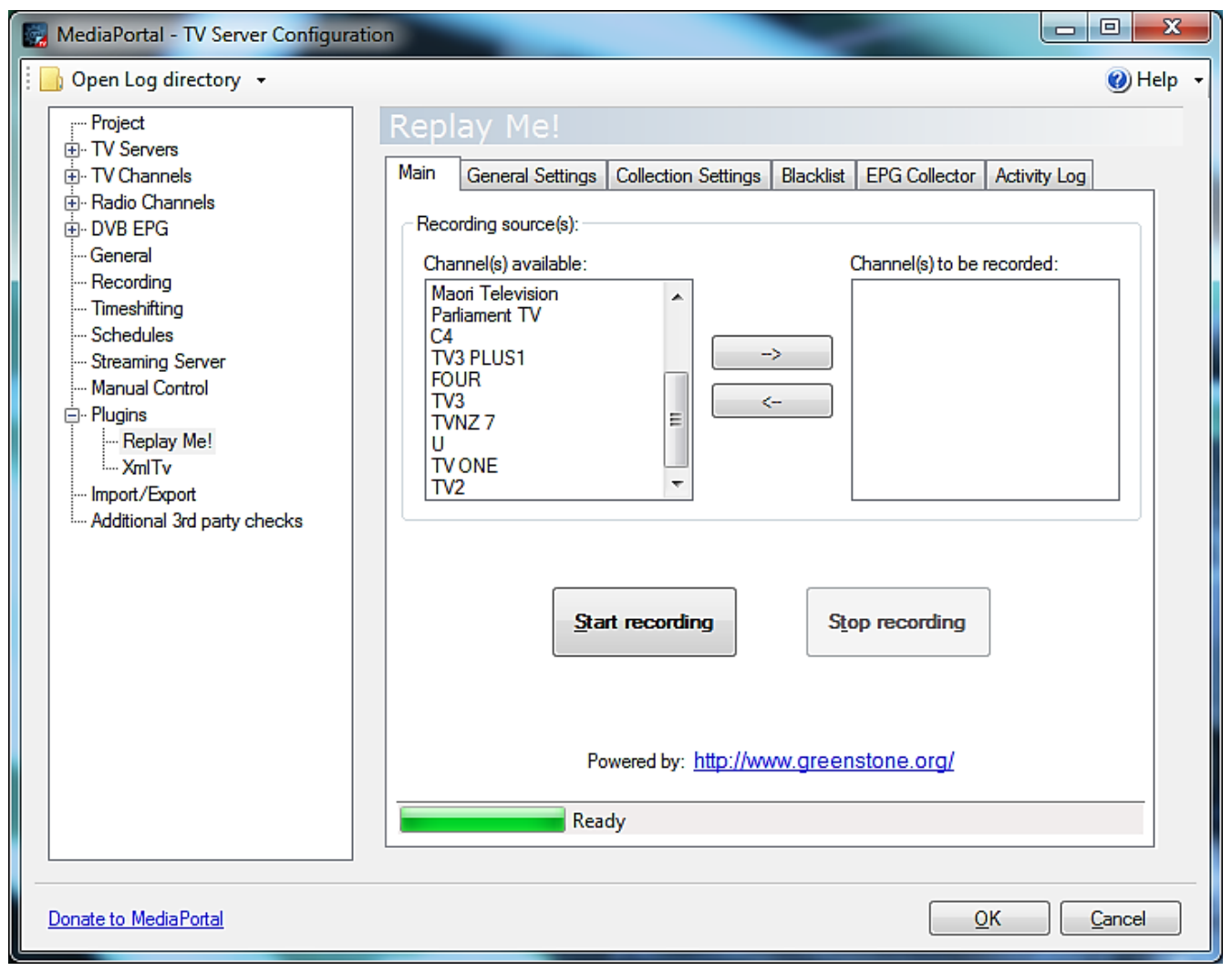

Figure 4.1

"Replay Me! MediaPortal TV Server Plugin-Main tab" 


\subsection{Getting access to the EPG off-the-air}

The electronic programme guide data is a critical piece of information for this project. Often this information can be found on the Internet from various non-official sources but Replay Me! aims to be as standalone as possible, therefore the EPG is directly extracted from the antenna feed.

In New Zealand, Freeview|HD does not use the DVB EIT (Event Information Table) standard to broadcast the entire EPG but has selected MHEG-5-a standard specialised in TV middleware and standardised by the Multimedia and Hypermedia Experts group-instead, to broadcast their entire EPG as shown earlier in Table 3.2. The New Zealand MHEG-5 profile is similar to the one used in United Kingdom with only some minor modifications. This solution has been selected by Freeview in order to have a consistent interface for the end user during the consultation of the EPG from one set up box brand to another.

Due to this non-uniformity between countries, the MediaPortal TV Server cannot import the full NZ EPG from the antenna seamlessly. To solve this issue, Replay Me! uses another external open source software called "EPG Collector" which is capable of getting access to the EPG in various formats from many countries where broadcasters do not use the DVB standard to broadcast their EPG. It connects directly to a TV tuner (Terrestrial, Satellite, Cable) and tunes to a specified frequency-depending on the country and region of residence-to collect the EPG which is then written to an XMLTV file (an open XML based EPG format).

The downside of using this method is that it requires one of the hardware TV tuners to be continually committed to extract the EPG from the live feed. The trade-off for this project was considered worthwhile as this enables us to get the data from the most official source without any Internet connection requirement: the broadcasted TV stream itself as it happens. Since Freeview|HD only uses 3 multiplexes as detailed in Chapter 3, and our prototype machine is equipped with two internal dual-tuners, this leaves one TV tuner free to be dedicated to this purpose.

In the TV Server configuration, the Replay Me! plugin integrates a tab dedicated to this activity called the "EPG Collector", letting the user configure the software according to their particular hardware setup. A tick box is also present to periodically launch EPG 
Collector in the background in order to collect an updated set of 7days EPG data, which is then automatically imported into MediaPortal's internal EPG database-as pictured in Figure 4.2.

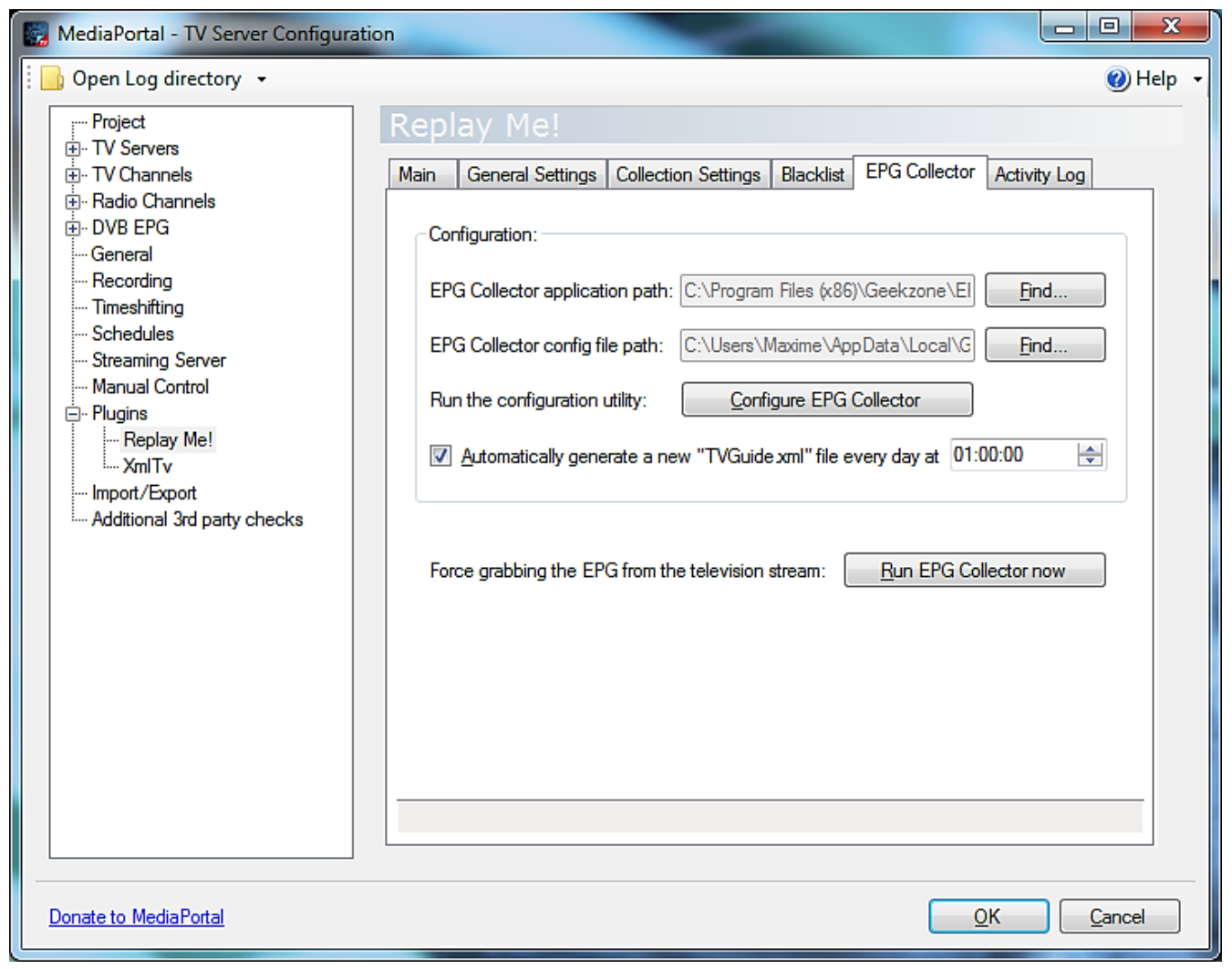

Figure 4.2

“Replay Me! MediaPortal TV Server Plugin-EPG Collector tab" 


\subsection{Automatic video segmentation}

The recording schedules are the foundation to the algorithm in RM that takes care of the segmentation of the live television stream into video files. The idea is to automatically create recording schedules for the following 48 hours for every show on the specified channels from Figure 4.1, using the timing information gathered from the EPG.

The software TV tuner (inside Media Portal TV Server) then checks the list of recording schedules periodically and automatically starts to write the recorded shows to disk.

New schedules can be created in two situations:

- From the moment the "Start recording" button is clicked;

- Every day as long as recording is not manually halted.

At a more technical level, Replay Me! records the time when someone clicks on the button that initiates the recordings. This information is used to generate more recording schedules automatically every 24 hours until the user clicks on the "Stop recording" button. When triggered, the algorithm will first check if the system is in recording mode-by having the "Start recording" button pressed. It will then create two arrays: one for listing the available channels on the system and another to list the possible television programs on each channel from the internal EPG database.

These arrays are used to find two pieces of information:

- The current program being broadcasted on the channels that should be recorded. Once the current programs are found, they are added to the recording schedules' database.

- All the programmes' schedules for the following 48 hours for each of the channels selected for recording. Once found, these lists of recording schedules are also added to the recording database.

From that moment on, the system has the current show plus two days of recording schedules ahead for each of the channels selected in memory. The last step is to initiate the recording hardware in order to start the recordings straightaway-the stream segmentation of multiple TV channels is now initiated. 
This scheme will run continuously until someone manually stops the process by clicking the "Stop recording" button in the plugin control panel. Doing so will stop the current recordings and delete all further recording schedules. It will also prevent new recording schedules from being automatically created. 


\subsection{Exporting the finished recordings with metadata}

With the introduction of this plugin, the Media Portal TV Server is now controlled by the Replay Me! in order to segment the live broadcast into video files. Once finished, these video recordings are ready to be sent to the digital library but metadata from the program guide still needs to be converted into a format usable by Greenstone-the digital library software used for this project (Witten, et al., 2010). For this purpose, the plugin implements a method designed to write the associated metadata (title, description, channel, broadcast date and time, etc.) into an XML file (called metadata.xml) formatted for Greenstone in the same directory where the video files are written to disk. An example of this file is shown in Figure 4.3.

A Greenstone metadata XML file can contain the associated metadata for one or more files within the directory it resides. For that reason, the algorithm first tries to load an existing metadata.xml file if one was already created from a previous recording. If no metadata file is present, it creates a new one by writing the required preamble such as the XML version, the encoding, the document type and the root element.

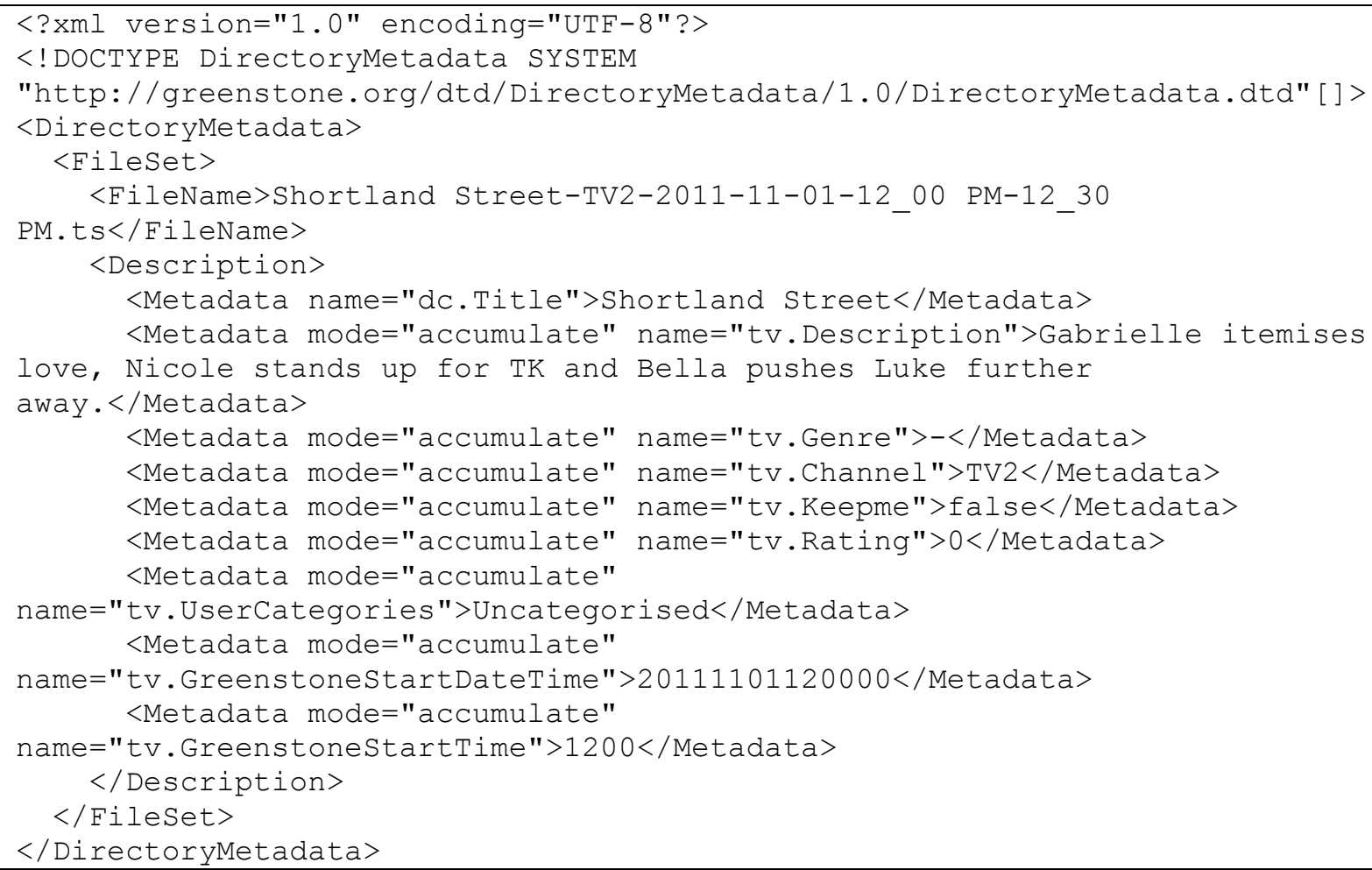

Figure 4.3

"Greenstone metadata.xml file example" 
Then the plugin appends the new child elements that describe the metadata information from the internal program guide data for the current recording. For this purpose, a new metadata prefix has been created for the metadata fields to separate the EPG's metadata from the metadata that can be manually added through other parts of Greenstone such as the Librarian Interface (Witten I. H., 2004). The metadata automatically extracted from the program guide uses the "tv" prefix in contrast to the more usual "dc" prefix associated with the Dublin Core metadata set.

This method will be executed for every recording so that they can be imported and indexed into Greenstone with their associated metadata.

At this point, the television shows being saved to disk with their metadata are written to a temporary folder that is not seen by the digital library yet. In order for Greenstone to see this content it needs to be moved into the video digital library's special import folder.

For this to work, every time a new recoding is finished the Media Portal plugin will start by preparing the import folder by moving the recorded video(s) along with the metadata XML file to it. Greenstone can then be launched in the background using a separate thread so as to not interfere with the continuous recording operation. The newly recorded videos are processed and ingested from this import folder, as explained in more details in Chapter 5.

Putting all this together, the Replay Me! video collection is now automatically populated by new recordings as soon as they arrive in the digital library-which is around 5 minutes after the shows being aired are finished. 


\subsection{Recordings queuing system}

Each time that a new recording is finished, it is sent to the digital library for processing (information and thumbnail extraction) during the importing stage, however Greenstone may still be processing one or more previous videos. The system should prevent the concurrent execution of Greenstone's import procedure to avoid importing files that may already be in the process of being imported. This scenario can happen for instance when a long recording is followed by a short one, or simply when two shows finishes at the same time on different channels.

A queuing system was implemented to cope with these situations, designed to wait for an active Greenstone process to finish before preparing the import folder again for a new import stage. In fact, while the digital library is still in the process of importing previous recordings, (as mentioned previously) new ones are recorded inside a temporary folder and only moved to the importing folder when Greenstone is not being used anymore. In this situation, every time a new recording is finished, its complete file path is added to an array.

When the digital library finishes the processing of any previous video(s), the algorithm will first move all the waiting recordings with their metadata into the import folder and launch Greenstone's building stage again to process the new batch of TV shows. From this moment all new shows finished are again placed in the queue. This procedure is described in Figure 4.4.

This method will, in principle, only run when multiple shows finishes at the same time since our system is optimised enough to process the recordings in the queue faster than real-time. 


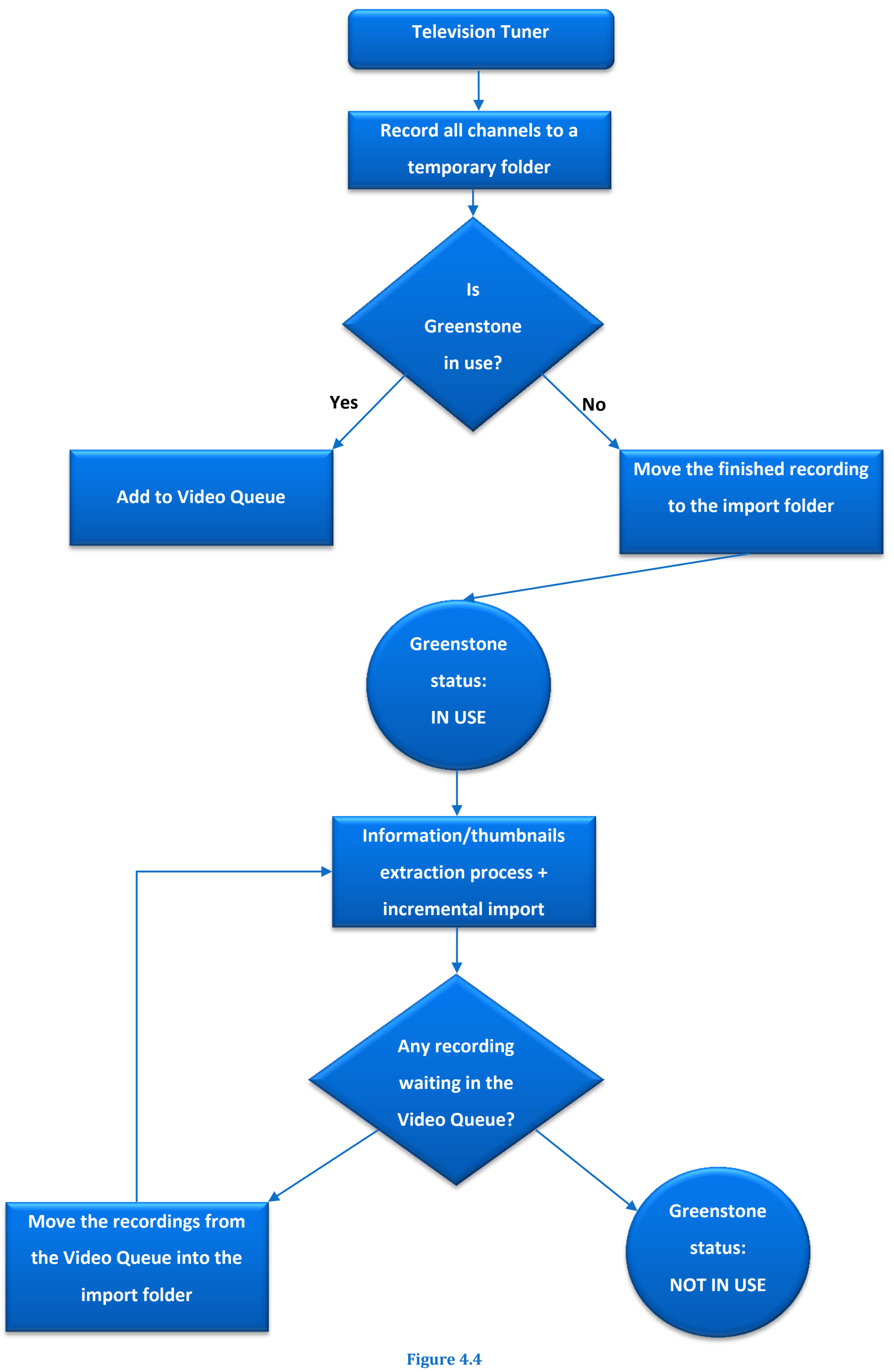

“Replay Me!'s recordings queuing system diagram" 


\subsection{Recording schedules management and disk space optimisation}

Having TV shows that are not aired exactly at the times that they were scheduled is a common situation. Similar to systems like MySKY HDi, to prevent the situation where a recorded show misses the start or the end it was actually broadcast, Replay Me! adds in a pre-recording and post-recording time/overlap for every show as defined in the Media Portal plugin and shown in Figure 4.5.

Broadcasters use very high bitrates for the transmissions of television channels as detailed in Figure 3.5 and Table 3.3. Since this project deals with large amounts of data, the disk usage is managed in several ways:

- Replay Me! does not actually copy any video recording from one directory to another-it always moves or hard-links (as appropriate), in order to optimise the speed of the processing but most importantly to avoid file fragmentation as much as possible. Moreover, temporary recording directories, the digital library's import folder and the final destination's directories are always located on the same physical hard disk. ${ }^{4}$

- Within Greenstone, there are many settings available to import files. Replay Me! uses Lucene-an indexing engine from the Apache foundation-which enables incremental building and deleting of the television recordings without having to re-create the video digital library from scratch every time a new recording is added.

- The recordings are only stored for a defined period-the "time window" as explained in the introduction (Section 1.1)-before getting automatically deleted from the video digital library. The actual deleting date is calculated from the broadcasted date stored in the metadata of each recording. Users can define this retention period by selecting a number of days or weeks as seen in Figure 4.5; this change is automatically taken into account.

To effectively delete an old recording from the video collection, the plugin writes a special XML file that controls the importing process of Greenstone. This file is called manifest.xml and, it contains the document IDs of the videos that should be deleted (or added, or re-indexed, but only deletion is needed in this case) from the digital library. Every time a recording finishes, Greenstone is launched with

\footnotetext{
${ }^{4}$ The use of multiple HDD can only be optimised for this project if configured as a RAID array.
} 
this setting file to delete any expired recordings prior to importing new TV shows to the Replay Me! collection.

- Recall from Chapter 3 that RM does not transcode (recompress) the videos, the only way, therefore, to optimise file space is to prevent the recording of unwanted TV shows in the first place. For this reason a blacklist of TV shows that can be configured from the plugin-as seen in Figure 4.6-was implemented. Technically, during the recording schedules creation process (as explained in the Section 4.4), the system will compare the name of each show before being added it into either the recording schedules or not.

This is useful to avoid disk space consuming long commercial programs-also called "Infomercials." But more importantly, it allows for the efficient recording of channels that do not broadcast programmes 24 hours a day. For example Maori Television only broadcasts programmes from around $2 \mathrm{pm}$ to $11 \mathrm{pm}$. The rest of the time, all that is that displayed is the Maori TV logo with background music punctuated with intermittent commercials that appear in the EPG as a long show called "Maori television." Similar patterns are also found on other channels. This simple functionality is an efficient way of allowing RM to record more channels, or increase the retention time of existing recording. 


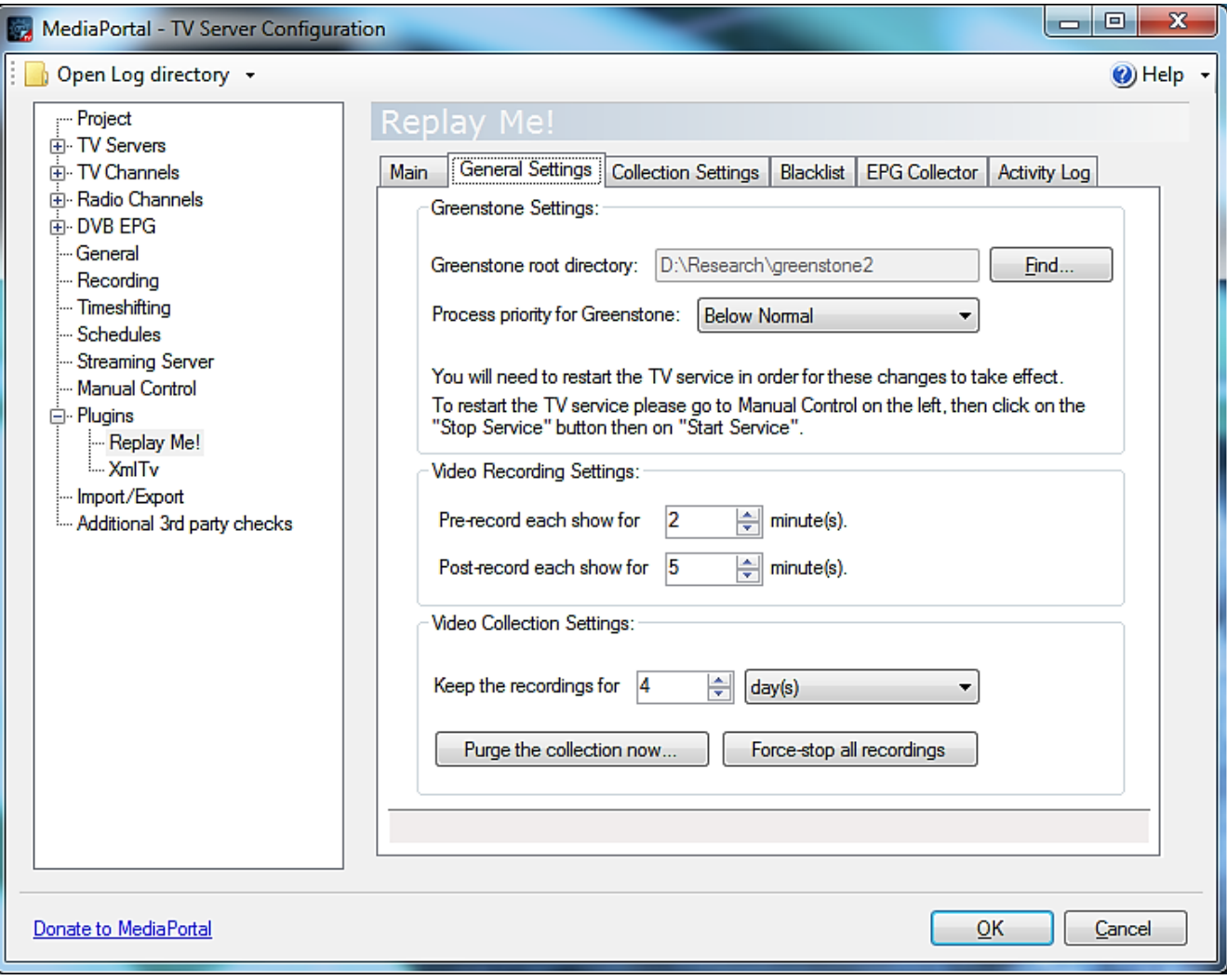

Figure 4.5

"Replay Me! MediaPortal TV Server Plugin—General Settings tab"

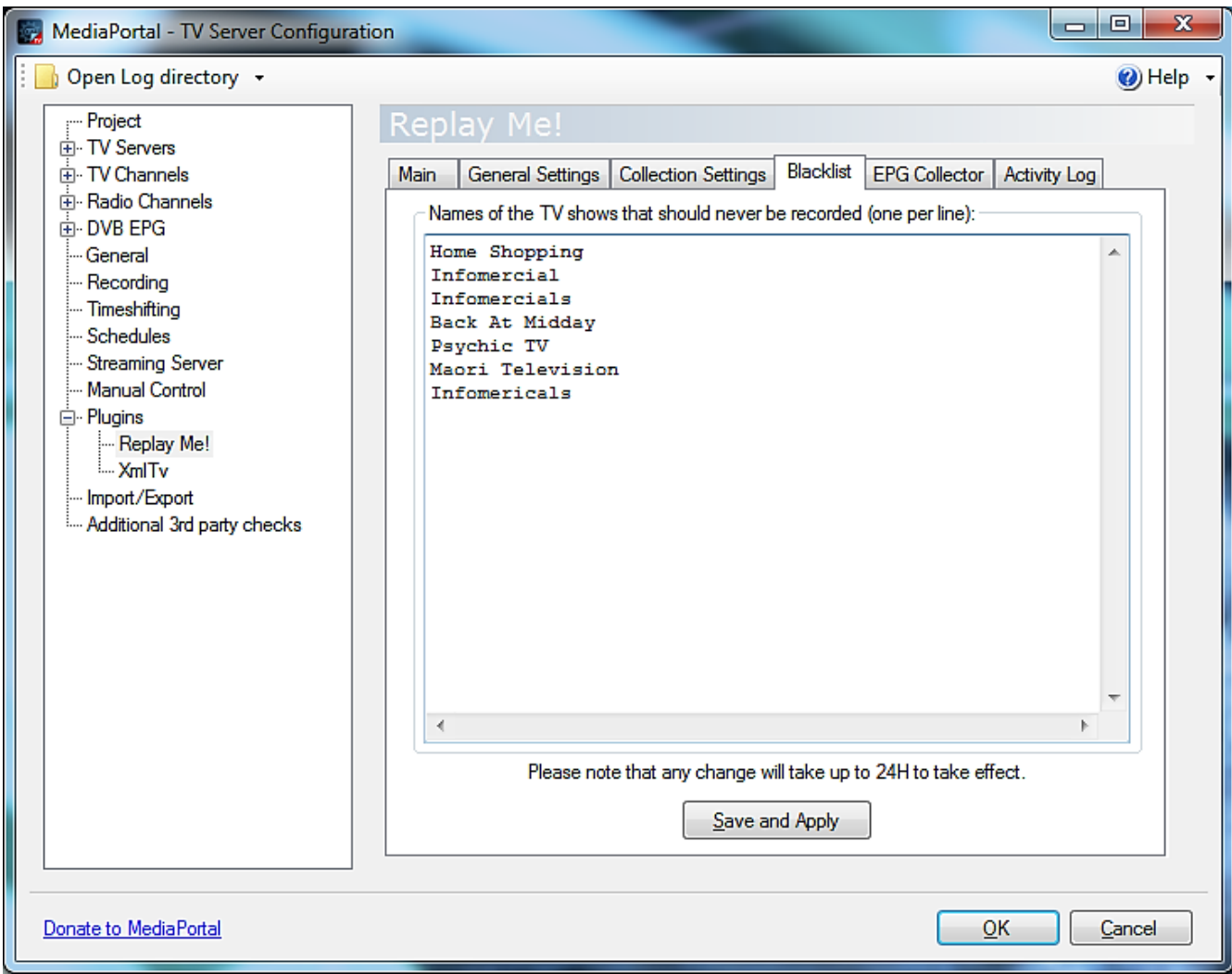

Figure 4.6

“Replay Me! MediaPortal TV Server Plugin-Blacklist tab” 


\subsection{Advanced configuration and activity reporting}

Precise control of Greenstone's importing method can be adjusted directly from the "Collection Settings" tab-as pictured in Figure 4.7. This allows advanced configuration of the streaming technique. In our case a "direct streaming" option which uses the original recording video file for pseudo streaming was created. It is also possible to personalise some parameters that influence the way all recordings appear during searching or browsing the digital library, however the main act of customisation is achieved directly within Greenstone which is detailed in the following chapter.

Since the digital library runs in the background, Replay Me! logs all the output from Greenstone which is accessible from the "Activity Log" tab in the plugin-pictured in Figure 4.8. This enables power users to see the details of how each show was processed and incrementally imported, which is useful for debugging purposes. This log file can be automatically deleted everyday if the option is selected in order to prevent it growing too large.

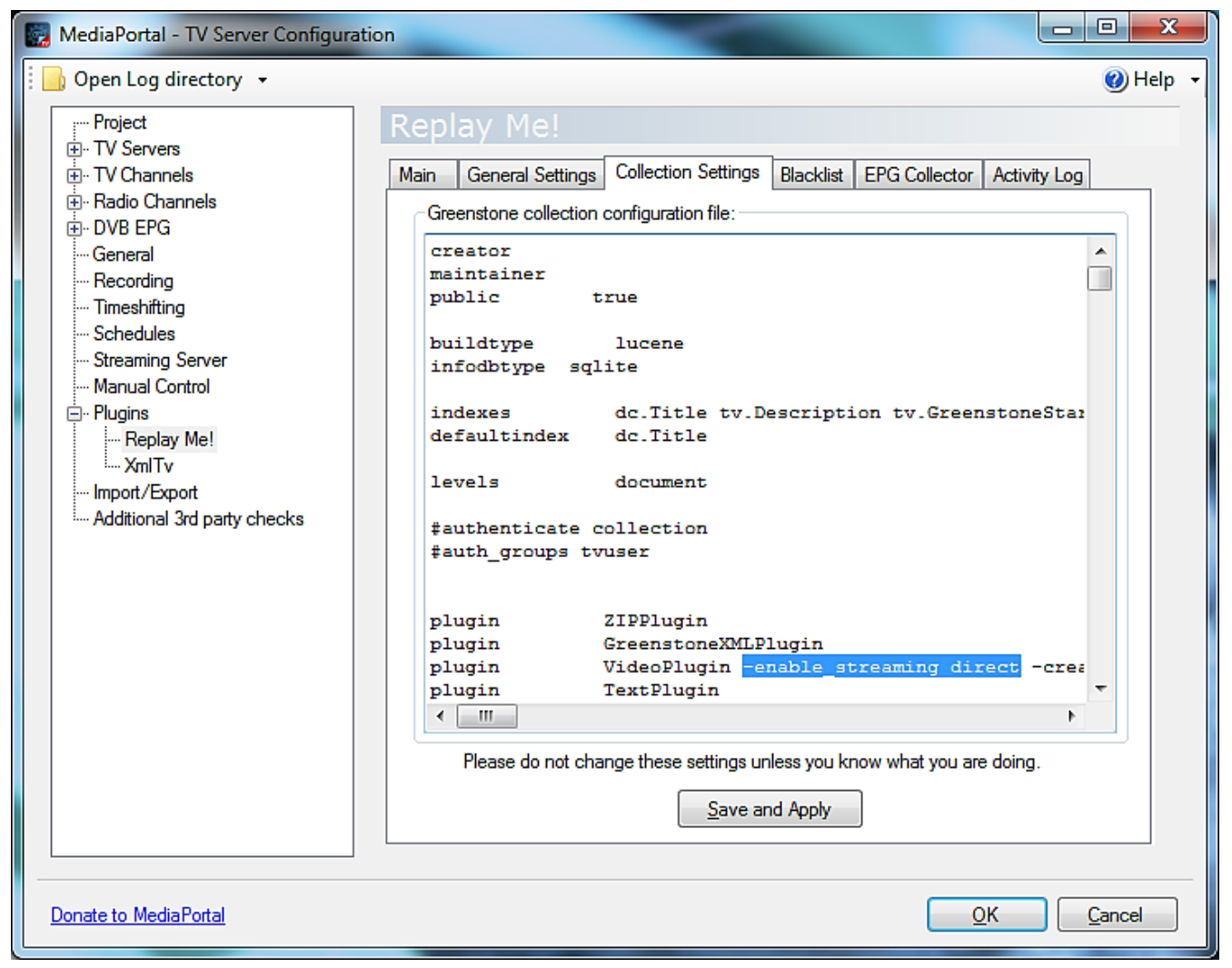

Figure 4.7

“Replay Me! MediaPortal TV Server Plugin—Collection Settings tab” 


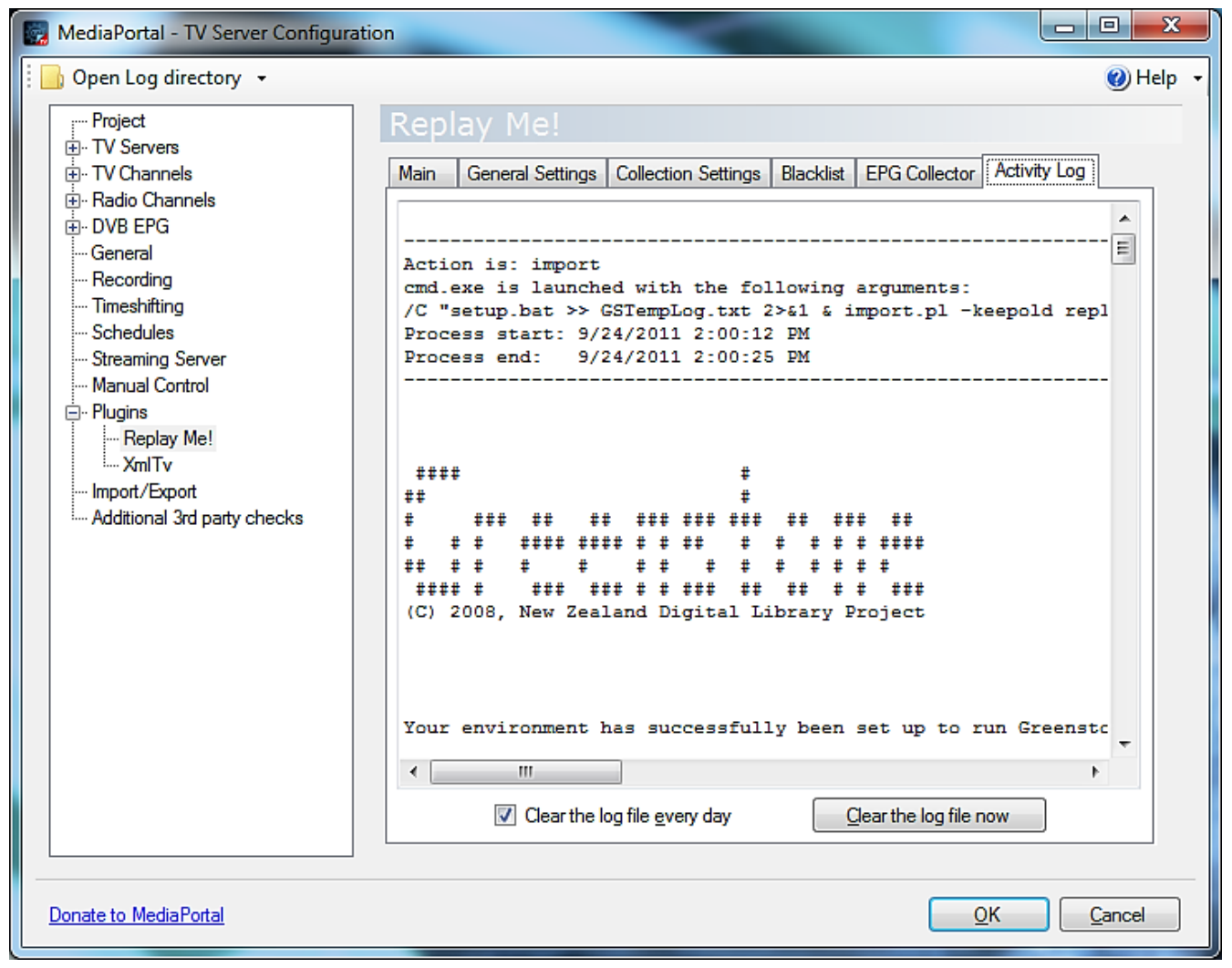

Figure 4.8

"Replay Me! MediaPortal TV Server Plugin-Activity Log tab" 


\section{Digital Library design}

Another significant part of this project has been implemented directly into Greenstone digital library software itself. This chapter first introduces some key concepts of the Greenstone toolkit, and then the modifications made to this digital library software to allow the processing and displaying of television recordings according to the Replay Me! requirements are explained.

\subsection{Greenstone Digital Library}

Greenstone is a complete software toolkit that was initiated by the New Zealand Digital Library Project at the University of Waikato. The aim of the software is to create a versatile system that can be used to organise a set of documents in any format into a digital library with full text searching. Translated into more than 45 languages it is widely used internationally. UNESCO (in a joint project with Waikato) is also promoting its use in developing countries (New Zealand Digital Library Project, 2011). Greenstone runs on multiple platforms, is freely available, and-germane to our needs-designed to be an extensible solution.

A key concept in Greenstone is a collection which can contain virtually any number of documents that are organised in a specific manner that is specifically designed to meet the needs of its users. Documents, from which collections are made of, can be of any type (text, audio, video, etc.); they may have alternative representations and associated files. Metadata is the descriptive information that is associated with each document. In order to be presented to the users, each collection must undergo a building process that once initiated, is completely automated. During the first stages of the building phase, each new document is processed by one of Greenstone's plugins that specialises in that type of document (Word document, HTML file, Video file, etc.) adding it to the collection. They will be referred to as document processing plugins, to differentiate them from the plugin architecture used in Media Portal. Then, the latter part of the building process creates a self-sufficient set of files consisting of the search indexes and the browsing structure necessary to access the collection. From this time on, only a rebuilding process is executed to add new (and also expired) documents to a collection (Witten, et al., 2010). 


\subsection{Processing new television recordings}

To sum up the digital library side of Replay Me!, everything has been implemented in a single Greenstone collection, and the documents are in fact the television recordings. The associated files are the thumbnails of multiple sizes (to display alternate representations at run time) generated during the importing process. The associated metadata is the descriptive information gathered from the EPG and the data automatically extracted from each video recording, such as whether it is standard or high definition.

There is already a multitude of document processing plugins available which allows Greenstone to deal with many different types of file formats. One of them is a powerful video plugin specifically designed to process files extracted from DVDs. However, it did not include provision for the processing of video streams recorded from digital television broadcasts. Therefore the video plugin was modified to match the requirements of Replay Me!. Fortunately, this is not only an improvement for RM, since most of the new functionality is also useful for other types of video formats.

In our previous proof of concept project, a transcoding solution was implemented into this plugin in order to recompress each video recording as it arrived in Greenstone, in order to reduce the space require to store them. Having established that this technique was not scalable to record many TV channels concurrently, a "direct streaming" solution was instead adopted. As broadcast recordings come in a form that is already suitable for streaming, they are stored and then used in the user interface without any modifications. The benefit of working from the original streams is that all the additional data that is contained in each original TV recording is kept-such as the full image resolution, the subtitles, and any multiple audio tracks. This extra material is then analysed by the upgraded document processing plugin in order to extract more metadata in addition to the information from the electronic programme guide.

In order to deal with various possible stream formats, an open source software application specialised in extracting multimedia description called MediaInfo is used. Some of the extracted information contributes to provide better video file descriptions for the digital video library, such as the codec used for the video and audio tracks, the frame rate, and the precise video file size among many other technical metadata fields. New information can also be calculated (derived) from this data, for example the quality 
of the video (HD or SD) can be deduced from the video height and width, similarly the presence of subtitles or multiple audio tracks can be detected, and the approximate transfer time of a TV show from the video library to another computer can be calculated.

Textual surrogates are not always very effective at giving a good sense of multimedia content, however some of its graphical elements can provide cues to users that help them to decide which documents to investigate further (Witten, et al., 2010). To provide alternate representations of the videos, thumbnails of different sizes are extracted from the recorded TV broadcasts, however, the fact that we work from files recorded off-theair poses additional issues:

- The codec used for compression (H.264) is not designed for easy image extraction from any offset, as explained in Section 3.1;

- The transport medium (over-the-air broadcasting) does not always provide error-free recordings, as detailed in Section 3.2.

The original video processing plugin was designed to grab one of the very first frames from the video as a thumbnail. This is practical for videos files coming from DVDs, which are free of errors and are not compressed in MPEG-4 AVC. Compare this with broadcasted TV recordings where, due to the variability of the exact start time of the show, taking an image from the beginning of the stream will not be descriptive of the actual content since it will be an image taken from the previously aired show.

Therefore it was decided to extract an image at approximately $50 \%$ of the length of the recording. Due to the large size of the videos the next complication it that implemented naively this process takes too long. This is due to the fact that H.264 compression heavily depends on inter-frames information, and the command line open source software utility used by Greenstone to extract the images was actually reading half of the video from the start, decoding all the frames in order to get a valid image at the precise point of extraction requested. The operation took close to real-time to accomplish, requiring a high amount of resources from the CPU to decode thousands of frames that are ultimately discarded. In the case of a movie, for example, the extraction of a single frame could take more than an hour.

To solve this issue, the extraction process was re-engineered to first seek into half of the video by using the appropriate byte offset, and then from this point, only decode a frame. 
This technique is nearly instantaneous. However the resulting image was always either black or grey because the decoder did not have enough information to fully "reconstruct" a frame. To resolve this final issue and to make sure that the thumbnail extraction can also recover from transport errors in the stream, the byte-seek into the video was adjusted to first to pick a point slightly before our ideal frame, and then read the video normally for a few seconds (i.e. decode each frame sequentially) before extracting a complete valid image that is then scaled down to an appropriate size.

Replay Me! also generates another multimedia surrogate by extracting frames at regular interval from each recording in order to generate a "fast preview" on demand option for the user during searching or browsing. For this function, an open source package called "The Movie Thumbnailer" was tweaked and optimised in order to match our requirements. The idea is to do the same as with normal thumbnail extraction, by seeking into the file using byte offsets and then decoding some frames to make sure that blank frames are always avoided. But this software goes further by doing some image analysis such as edge detection and blur evasion techniques in order to extract frames that contain a certain amount of detail since they are more likely to be interesting-as pictured in Figure 5.1. Processing time is dependent on the length of the video recording, but overall this process that takes most of the time compared to the other phases explained earlier. Even so, this is still reasonable since it takes less than 5 minutes to process a 2 hours show in HD. 


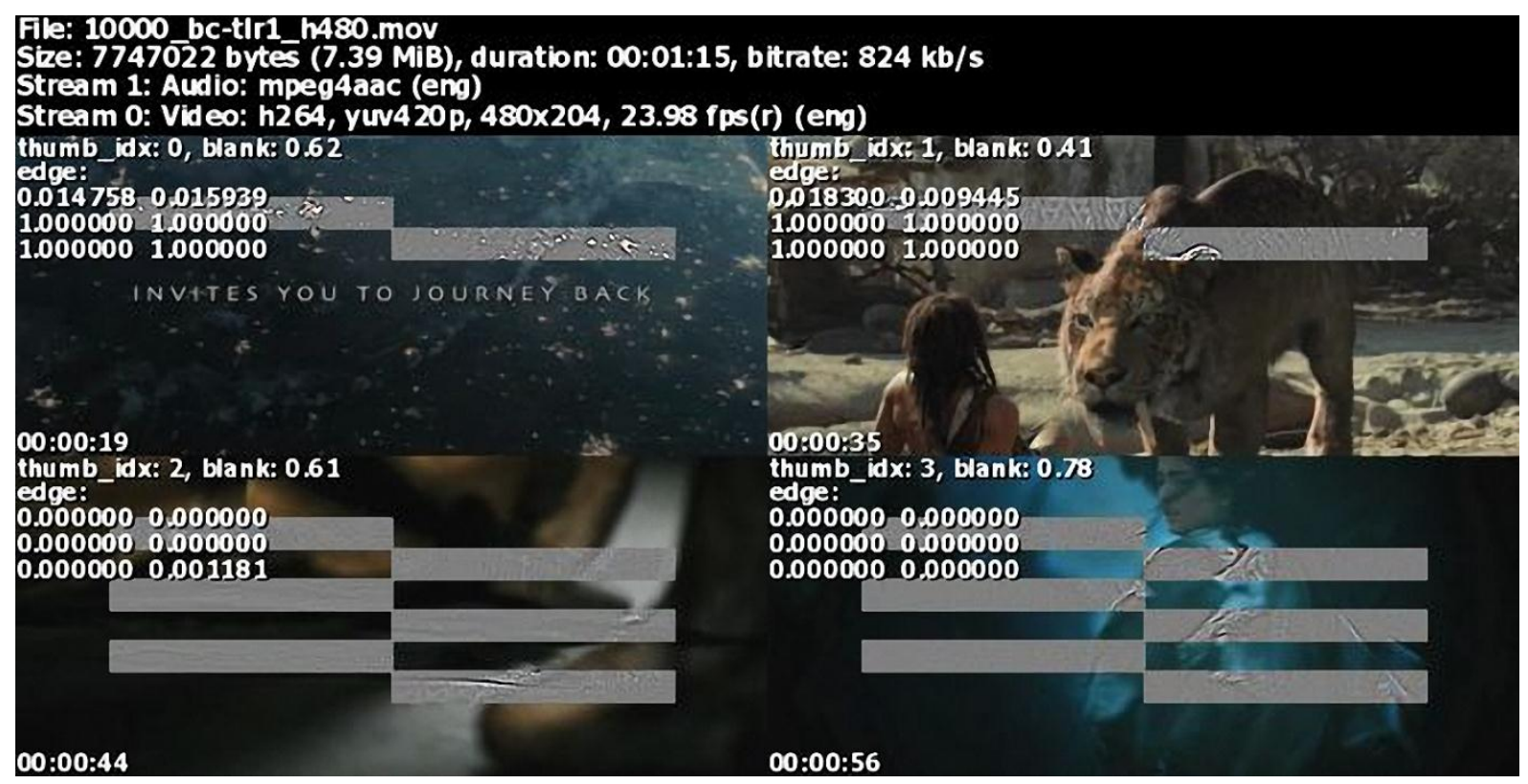

Figure 5.1

"The Movie Thumbnailer: edge detection inner workings example"-Reproduced (Tuitfun, 2011) 


\subsection{Creating the searching index and the browsing structure}

With the processing stage completed, the search index and the browsing structures must be created in order to make the digital video collection work. Greenstone offers many options regarding the type of search index to use, such as MG (Witten, et al., 1999) and Lucene (McCandless, et al., 2010). The latter indexer was chosen because it supports incremental building and deleting of new documents which is more efficient for the large video files that we are dealing with in this project.

Greenstone also offers a choice of database systems in order to store metadata. Replay Me! uses SQLite-a widely used open source and self-contained transactional SQL database engine-to store all the metadata associated with each video recording. SQLite is not widely used in Greenstone yet as this system has been added recently to the digital library software and it is still in the experimental stages of development. This is why, for this project, some optimisations were carried out in order to improve the time required to update or rebuild a collection using this database system.

In fact, Greenstone has the ability to update the metadata of a live collection without the need to initiate the rebuild process. This is a useful feature to let the viewers directly edit existing, or add new metadata from the web interface. On closer inspection, the implementation of this part of Greenstone for an SQLite database was realised with a simple, but disk intensive, operation. This was found to be too slow for Replay Me!, and therefore the decision was made to optimise its performance.

The first step was to make sure that each update to the SQL database was done as a single transaction which resulted in a significant speed improvement during metadata update (reducing the time taken to milliseconds instead seconds to update a single field). In Replay Me!, unlike in traditional digital libraries, the collection rebuilding process is constantly used since it is launched as soon as a new TV show has finished recording, resulting in a rapid growth of the database file. Therefore, optimising the speed of the overall building process was a key element to consider. In this regard, further experimental optimisations were implemented into Greenstone's SQLite management system, involving the use of RAM instead of the HDD for transaction journals, and also the use of asynchronous disk writings to the main database file. The speed gain is about 50 times faster than without these improvements. This enhancement was done at the price of a higher chance of database corruption in case the computer 
loses power before the data is written to disk. This is of minimal concern in Replay Me! since the building process can be started again, once the computer reboots.

An existing capability in Greenstone when configured to use SQLite is to be able to sort items in the digital library alphabetically. Of course, fundamentally, a relational database can provide more sophisticated functionality than this. For Replay Me!, Greenstone's search capability was extended by tapping into this underlying functionality. This new work enables, for instance, a user in Greenstone to search or filter recordings by date, by time or even by length. Moreover, operators such as "<" and ">" can now be used to create more interesting queries. It also allows the MediaPortal Plugin, detailed in Chapter 4, to access the information contained in the Greenstone metadata database such as the actual date of import of each recording, in order to calculate the date of expiration for automatic recording deletion.

To make the digital video library accessible to the users, the browsing structure is organised alphabetically using TV show titles, and then (for identical shows) the recordings are presented with the most recent ones at the top. An index on each metadata field (title, description, date, time, channel, length, user category, etc.) is created allowing users to search and filter recordings with any combination of available metadata. 


\subsection{Accessing and managing the digital video library}

Greenstone automatically creates a web interface to let users access the video digital library from their web browser. For Replay Me! a dedicated theme for the project was designed to create a visual identity-as pictured in Figure 5.2-different from the default look. This new theme also is the foundation of many new features, specific to this project, which allows users to directly manage the content of the digital video library from the same interface used to access and play the content. In this section more detail on the browsing interface, the searching features, the embedded video streaming player, among other content management options are provided.

\section{(1)}

1P. Location Location Location (45m 2s) 2011 12:00:00 This video has been marked to be deleted very soon.

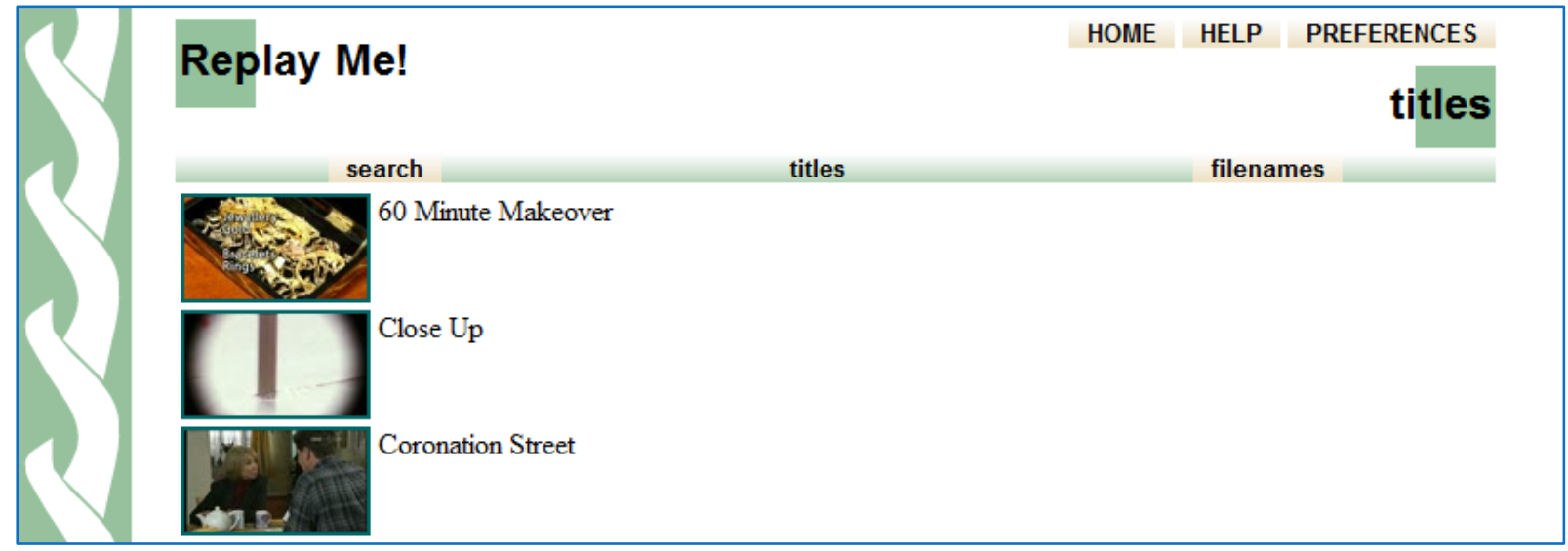

Figure 5.2

"The new Replay Me! interface (top) versus the previous default interface (bottom)" 


\subsubsection{The browsing interface}

There are two ways of finding videos in the Replay Me! video digital library. Users can browse the video collection by clicking on the "browse" button from the main navigation bar. An alphabetic list with the first letter (or number) or each show will then appear. This list is dynamic and it is refreshed each time a new recording enters the digital video library. Once the user clicks on a letter, then a list of TV shows grouped by title starting with the letter selected is displayed with a closed green folder icon-Figure 5.3. The user can click on the TV show title or on the preceding green folder icon to see more details about each video contained in that group-Figure 5.4. From this point on, the user can go back to the list of recorded shows if needed by clicking the open green folder icon; this will close the previously opened group and go back to the screen pictured in Figure 5.3.

To read the description of a specific video gathered from the EPG, the user simply hovers the cursor over the relevant thumbnail. This displays a tooltip with a written description and also automatically displays a quick visual preview of the recording-Figure 5.5. This quick preview is generated at runtime from the associated images extracted during the importing processes detailed in Section 5.1. In the list pictured in Figure 5.4, some additional information about each recording is also displayed:

- The date of original recording;

- The channel;

- The video duration;

- The remaining time before automatic deletion;

- "HD” symbol if the video was broadcasted in native or up-scaled definition;

- "CC" symbol if the video features closed captioning/subtitles;

- "MA" symbol if the video features multiple audio tracks.

Playing a recording is only one click away! To reveal the video player, the user clicks on the video title or thumbnail and the video player will automatically start streaming the selected TV show in full screen. 


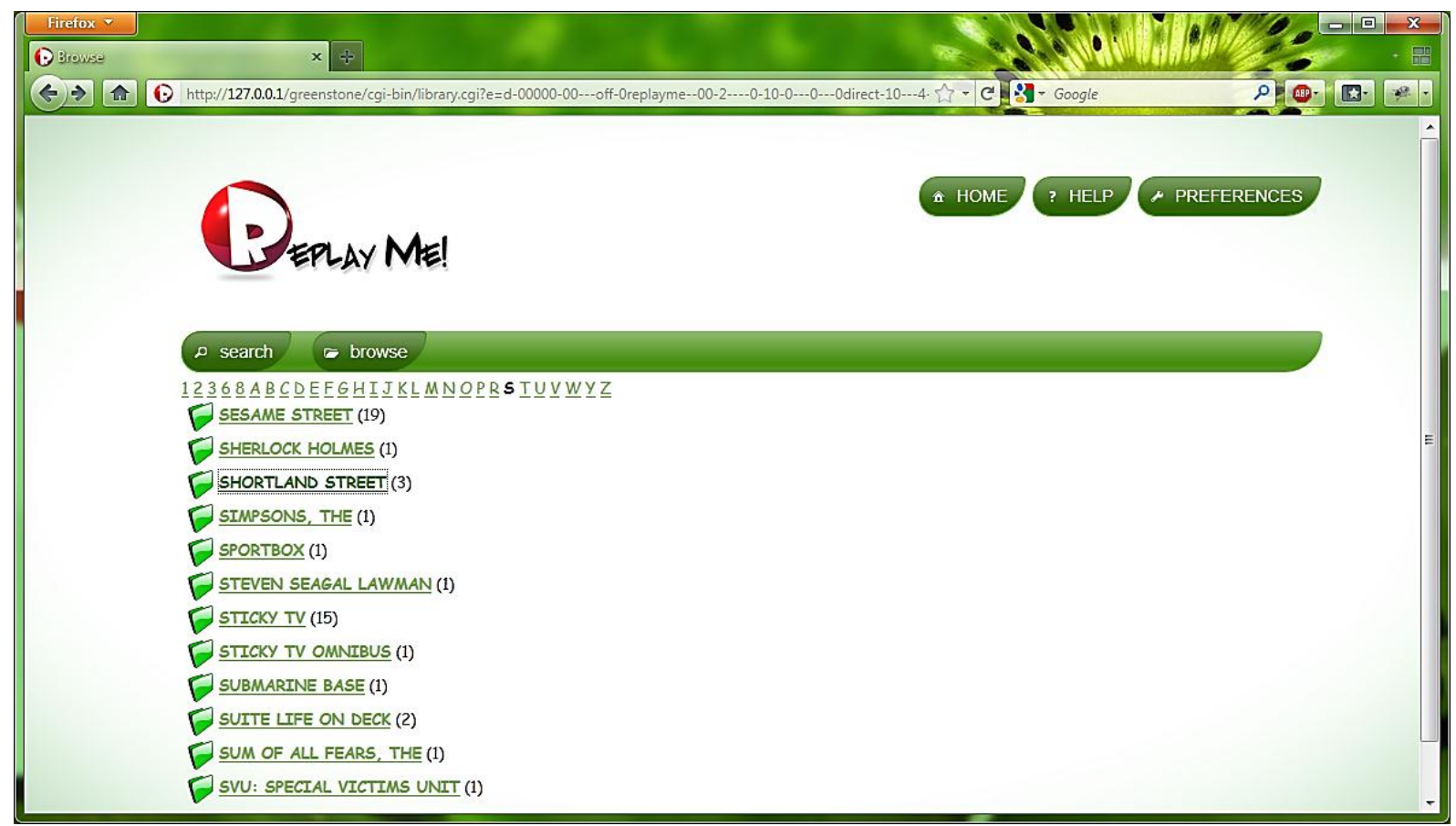

Figure 5.3

"Replay Me! web interface-browsing shows starting with the letter S"

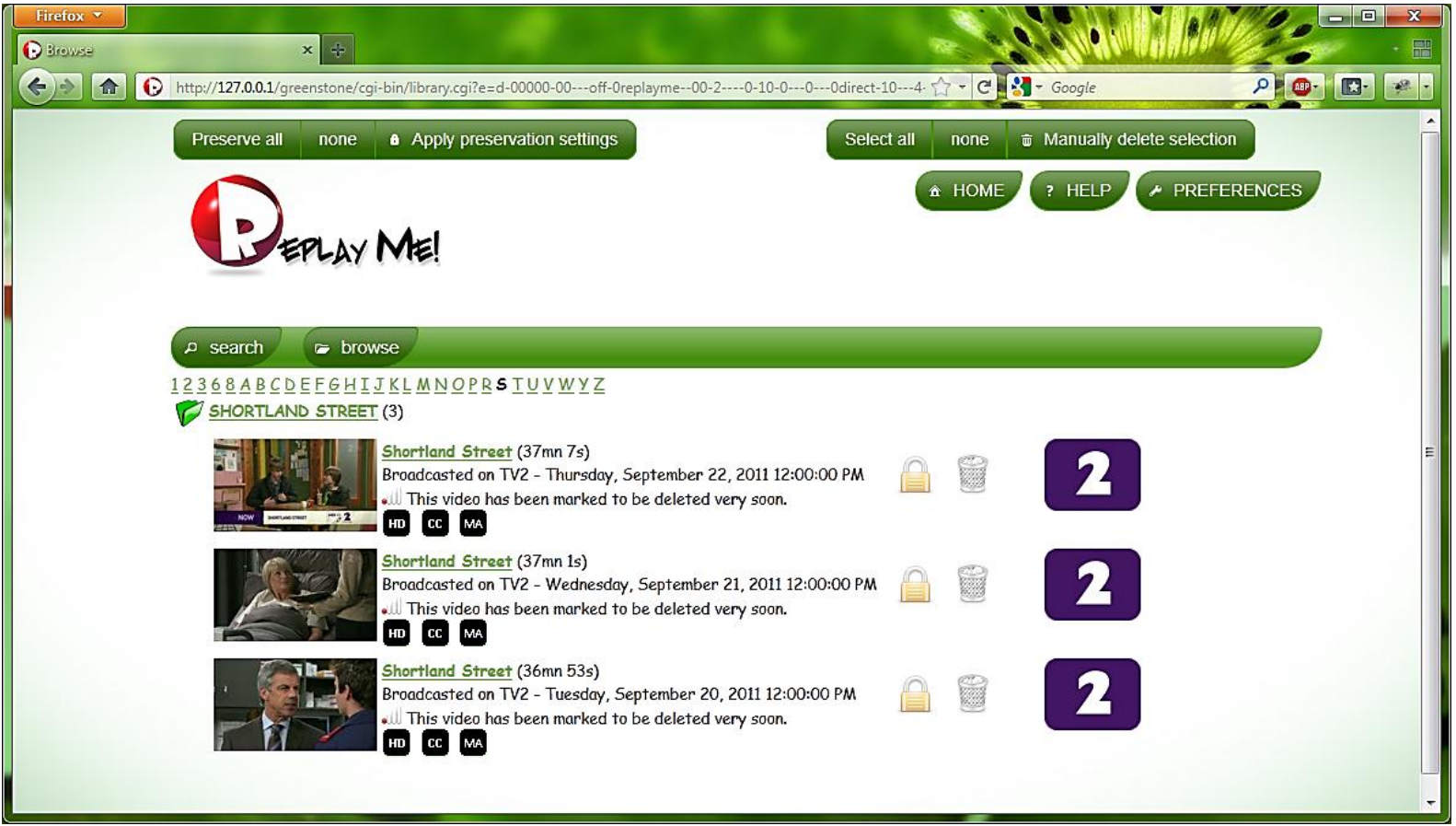

Figure 5.4

"Replay Me! web interface-browsing shows contained in the 'Shortland Street' folder" 


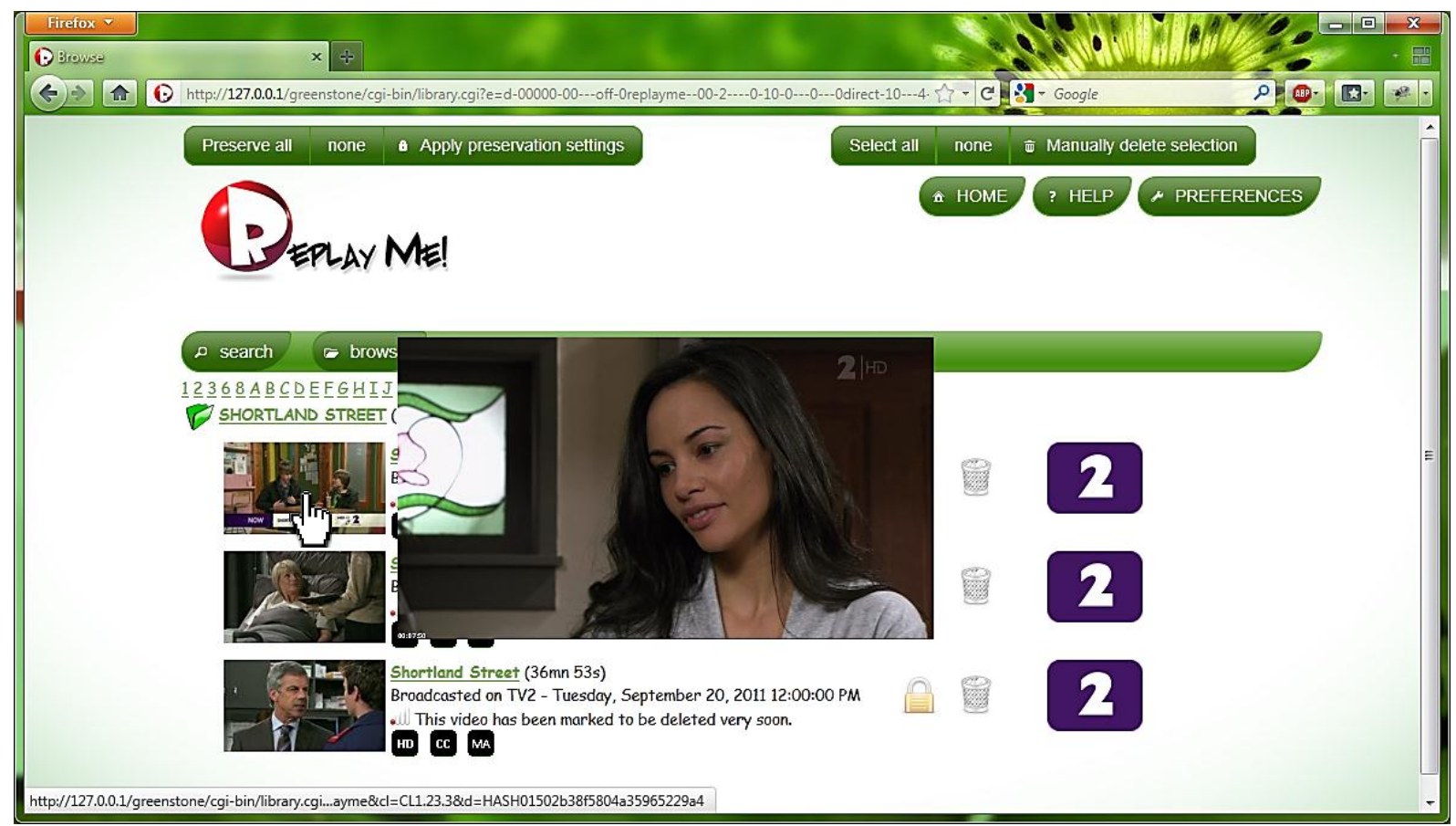

Figure 5.5

"Replay Me! web interface-fast preview of the first video from the list" 


\subsubsection{The searching features}

The content from Replay Me! can also be accessed using the search function by clicking on the "search" button from the main navigation bar. This opens a form that facilitates the creation of complex queries by using drop down menus, dynamic calendars, icons, and checkboxes.

It is possible to search for recordings that contain or start with specific words in their title or description. For example by typing "csi" in the titles field, the system will display all the available episodes of the CSI show franchise (e.g., CSI New York, CSI Miami, etc.) from all the recorded channels. Users may also choose to restrict their search on other parameters such as a specific date and/or time, channel, category, video duration, or user rating. The queries that are used on a regular basis can also be bookmarked on the user's web browser to facilitate further similar queries.

The search function also enables users to look for video recordings without having to know the title in advance. In fact, this search system is designed to easily create queries such as "Give me all the recordings that have been aired each evening with a duration of at least one hour and half from all the available channels." This type of query is likely to display all the movies broadcasted during the week (if the video retention time is set to 7 days) from all the channels-as pictured in Figure 5.6. In addition to the textual description, the fast video preview directly available in the result list can also help to choose the movie to watch since actors and other key elements of a movie, even at thumbnail size, are usually recognisable.

Users can also sort the results by date of broadcasting, title or channel by using the dropdown menu at the bottom right of the search form. Likewise they can display only the videos that have been prevented against automatic deletion by using a check box.

The result list is similar to the list of recordings in the browsing interface with the same functions such as one-click video playback, as pictured in Figure 5.7. 


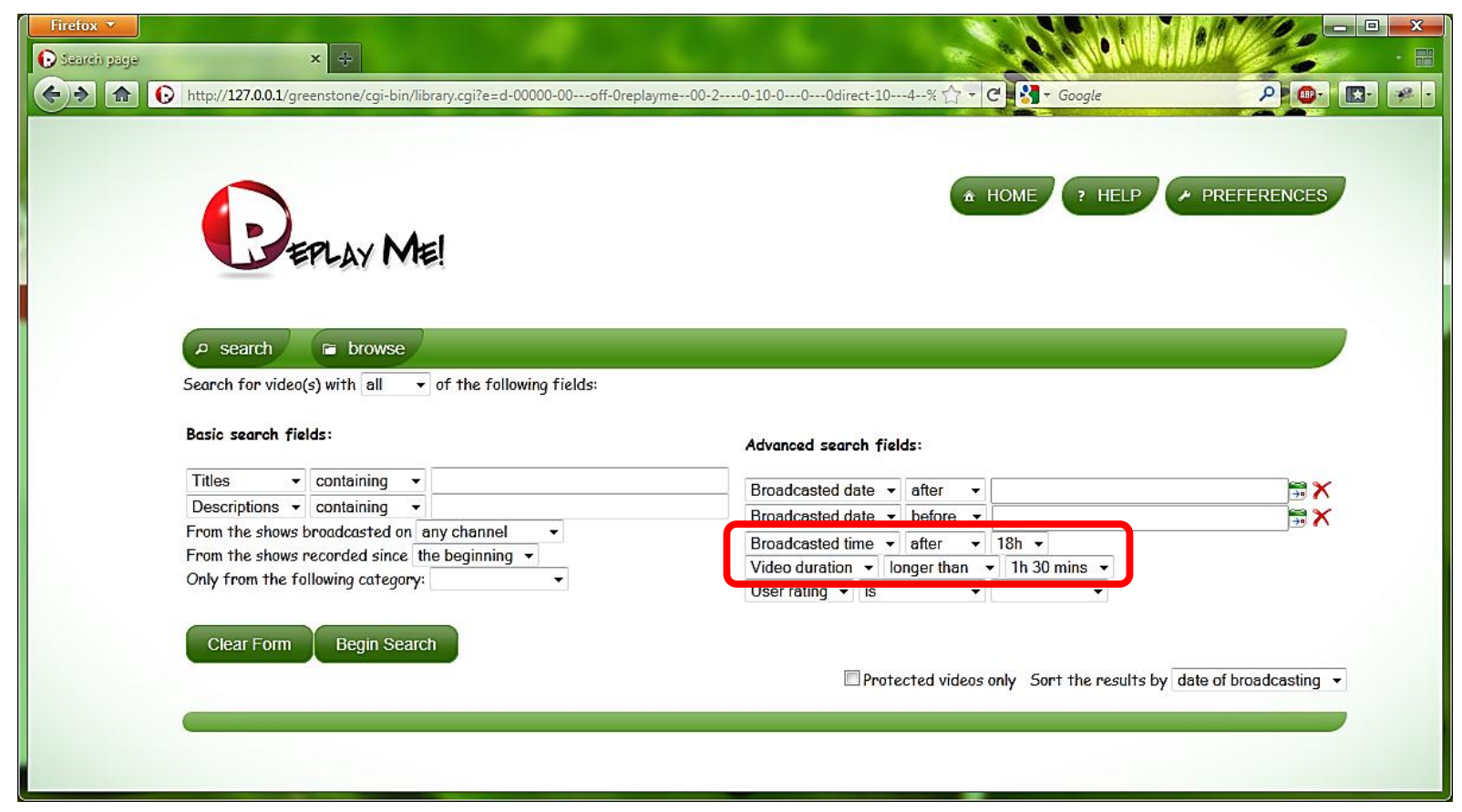

Figure 5.6

"Replay Me! web interface-searching for evening shows longer than $1 \mathrm{~h} 30$ "

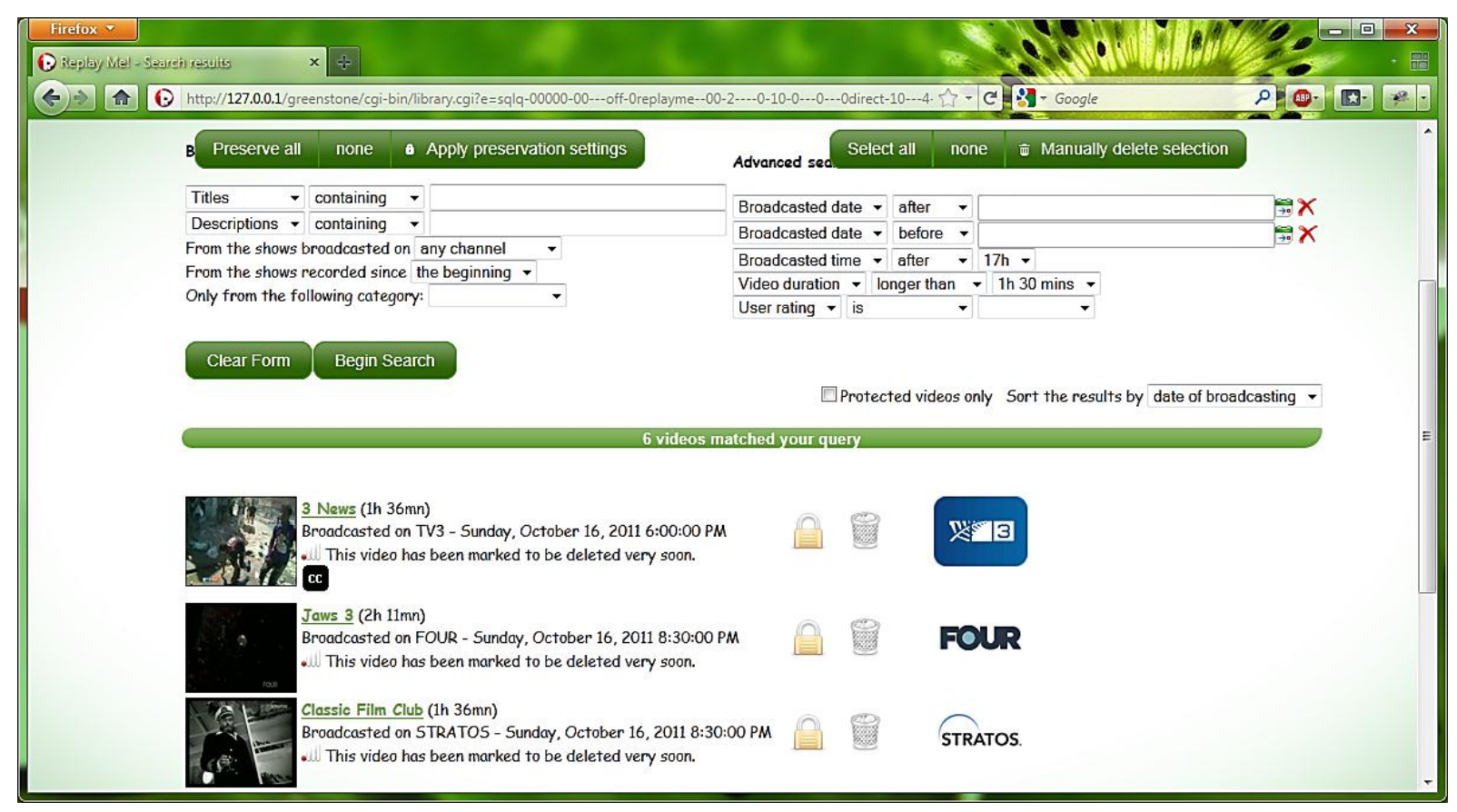

Figure 5.7

"Replay Me! web interface—results from a search query" 


\subsubsection{The embedded video streaming player}

Replay Me! embeds a video player that is based on VLC media player. This enables the playback of videos in any format directly from the web browser. At the time of writing solutions like Adobe Flash and current implementations of HTML5 video/audio decoding are not yet compatible with transport streams that are recorded from digital TV tuners. Unlike Adobe Flash Player, the VLC media player plugin for web browsers does not provide any interface for video playback-everything is done via an API which gives total freedom to developers to develop their own video player graphical interface. Therefore, a video player has been fully implemented in JavaScript, compatible with all the major browsers on the market, with all the options that are relevant to this project. This video player is versatile and can be reused in Greenstone for other digital video collections.

The video player can be launched by clicking on any thumbnail or on the title of a TV recording. This will have the effect to dim the background and open the player in a subwindow, the recording will then start playing in full screen automatically.

At the bottom of the player window, a slider can be used to seek directly into a specific part of the video-as pictured in Figure 5.8. Below the slider, a toolbar of buttons can be used to:

- play/pause,

- seek backward in 15 secs increment,

- seek forward in 1 min increment,

- toggle mute the sound,

- toggle fullscreen,

- enable/disable/change the subtitles (if available on the recording),

- change the sound track (if available on the recording),

- display additional information (title, description, channel) about the recording.

Clicking on the white cross at the top right of the player will stop and close the video player sub-window, and come back to the main video page described in more details in the next section. When closed, Replay Me! saves the current position in the video to be resumed on next playback if the user decides to watch the rest of a recording later. 
Additionally, the main features of the player can also be controlled using external input devices (keyboard or media remotes). During playback the LEFT and RIGHT ARROW keys can be used to seek backward and forward in the video, the ENTER key can be used to toggle between windowed and full screen mode. If the user has a keyboard equipped with media keys then the play/pause, stop and mute buttons will also control the Replay Me! Player. Furthermore, to facilitate navigation and playback and provide an experience similar to the television, Replay Me! is also designed to work with generic media remotes such as the example pictured in Figure 5.9.

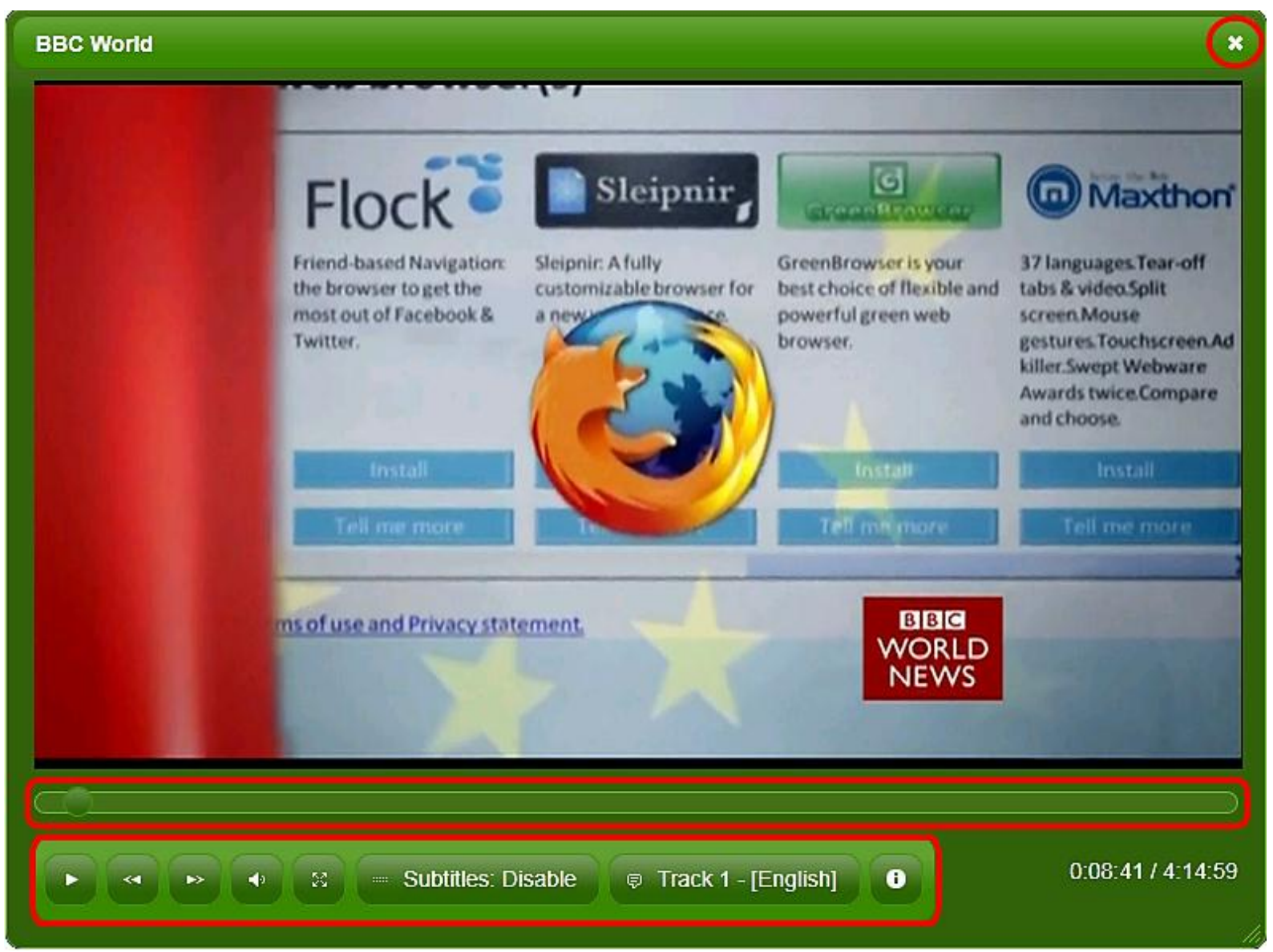

Figure 5.8

"Replay Me! web interface-Video player Interface" 


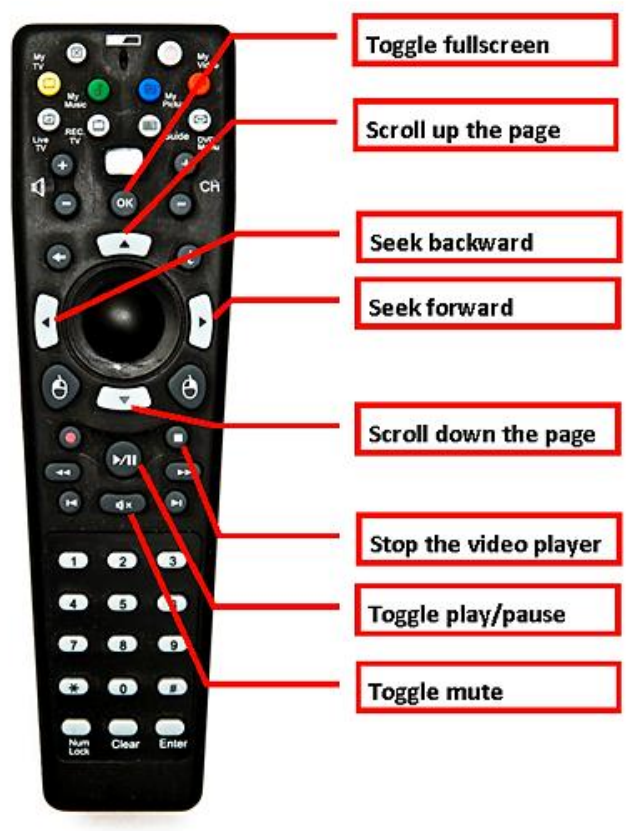

Figure 5.9

"Replay Me! web interface-Multimedia remote control usage" 


\subsubsection{Adding user-edited metadata and extra features}

Once the video player has stopped and closed, the video recording page is then displayed. This page is designed to give the opportunity to the user to add extra information about the current video that has been played-such as personal notes, a rating and a category as pictured in Figure 5.10.

To facilitate this process, adding or updating this extra metadata is usually done in a single click:

- The rating is done by clicking on the stars icon;

- Personal notes can by directly typed into the corresponding text field and pressing the ENTER key or the apply button updates the digital library;

- A category can be assigned to the current video by clicking on one of the items from the list provided in the category toolbar. This information is saved or updated and can later be used, for instance when searching the video library.

From this page, the playback can be reinitiated by clicking on the screen-view image or the "Play Me Now!" button. Additional tabs located at the top of the page provide access to further features. The "Video download" tab allows the downloading of the original video file in order to archive it on an external storage device. A download time estimators is also provided to give an idea of the time required to transfer the file given the network utilised to access the digital video library-Figure 5.11. A final tab called "Additional information," provides a summary of the technical details for the current video recording; this information can be useful for playback on other devices. 


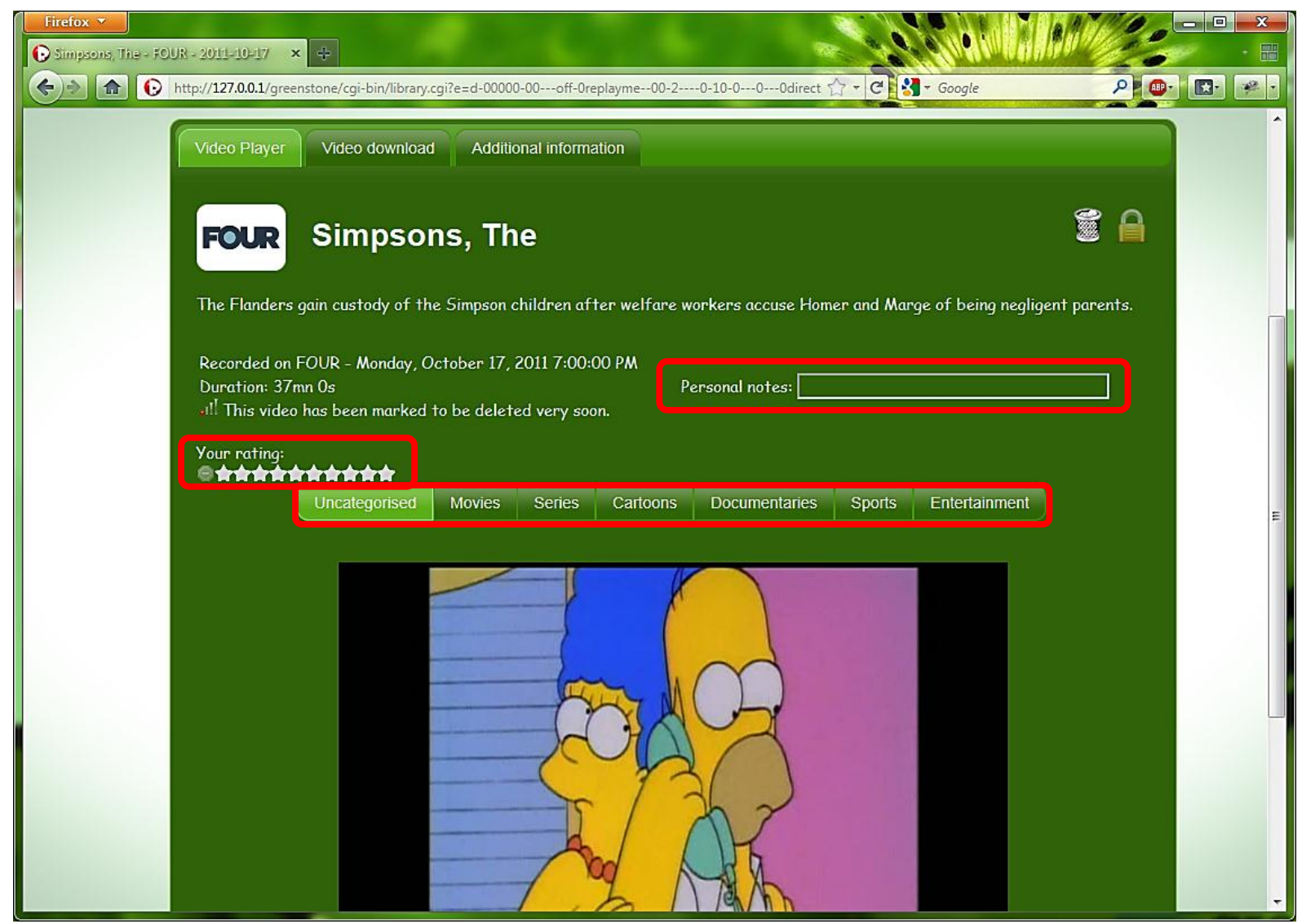

Figure 5.10

“Replay Me! web interface-Video Player tab"

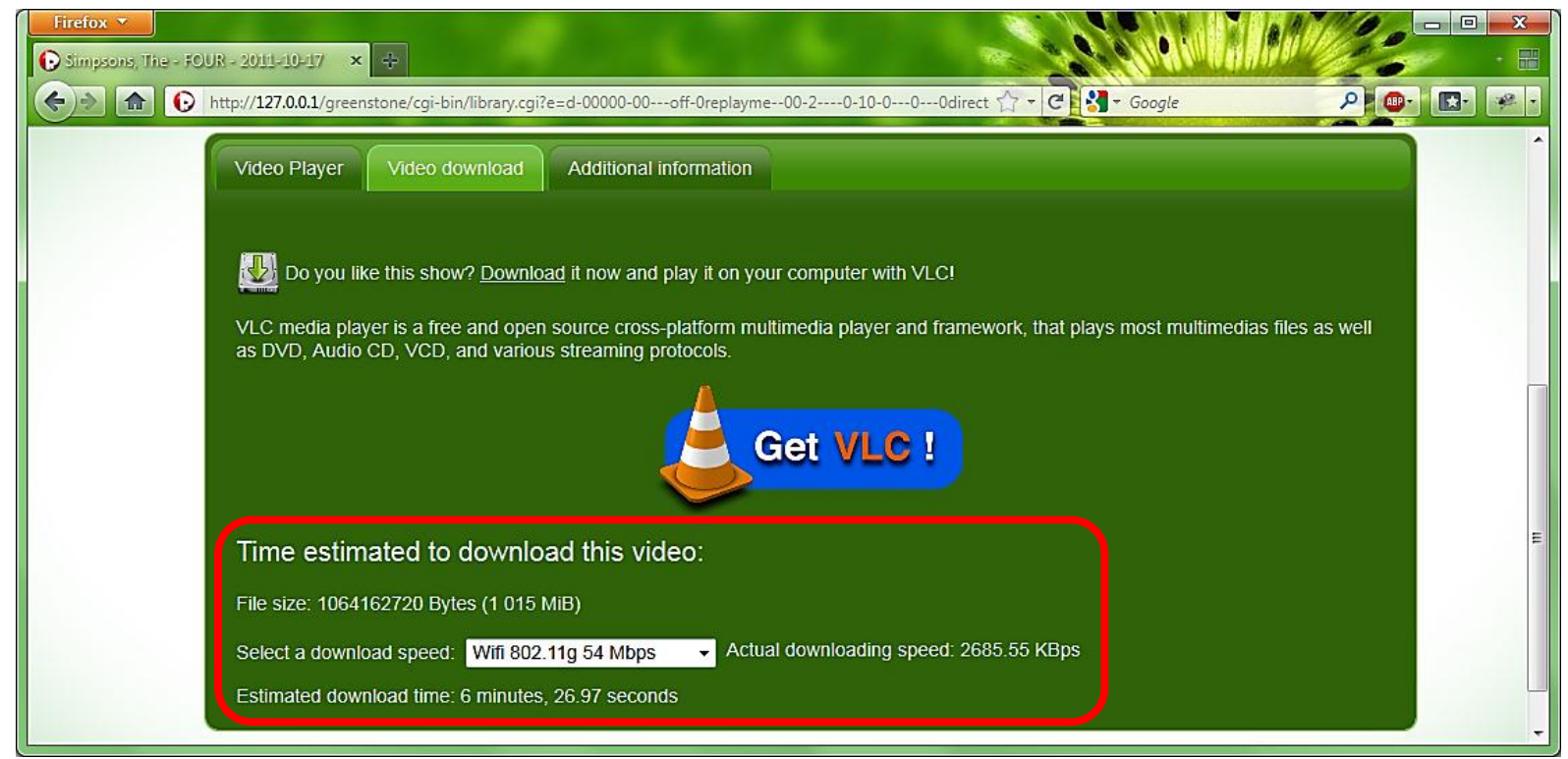

Figure 5.11

"Replay Me! web interface-Video Download tab" 


\subsubsection{Content management functionalities}

The TV recordings can be protected against automatic deletion or manually deleted from the video library directly from the web interface. During browsing and searching, a padlock icon and a trash can are displayed for each video recording. They are controlled by clicking on them; doing so will switch between semi-transparent/opaque to display a deactivated/activated state-as pictured in Figure 5.12. In this configuration, the user can mark more than one recording to be protected or deleted using these icons. Since the list can contain many recordings, a toolbar that always stays at the top of the screen has buttons to help multiple selections for keeping or deleting recordings (Figure 5.13). Then, to apply the keep or delete function for one or more videos in one go, only a click on the appropriate button from the toolbar in Figure 5.13 is required.

From the video page which is displayed after closing the video player, there are also two icons (padlock and trash can) at the top right of the page (Figure 5.14). Only this time because they are directly associated with the current recording, clicking on either icon will instantly apply the desired setting to the system. Delete-in time honoured fashion-results in a confirmation popup.

Once a change is applied, the page will automatically refresh itself if required to display the changes. 


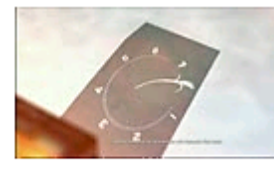

One News At 4.30 ( $30 \mathrm{mn} 4 \mathrm{~s})$

Broadcasted on TV ONE - Wednesday, July 13, 2011 4:30:00 PM

ell This video will be marked for deletion in less than three days.

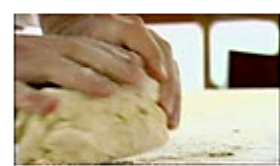

Masterchef Australia (1h $9 \mathrm{mn}$ )

Broadcasted on TV ONE - Wednesday, July 13, 2011 4:55:00 PM

will This video will be marked for deletion in less than three days.

\section{HD MA}

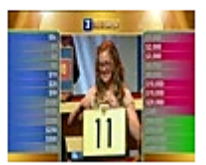

Deal Or No Deal ( $34 \mathrm{mn} 47 \mathrm{~s}$ )

Broadcasted on PRIME - Wednesday, July 13, 2011 5:00:00 PM

ell This video will be marked for deletion in less than three days.

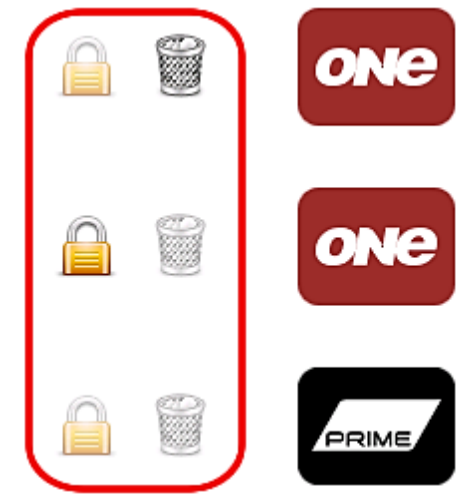

Figure 5.12

"Replay Me! web interface—padlock and trash can icons from the video list"

Figure 5.13

"Replay Me! web interface-keep and/or delete videos in batch"

Figure 5.14

"Replay Me! web interface—padlock and trash can icons from the video page" 


\section{HCI considerations and user study}

With a fully functional prototype of Replay Me! we are now in a position to evaluate it. This chapter starts by detailing the usability design practices that were followed. Then the design and goals of the user study that has been carried out are explained. Lastly, the analysed results are summarised.

\subsection{Replay Me!'s interfaces and HCI}

Usability is a key factor in designing interfaces; the idea is to make them easy to learn, efficient to use, easy to remember, aesthetically pleasing and to help to reduce human error. One of the methods to improve usability from the design stage is called heuristic evaluation which is a technique that has been popularised by Nielsen (Nielsen, 1993).

Some of Nielsen's general principles for designing better user interfaces that have been followed in Replay Me! are summarised below:

\section{- Visibility of system status}

$\checkmark$ Users can sort the results from a search query by using a drop down menu; the list of videos is then automatically updated to match the sorting option selected.

$\checkmark$ During a live metadata update or recording manual deletion from the web interface, an animated icon and/or a popup window explaining that the changes are being processed by the digital library is presented to the user (Figure 6.1).

$\checkmark$ While the Media Portal configuration plugin is testing the channels to discover if they can be recorded concurrently depending on the hardware configuration, a progress bar and a message is displayed at the bottom of the panel (Figure 4.1).

$\checkmark$ In RM's Media Portal Plugin, the "Activity log" panel displays the output from Greenstone's internal workings which can be useful for power users. 


\section{- Match between the system and the real world}

$\checkmark$ On the search page, the vocabulary used is user oriented. For instance, to quickly select a date range it is not system oriented like "date <= 15/06/2011" but instead we can "search for video(s) from the shows recorded since the beginning/ yesterday/last week" (Figure 5.6).

$\checkmark$ Similarly it is possible to select precise date ranges without having to deal with dates in numeric format such as 11/10/2011 since the system only displays dates in the long format e.g. Tuesday, October 11, 2011 (Figure $6.2)$.

\section{- User control and freedom}

$\checkmark$ When choosing a specific date range for searching the video digital library, users can quickly cancel their selection by clicking on a red cross icon (Figure 6.2).

$\checkmark$ Before actually deleting anything from the digital library, the user is presented with a confirmation popup that allows the cancelling of the operation (Figure 6.3).

$\checkmark$ Since the system is accessible from any web browser, users are free to go back to the previous page at any time, even if the displayed content is dynamic.

$\checkmark$ A button called "Clear Form" on the search page allows users to start a new query at any time. 


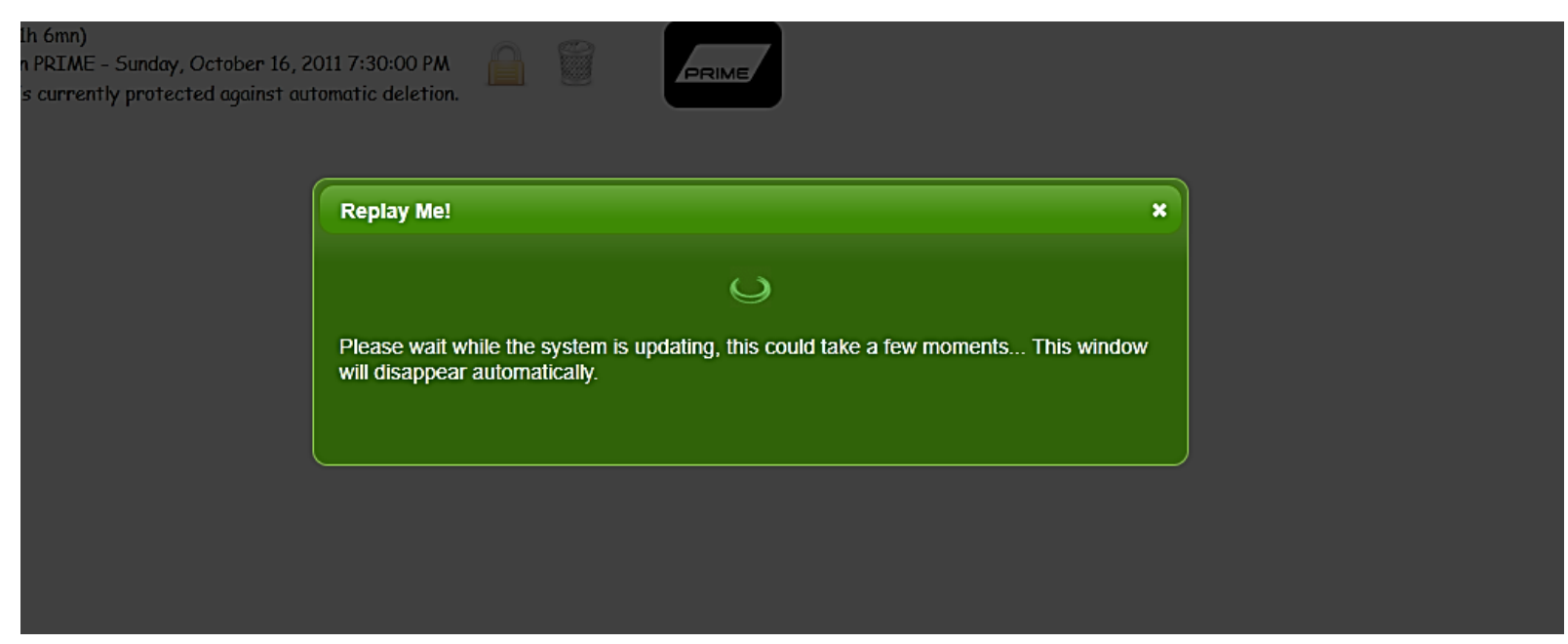

Figure 6.1

"Replay Me! web interface-waiting dialog during updates to the digital library"

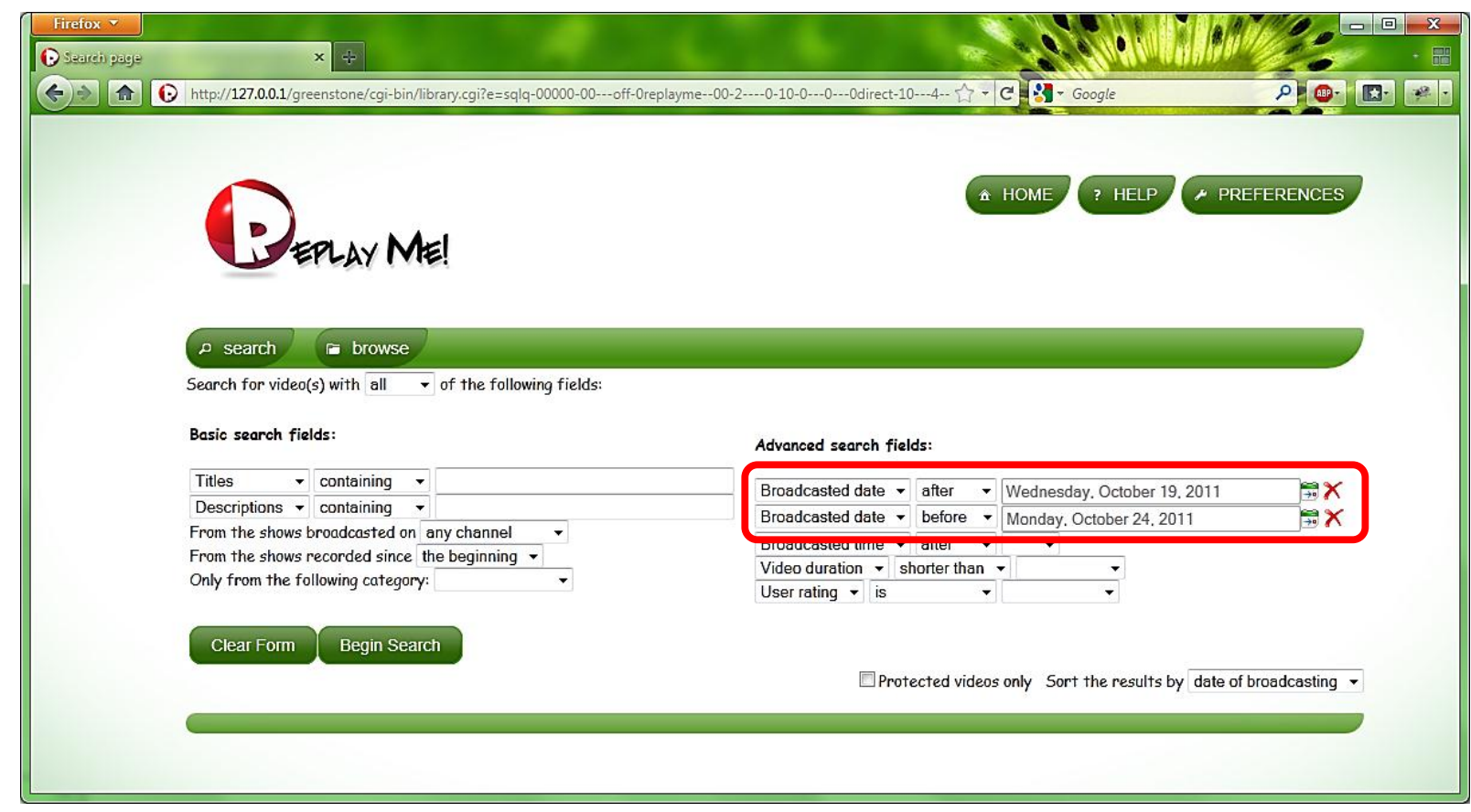

Figure 6.2

"Replay Me! web interface-long dates displayed after selection using a calendar" 


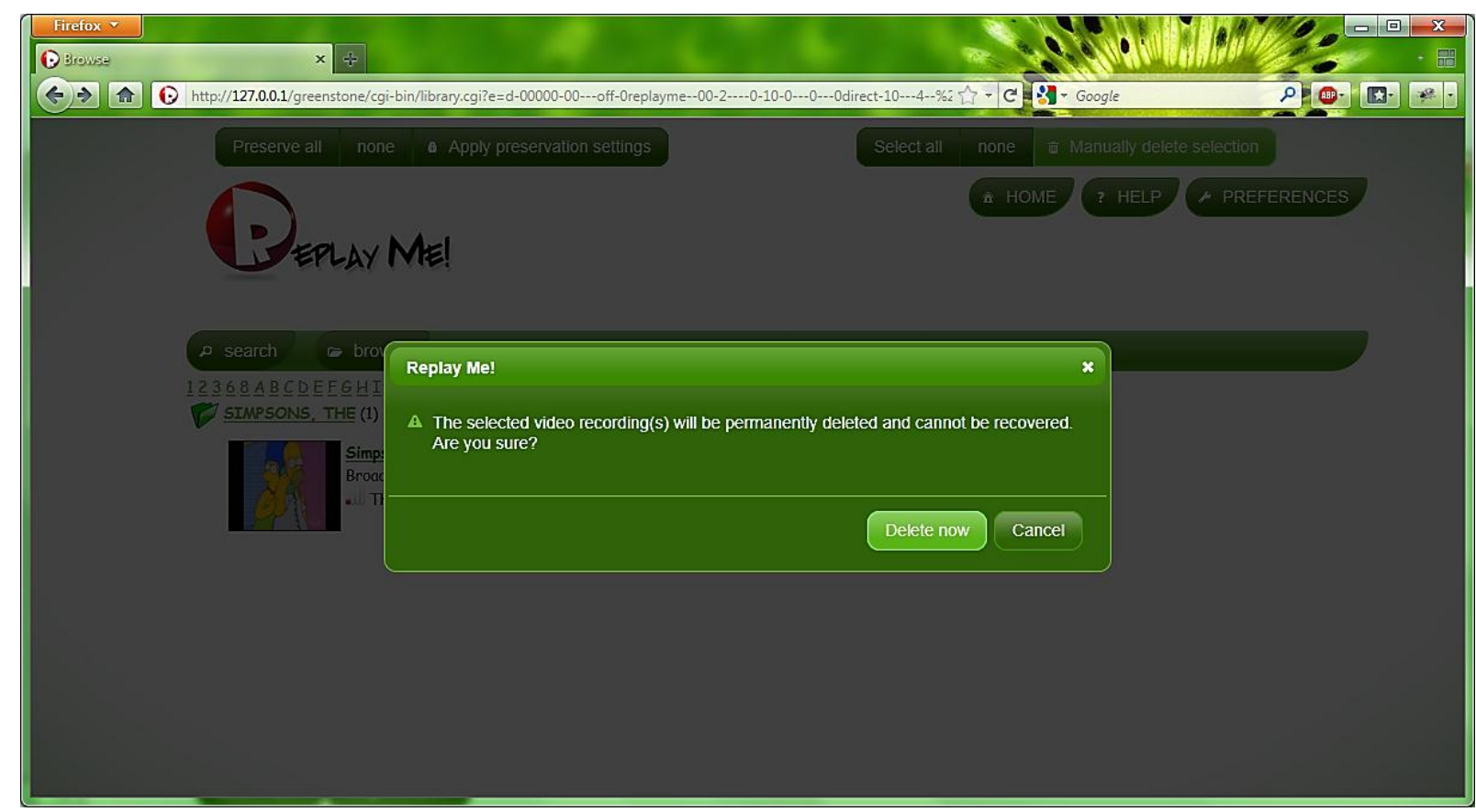

Figure 6.3

"Replay Me! web interface-Deletion confirmation dialog"

- Consistency and standards

$\checkmark$ All the widgets used to create the plugin or the web interfaces follow the platform's conventions in their look and feel, therefore only a minimal learning phase is required.

\section{- Error prevention}

$\checkmark$ Most of the search functionalities from the web interface are activated using drop down menus, check boxes, and other dynamic widgets to eliminate possible human errors that could prevent the system from executing a query.

$\checkmark$ When selecting a precise date range, the text field that indicates the selected date in long version is in read-only mode. This is to insure that users will only use the dynamic calendar to select or update a date, eliminating any typing errors. 
In the configuration panel of the RM Media Portal plugin, the root directory of the Greenstone installation can be selected via a "Find..." button. It opens a folder selection window to select the right directory. Because setting this folder is very important, it was decided to disable the direct text edit ability to this element, as this could lead to erroneous values being entered. The EPG Collector tab features a similar process.

$\checkmark$ In the RM's plugin, only one channel can be selected at a time during the selection of concurrent channel recording. This permits the system to check, for each new channel added, if the remaining ones can be also added to the list or if they need to be hidden to avoid a selection combination that is impossible to record simultaneously (Figure 4.1).

\section{- Recognition rather than recall}

$\checkmark$ Icons are widely used throughout the web interface to help users recall functionality more easily.

$\checkmark$ A calendar is displayed helping to select a date using a few clicks.

$\checkmark$ The appearance of the video list between searching and browsing is presented in exactly the same way.

\section{- Flexibility and efficiency of use}

$\checkmark$ The video player is designed to be flexible enough to be controlled directly from the keyboard and also using media keys allowing multimedia remotes to be used (Figure 5.9).

$\checkmark$ Accelerators are present: for instance it is possible to select or deselect multiple videos at once to be kept or manually deleted.

$\checkmark$ The "Collection Settings" tab in RM's plugin is for expert users, familiar with Greenstone. It allows the direct editing of the configuration file used to create the video collection. This is where one might change the way each recording is indexed and organised. Other advanced settings can be adjusted such as the recompression format, the bitrate used for the video and audio, the video dimensions, etc. 
- Aesthetic and minimalist design

$\checkmark$ During video playback or when a dialog popup appears in the web interface, the background is dimmed in order to reduce the number of distractions and lead the user's eyes to the most important elements on the page (Figure 6.4).

$\checkmark$ The theme is based on a white background and the green colour to produce a light and relaxing design.

$\checkmark$ The list of recordings during browsing or searching is not cluttered with technical data. Some information is only displayed in a graphic manner instead of text such as the small "HD" symbol under the recordings thumbnails.

\section{- Help users diagnose, and recover from errors}

$\checkmark$ If no Greenstone installation is found in the folder indicated by the user using the "Find" button as explained earlier, an appropriate error message explaining the situation and where to download Greenstone is displayed (Figure 6.5).

\section{- Help and documentation}

$\checkmark$ Complete documentation of the web system is accessible at any time using the help button at the top of every page. It contains information and screen shots to quickly learn the possibilities offered by Replay Me! and also ways to troubleshoot errors that may occur.

$\checkmark$ All the icons and other graphical elements from the web interface feature tooltips in order to explain their meaning. 


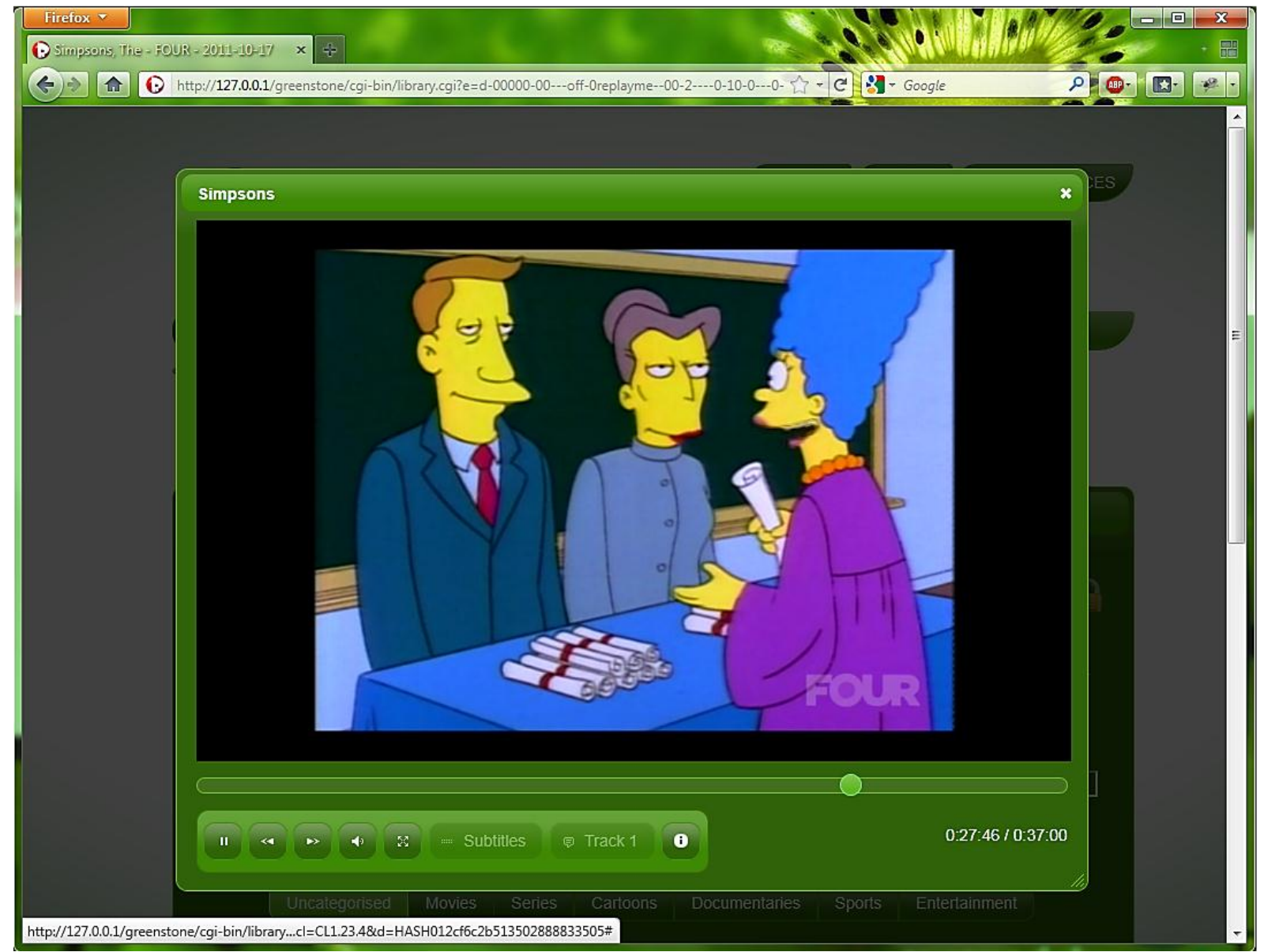

Figure 6.4

"Replay Me! web interface-Video player with dimmed background"

\begin{tabular}{l} 
Replay Me! \\
$\begin{array}{l}\text { No installation of Greenstone was found in this directory, please check } \\
\text { that you have selected the right folder. } \\
\text { You can download and install Greenstone for free from } \\
\text { www.greenstone.org }\end{array}$ \\
\hline
\end{tabular}

Figure 6.5

"Replay Me! plugin—Greenstone not detected in selected folder error message" 


\subsection{User study design and goals}

Having developed these capabilities for Replay Me!, it was possible to evaluate the user's level of satisfaction by undertaking a user study. In order to gather more realistic data, instead of asking participants to come into a university lab for an hour or so, and pretend they had, for example, "just come back from work at the end of the day, relaxing in front of their favourite TV shows", it was decided to bring Replay Me! to the participant homes, and leave the system on site for a few days.

To help sorting through the differences in people's home setups, two pilot user studies were undertaken aiming at discovering and rectifying possible issues with the protocol that could prevent the study from being carried out successfully when scaled up. Afterwards a full-scale study was achieved with the participation of 11 people from different backgrounds, with an age ranging from 20 to 65 and over.

For each user study, a house visit was made by the researcher to make sure that all the steps were taken in order to connect all the new equipment to the participant's electrical network while maximising safety. For instance, the system was not connected to an overloaded multi-plug or somewhere that could be dangerous. The equipment lent for this experiment passed all the necessary tests to be connected to the University electrical network, and therefore equally be safe to use in a home environment.

Once everything was setup, a quick test for television signal strength was carried out. If the television signal strength was adequate, the experimentation could then proceed if the participant agreed to give his/her consent for the study. Then, a password protection mechanism preventing access to inappropriate television content from any children living at the participant's address was installed.

Since only one system was available, it could not be left for too long at each participant's place since it would have reduced the number of possible participants. The trials of this study lasted for almost 12 weeks in total. 
The method applied to this user study for each participant was as follows:

- Each participant was provided with the machine allocated specifically for this project and was installed at their place of residence.

- At the start of the experiment, participants were asked to follow a simple tutorial exercise in the presence of the researcher in order to quickly familiarise themselves with the system.

- The participants were asked to use Replay Me! to watch television shows in a real life situation, exactly as when they were using their traditional television.

- The equipment was left with the participant for 3 to 7 days, but the time spent using the software was up to the participants.

- For the entire duration of the experiment, the participant's activity was automatically logged whenever they were using the system.

- Once the experiment was completed, the participant then completed an electronic questionnaire. 


\subsection{Results summary of the user study}

The full-scale user study being very similar to the initial pilot study, all the results summarised in this section are analysed from the data that has been gathered during both experiments. This effectively increased the number of participants to 13. First, general results regarding participants' satisfaction are outlined, and then a more detailed account about the practicability of each of the main features of RM is given. Additionally, recurring views and ideas made by participants during the experiments are presented.

\subsubsection{General and system satisfaction results}

Overall, people found Replay Me! useful and intuitive-in particular its key concept of having everything from all channels accessible anytime was universally liked. Specifically, $62 \%$ of participants rated the usefulness of the general system with the top mark of $5 / 5,31 \%$ of participants rated RM at $4 / 5$ and $7 \%$ at $3 / 5$. This proves that there is an irrefutable interest in the technology developed for this project as most, if not all, of the participants regretted not being able to keep trying the system for a longer period of time.

Being able to record all the channels concurrently is one thing, but this will be for nothing, if users are not able to find their way to the content they wish to view. At its disposal, Replay Me! has access to the EPG in addition to the data that can be extracted from each recording and augmented by user input. Making the most of all the available information, as much functionality was added as time allowed. The user study was a great opportunity to discover which features were the most useful to our target audience.

The pie graph shown in Figure 6.6 reveals the utilisation proportion of the main features by all participants calculated from the activity logs. The fast skipping function, used to precisely skip ahead or backwards for a predefined number of seconds, is by far the most used function of all. This function alone accounts for $62 \%$ of usage, and it was frequently used by participants to skip advertisements $(1,751$ occurrences in total across all participants).We note here that it takes several clicks in the interface to skip an advert (for example, for a typical advert break 4 clicks forward for 4 minutes, and 
then a couple of clicks back at 15 seconds each to home in on the resumption of the show). This is why this category is so much higher than the other categories. The second most used function is video playback with $16 \%$; this shows that people did successfully find their way around the system to locate programs that they considered worth watching. The seeking slider bar on the video player used to directly seek into parts of a recording to find interesting parts is the third most used function accounting $9 \%$ of overall usage. Following this, browsing and searching are particularly close-they respectively account for $5 \%$ and $4 \%$ of overall usage. Pausing the video playback also accounted for the final $4 \%$ of usage.

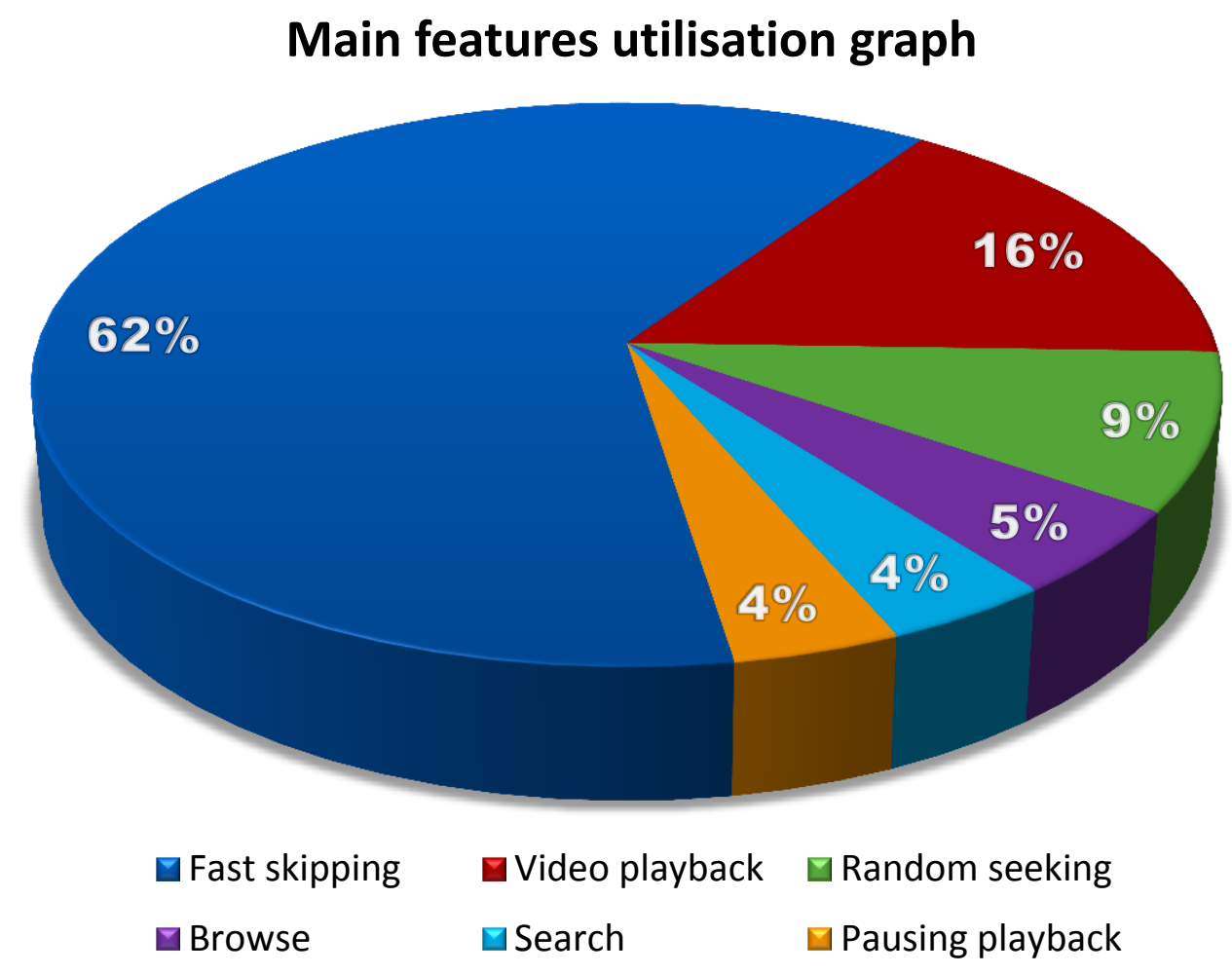

Figure 6.6

"Utilisation graph of the main features of Replay Me! by all the participants" 
While the previous results derived from the activity logs give an idea of the real usage, the user satisfaction was also measured. The bar graph computed from the average satisfaction results of all participants on a scale of 1 to 5-shown in Figure 6.7-clearly shows that the search action is as important as the browse function. Indeed, each participant had a preference on which feature to use to get access to recorded TV shows, but on the whole, browsing usage accounted for $53 \%$ while searching for $47 \%$.

The main functions appreciated by all the participants in order of preference were:

- resuming the playback of a video from the last known position,

- keeping the recorded videos until manual deletion,

- fast skipping forward and backward (usually used to skip advertisements),

- fast selection of a date range in searching (show only videos recorded since the beginning/yesterday/etc.),

- quick sorting of the search results to only display the recordings that have been manually kept into the system,

- sorting options during search such as by channel, by date, or alphabetically,

- and placing video recordings into predefined categories.

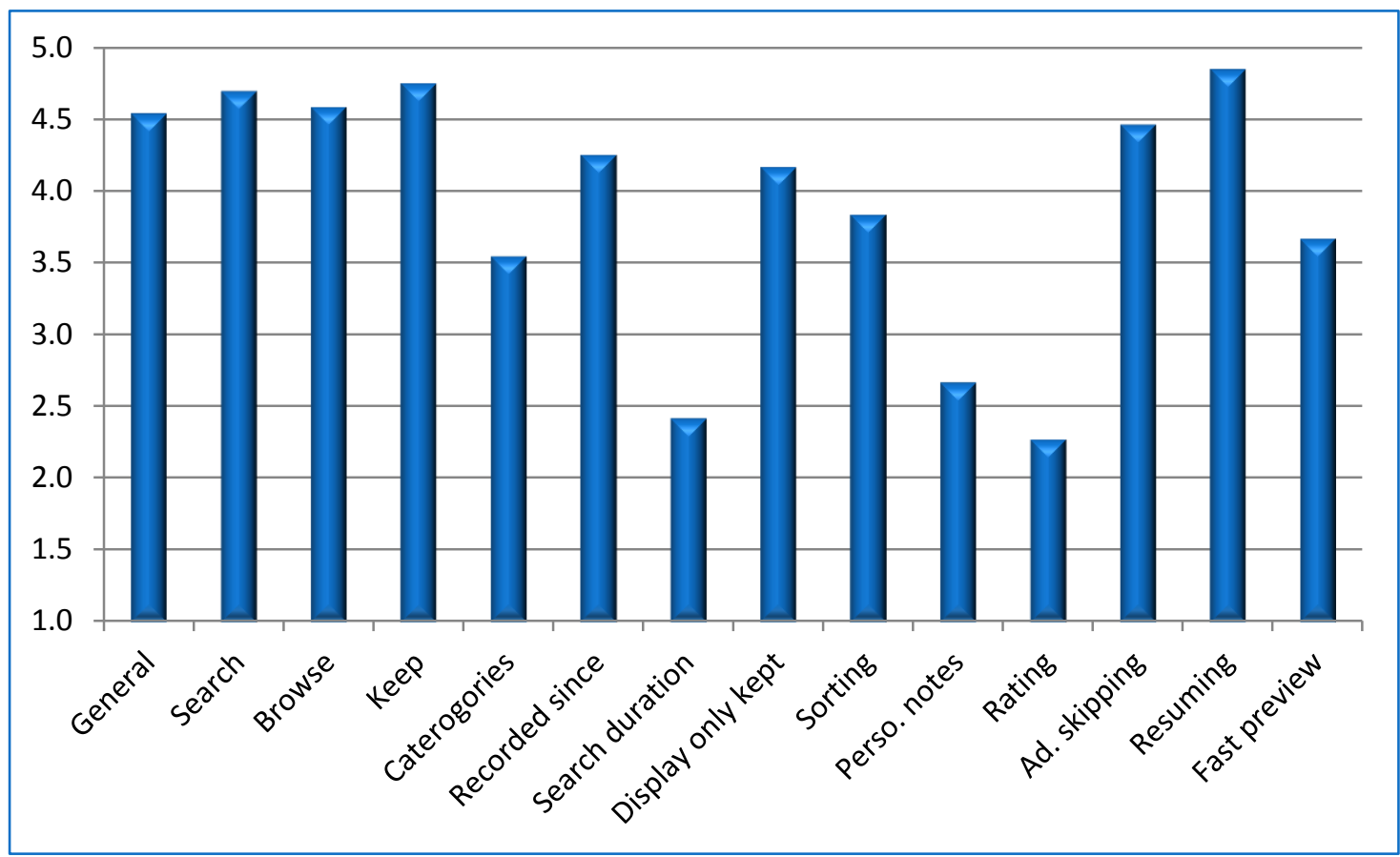

Figure 6.7

"Satisfaction results graph from all the participants" 
However some other functions appeared to be less interesting to the participants:

- adding personal notes to recordings,

- search videos by duration,

- $\quad$ and setting a rating for TV recordings.

Most likely, the real situation is more complex as analysis of the global results also shows where some participants found the search by duration function really helpful to find, for instance, movies or main sports events from the digital video collection. This could be an indication that the main goal of this functionality was not clear enough to the target users, and should probably be presented in another way.

The data was segmented by age and by gender but no discernible differences were found. More distinctive was the division:

- People who did not have (prior to installing RM) the possibility to record television shows for later viewing;

- People who already has systems that supported recording shows manually or semi-manually for later playback.

As a result, these two groups of participants did not compare Replay Me! against the same system (traditional TV or HDD recorder).

While the overall trend is similar-as shown in Figure 6.8, these are also some differences:

- Traditional TV users did rate features designed to find videos significantly higher than features used to manually add extra metadata—such as personal notes or a user rating;

- HDD recorder users, on the other hand, did rate features used to manually add metadata higher, most likely because they are used to doing this with their own recording system;

- The advertisement skipping feature was better rated from the traditional TV user group than from the HDD recorder group. Since this function is more or less standard on HDD devices today, the value it adds appears to have not been perceived as so high as by the latter group. This is in spite of the fact that the 
automatic data logged during the experiments showed the same amount of usage (extremely frequent) of this function by both groups.

These differences show that the satisfaction towards the system is interrelated with the initial habits of the user. Actually, this is also reflected by the comments from participants having an HDD recorder, since (when it came to features the RM prototype lacked) most of them desired a system that was as close as possible to the system they were currently using.

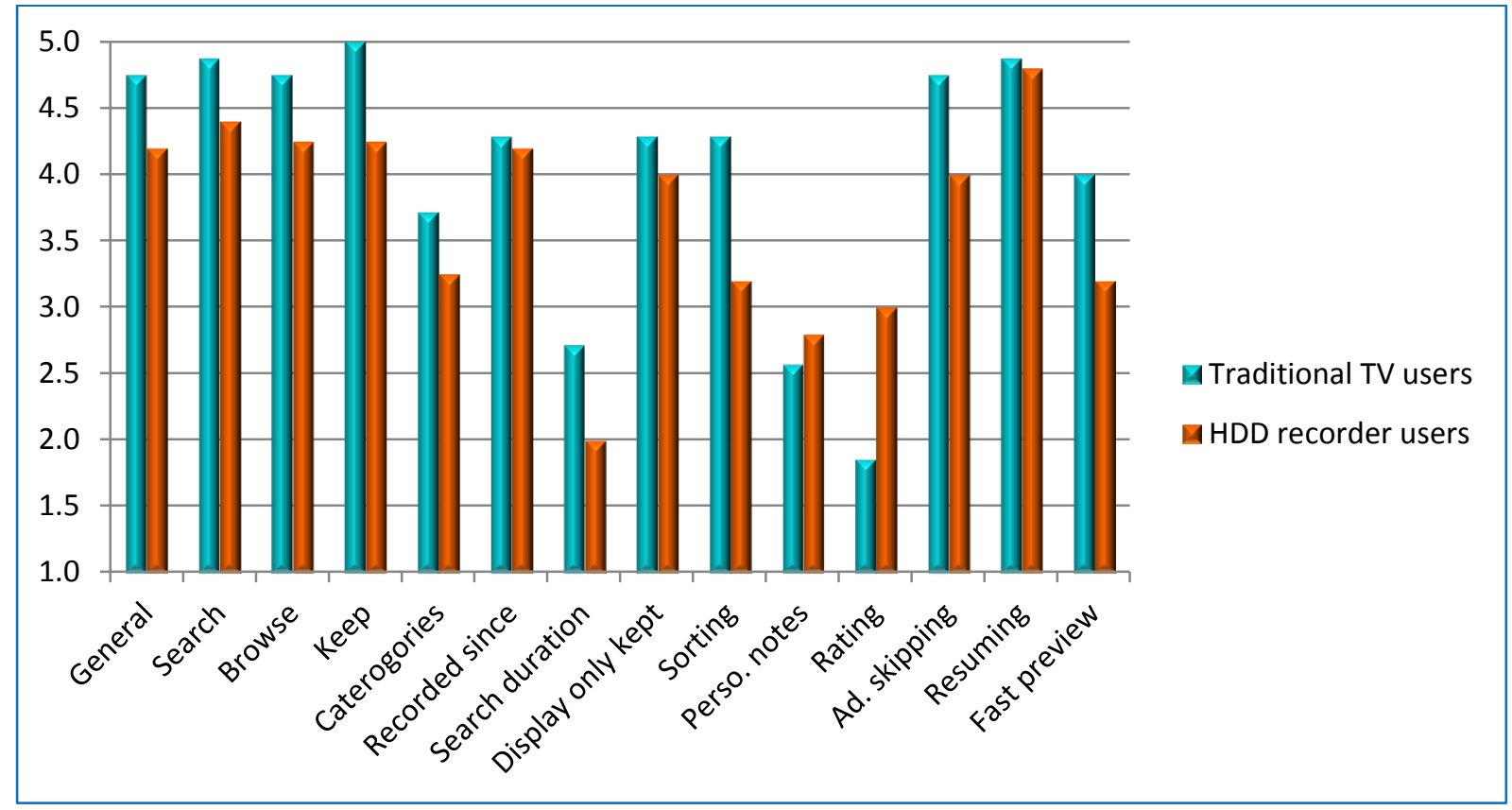

Figure 6.8

"Satisfaction rating of traditional TV users versus HDD recorder users" 


\subsubsection{Ideas and comments from the participants}

The participants had many comments on what they found annoying with their current system (simple TV or HDD recorder), the most recurrent ones are summarised below:

- Not being able to watch a program because the broadcast time is not convenient (usually too late);

- Multiple interesting shows coinciding at the same time on different channels;

- No quick view function to see if a recorded program has been already watched or not;

- Missing some recordings due to unadvertised changes of time from TV channels;

- The requirement to proactively record a show if not at home at the time of airing;

- Difficulty finding what is already recorded.

These comments show the need for a system that exonerates users from being requirement of proactively setting up recordings, while still having multiple ways of accessing the content. People definitely want the choice of the TV shows to watch in addition to the choice of timing.

At the end of the experiment, each participant shared their experience with using Replay Me!. Some of their positive comments are outlined below:

- Ability to watch interesting programs that were not normally seen due to their time of airing;

- Avoiding clashes with good programs on different channels on the same time;

- Can quickly see what good shows there are on, on all channels, to watch;

- Can watch multiple episodes in one sitting;

- The set of options provided is definitely helpful;

- Ability to look more closely at TV programs that are not usually recorded, to decide if they were worth watching;

- Watch the news programs from any channels at a convenient time since this is a type of show that participants do not usually record;

- Being able to watch something a couple days later if someone recommends/talks about a programme that was missed;

- Unlike TV on demand services where there is a delay of 24 hours before shows are accessible, RM has them in the library nearly instantly after broadcasting; 
Some quotes from the participants are presented below:

- "I could stay longer at work without missing a program";

- "I do not have to think which shows I have to record since everything is already recorded";

- "RM records all the channels every day whereas my system only record what I selected";

- "I also found lots of other interesting things to watch that I hadn't known were there";

- "I can come home and review what has played during the day instead of having to plan in advance";

- "I can watch things I haven't explicitly recorded. This is the best feature";

- "It is a far superior system with the freedom traditional TV does not have";

- "It would soon take over my normal TV viewing";

- "This system is well adapted to people like us who have a busy life";

- "I would be interested in purchasing such as system should it become available on the market".

These comments clearly show that people are interested in what the technology can provide to simplify their life and enhance their entertainment. Replay Me! is a good example since it gives the choice to the user of the TV shows to watch without having the requirement to plan the recordings in advance. Moreover, RM allowed participants to discover new programmes, which they did not discover even with the use of their current recording systems.

Interestingly, having an increased choice of program to watch does not mean people watch more. The results for these experiments do not show any significant difference in the time spent to watch television with or without using this system. However there is definitely an interest for the technology since most participants themselves inquired as to the possible commercialisation of this system. All in all, it is clear from these comments, that Replay Me! gives practical solutions to most of the issues that frustrate people with their current systems. 
The participants, being excited about the testing of this system, also wanted to make some new feature suggestions that could further improve this system. A summary of these are:

a) Being able to watch a program from the beginning, while it is still being recorded (Time-shifting)

b) Play lists

c) On screen video editing to remove advertisements and export to DVD

d) Browse the content per channel by time (like the TV guide)

e) Automatically keep all episodes of the same name

f) See the programs in the system before they are actually recorded

g) Quick links to the next and previous programs

h) User defined categories

i) Add an indication to the shows that have been already watched from the list

j) Predefined searches such as "recent news", "movies", etc.

k) More information about movies, actors

l) Buttons to increase or decrease the font size

While no system is perfect, these recommendations are definitely good ideas to further improve the system, even if some of them imply a constant Internet connection and sometimes a subscription to rich metadata databases. It is interesting to note that most of these features come from the system that participants already own. This suggests that people's habits, unlike technology, are persistent in the long term.

Some of the features recommended by the participants can be integrated into RM through only modest enhancements. For instance, the proposed features d) e) and j) can all be implemented by utilising pre-defined queries embedded (say) in the main navigation bar. In fact, it is already possible to browse the content by channel by issuing a search query that only displays videos broadcast by a certain channel and have it sorted by time. Similarly, predefined search queries could help people to locate the movies or news from the video library. An embedded link "latest movies" could be hardcoded to search for the latest recording lasting more than an hour and a half, for instance. Similarly, user defined search queries (used to retrieve the programs with the same name in order to keep them batched together) could be saved and periodically rerun. 
It is important to notice that all these comments are certainly interesting extra features for the system; however they do not challenge the key concepts of Replay Me!-which are the automatic recording of all channels simultaneously, and the indexation of the segmented video content into a digital video library. 


\section{Conclusion}

Today, it is clear that people still watch TV through a device dedicated to the purpose, but this popular leisure-time pursuit is evolving. A recent trend for video on demand technologies is growing rapidly. The development of television, together with the increasing amount of digital information has brought new challenges to manage multimedia content. The increasing number of available television channels creates opportunities for new systems to be developed. Consequently, a practical system that provides improved access and control of this increasing volume of broadcast material have been built.

As summarised in Chapter 2, this increasing volume of video content being digitised or born digital, has prompted the digital library research community to work on the subject. The goals of these projects range from the extraction of information contained in graphical and temporal data, to video segmentation techniques that retain entire coherent sequences. In its present state, Replay Me! segments videos according to the information gathered from the EPG. However, it would be interesting to also create chapters in these video segments using the advanced image processing from the Informedia Video Digital Library project, should these technologies be released as open source software in the future.

Some commercial products are already on the market, but the only systems that record many channels concurrently to date are aimed at large organisations, governmental agencies or educational institutions. These solutions use powerful enterprise grade hardware which is not financially accessible to the wider public. That said, the equipment used in enterprises today will become the standard of tomorrow's residential equipment. This can play out in various way to the advantage of Replay Me!. For instance, the size of the cost of the equipment can be reduced and/or the numbers of channels recorded or the length of the window captured increased.

Other commercialised products are aimed at the residential market but intentionally restrict some functionality because of commercial factors involved with each offering. This is where Replay Me! differs from the current commercial offering-this system was developed with the aim of using the raw technology to solve a maximum of the challenges that digital television presents today. 
In summary, the aim of this project has been achieved since the implementation and user testing of Replay Me! permitted us to look beyond what is currently available on the market today and to explore more fully the potential to what the raw technology can provide.

The Replay Me! prototype is a system that features concurrent recording of all TV channels with automated organisation of continuous TV streams, while still allowing manual management of collected TV shows directly via a graphical interface accessible through any web browser. Moreover, this system allows multiple playbacks whilst still recording with full services available within home and a reduced set of services available off-site (depending on the network upstream speed available). Furthermore, this extended set of functionalities can work independently from broadband access on equipment suitable for a home environment since it is implemented using a single desktop PC with free open source software.

During the development of Replay Me!, Greenstone-the baseline digital library software used in this project-has been improved in several ways in order to comply with the requirements of the project.

The following improvements to the Greenstone software were critical to Replay Me!'s operation:

- Numeric sorting implementation;

- Multi-format JavaScript video player implementation;

- Full H.264 compatibility of the video document processing plugin.

Further improvements to the Greenstone software were not critical to Replay Me!'s operation but still provide interesting opportunities for other digital library projects:

- SQLite speed optimisations;

- Thumbnail extraction speed optimisations;

- Integration of a new algorithm (The Movie Thumbnailer) for video summary creation.

To conclude, this system has been proven to successfully free users from the schedules imposed by broadcasters and to put users back in control of the choice of the television 
programs being offered since everything is available locally, on demand, with no loss of quality.

Considering future work, it would be interesting to further develop this system along the lines of the features suggested by the participants of the user study. A natural next step would also be a solution to allow searching within the video recordings by getting access to the subtitles broadcast with some programs, as this would be particularly beneficial. This implies the usage of OCR techniques since in New Zealand the terrestrial Freeview platform uses a format based on images for broadcasting subtitles as this more naturally supports internationalisation.

In many ways the system developed in this project (surprising even to the author!) competes closely in functionality with current enterprise solutions, despite using much more modest equipment. Even with a conservative estimate, scaling of the design to record 50 digital channels concurrently would only require:

- A single PC;

- 4 dual tuners (taking in account that each MUX can broadcast up to 8 channels);

- 6 x 3TB HDD in a RAID array (to store 50 standard definition channels for a week).

This expanded system would rival SnapStream's DVR shown in Figure 2.2 since Replay Me! does not need any extra work to operate in any of the major TV zones around the world.

In terms of the planned focus to the project, the user study that has been carried out establishes that the wider public is definitely interested by the opportunities offered by a software application which proactively harnesses the technology used for multimedia entertainment systems, rather than placing this burden on the user. 


\section{References}

Adobe. (2000). Interlaced and non-interlaced video. Retrieved from Adobe Premiere Manual:

http://www.indiana.edu/ aainfo/DigitalVideo/Info/Adobe/prmr_interlace.pdf

Amerasinghe, K. (2009, November 17). H.264 for the rest of us. Retrieved from Adobe:

http://wwwimages.adobe.com/www.adobe.com/content/dam/Adobe/en/devne t/video/articles/h264_primer/h264_primer.pdf

Axis Communications. (2008). H.264 video compression standard. Retrieved from http://www.wavestore.com/download.php?white_paper=2

Bainbridge, D., \& Novak, B. (2010). Seamless web editing for curated content. Proceedings of the 14th European conference on Research and advanced technology for digital libraries (pp. 168-175). Glasgow: Springer-Verlag .

Bainbridge, D., Jones, S., Mcintosh, S., Witten, I. H., \& Jones, M. (2008). Beyond the ClientServer Model: Self-contained Portable Digital Libraries. Proceedings of the 11th International Conference on Asian Digital Libraries: Universal and Ubiquitous Access to Information (pp. 294-303). Bali: Springer-Verlag.

Bainbridge, D., Novak, B., \& Cunningham, S. J. (2010). A user-centered design of a personal digital library for music exploration. Proceedings of the 10th annual joint conference on Digital libraries (pp. 149-158). Queensland: ACM.

Bolle, R. M., Yeo, B.-L., \& Yeung, M. M. (1998). Video query: Research directions. IBM Journal of Research and Development, pp. 233-252.

Bollen, J. (2007). Usage derived recommendations for a video digital library. Journal of network and computer applications, 1059-1083.

Card, S., Robertson, G., \& Mackinlay, J. (1991). The information visualize; an information workspace. Proceedings of SIGCHI Conference on Human Factors in Computing Systems, (pp. 181-188). New Orleans. 
Chang, S.-F., Smith, J. R., Meng, H. J., Wang, H., \& Zhong, D. (1997). Finding Images/Video in Large Archives. Columbia, USA.

Christel, M., \& Martin, D. (1998). Information Visualization Within a Digital Video Library . Journal of intelligent information systems, 235-257.

Christel, M., Kanade, T., Mauldin, M., Reddy, R., Sirbu, M., Stevens, S., \& Wactlar, H. (1995). Informedia Digital Video Library. Communications of the ACM, 57-58.

Christel, M., Stevens, S., Kanade, T., Mauldin, M., Reddy, R., \& Wactlar, H. (1996).

Techniques for the Creation and Exploration of Digital Video Libraries. Multimedia Tools and Applications.

Cotter, P., \& Smyth, B. (2000). PTV: Intelligent Personalised TV Guides. Proceedings of the Seventeenth National Conference on Artificial Intelligence and Twelfth Conference on Innovative Applications of Artificial Intelligence (pp. 957-964). AAAI Press.

Cunningham, S. J., \& Nichols, D. M. (2008). How people find videos. Proceedings of the 8th ACM/IEEE-CS joint conference on Digital libraries (pp. 201-210). Pittsburgh: ACM.

Dickson, G. (2009). Comedy Central Scans Airwaves with SnapStream. Broadcasting \& Cable.

Digital Video Broadcasting. (2011). DVB - Digital Video Broadcasting - New Zealand. Retrieved from DVB:

http://www.dvb.org/about_dvb/dvb_worldwide/new_zealand/index.xml

Ding, W., Marchionini, G., \& Soergel, D. (1999). Multimodal surrogates for video browsing. Proceedings of Digital Libraries '99. the fourth annual ACM conference on digital libraries (pp. 85-93). Berkeley: ACM.

Educational Recording Agency. (2011). The ERA Licensing Scheme. Retrieved from ERA: http://www.era.org.uk/

Elsweiler, D., Mandl, S., \& Kirkegaard Lunn, B. (2010). Understanding casual-leisure information needs: a diary study in the context of television viewing. IIIX '10 Proceeding of the third symposium on Information interaction in context (pp. 2534). New York: ACM. 
Enser, P., \& Sandom, C. (2002). Retrieval of archival moving imagery: CBIR outside the frame? Proceedings of the International Conference on Image and Video Retrieval (pp. 206-214). Berlin: Springer.

Environmental Systems Research Institute, Inc. (1999). MapObjects and ArcView. Retrieved from www.esri.com

Federal Communications Commission. (2011). Closed Captioning. Retrieved from FCC: http://www.fcc.gov/guides/closed-captioning

Fenwick, A. (2010). Stream of Consciousness, SnapStream and the future of searchable video. The News Frontier.

Fidel, R. (1997). The image retrieval task: Implications for design and evaluation of image databases. New Review of Hypermedia and Multimedia, 181-199.

Foley, J. (1997). Video indexing via speech recognition. InformationWeek, 104.

Geisler, G., Giersch, S., McArthur, D., \& McClelland, M. (2002). Creating Virtual Collections in Digital Libraries: Benefits and Implementation Issues. Proceedings of the Joint Conference on Digital Libraries (JCDL 2002), (pp. 210-218).

Grossmman, D. A., \& Frieder, O. (1998). Algorithms and heuristics. Boston: Kluwer Academic Publishers.

HDTVTunerinfo.com. (2010). Windows 7 Media Center TV Frequently Asked Questions. Retrieved from HDTV Tuner Info: http://www.hdtvtunerinfo.com/win7hdtvfaq.html

Honovich, J. (2008, June 14). Introduction to Video CODECS : MJPEG, MPEG-4, H.264. Retrieved from http://ipvideomarket.info/report/introduction_to_video_codecs_mjpeg_mpeg4_ h264

Hybrid Television Services (ANZ) Pty Limited. (2011). Frequently Asked Questions. Retrieved from My TiVo: http://www.mytivo.co.nz/faq/

International Telecommunication Union. (2010). Information technology - Generic coding of moving pictures and associated audio information: Systems. Retrieved 
from IUT: http://www.itu.int/dms_pubrec/itu-t/rec/h/T-REC-H.222.0-200605I!!SUM-HTM-E.htm

Ioannou, S., Moschovitis, G., Ntalianis, K., Karpouzis, K., \& Kollias, S. (2000). Effective access to large audiovisual assets based on user preferences. Proceedings of the 2000 ACM workshops on multimedia (pp. 227-232). Los Angeles: ACM.

Jang, P., \& Hauptmann, A. (1999). Learning to recognize speech by watching television. IEEE Intelligent Systems 14, 51-58.

Jones, P. (2001). Open(source)ing the doors for contributor-run digital libraries. 45.

Komlodi, A., \& Marchionini, G. (1998). Key frame preview techniques for video browsing. Proceedings of ACM DL '98 (pp. 24-26). Pittsburgh: ACM.

Kommission für Zulassung und Aufsicht. (2009). auf dem weg in die digitale welt: Rundfunk und internet waschen zusammen. Berlin, Germany.

Kordia (NZ) Limited. (2008). DIGITAL TERRESTRIAL TELEVISION LAUNCH IN NEW ZEALAND. Retrieved from Spectrum: http://www.kordia.co.nz/LiteratureRetrieve.aspx?ID=46031

Kordia (NZ) Ltd. (2008). Digital Terresterial TV (DTT) Project. Retrieved from http://www.itu.int/dms_pubrec/itu-t/rec/h/T-REC-H.222.0-200605-I!!SUMHTM-E.htm

Kubala, F., Schwartz, R., Stone, R., \& Weischedel, R. (1998). Named entity extraction from speech. Proceedings of DARPA Broadcast News Workshop.

Kukulska-Hulme, A., Van Der Zwan, R., Dipaolo, T., Evers, V., \& Clarke, S. (1999). An evaluation of the Informedia Digital Video Library System at the Open University. Learning, media and technology, 131-136.

Levy, C. (2010). Future of television: Online and on-demand. Toronto star.

Ludwig, B., \& Mandl, S. (2010). Centering information retrieval to the user. Revue d'Intelligence Artificielle 24, pp. 95-108. 
Marchionini, G. (1995). Information seeking in electronic environments. New York: Cambridge U. Press.

Marchionini, G. (1999). Augmenting library services: Toward the sharium. Proceedings of International Symposium on Digital Libraries 1999, (pp. 40-47). Tsukuba.

Marchionini, G. (2004). A briefing on the evolution and status of the Open Video digital library . International journal on digital libraries, 36-38.

Marchionini, G. (2006). The Open Video Digital Library: A Möbius strip of research and practice. Journal of the American Society for Information Science and Technology, 1626-1643.

Marchionini, G., \& Geisler, G. (2002). The Open Video Digital Library. D-Lib Magazine.

Marchionini, G., Geisler, G., \& Brunk, B. (2000). Agileviews: A Human-Centered Framework for Interfaces to Information Spaces. Proceedings of the Annual Meeting of the American Society for Information Science, (pp. 271-280). Chicago.

Marchionini, G., Nolet, V., Williams, H., Ding, W., Beale, J., Rose, A., \& Gordon, A. (1997). Content + Connectivity => Community: Digital Resources for a Learning Community. Proceeding of ACM Digital Libraries 97 (pp. 212-220). Philadelphia: ACM.

Marcos, M.-C., Marchionini, G., \& Russell, T. (2008). User Scenarios in the Open Video Digital Library Redesign. IX Congreso Internacional Interacción PersonaOrdenador, (pp. 127-130). Albacete.

McCandless, M., Hatcher, E., \& Gospodnetic, O. (2010). Lucene in Action Second Edition. Stamford: Manning Publications.

Microsoft Corporation. (2009). Record TV in Windows Media Center. Retrieved October 8, 2009, from Windows Vista - Microsoft Windows: http://windows.microsoft.com/en-us/windows-vista/Record-TV-in-WindowsMedia-Center

Microsoft Corporation. (2011). What should I know before adding TV tuners to use with Windows Media Center. Retrieved from Windows: 
http://windows.microsoft.com/en-US/windows-vista/What-should-I-knowbefore-adding-TV-tuners-to-use-with-Windows-Media-Center

Muniz de Ávila, P., \& Donizetti Zorzo, S. (2009). A personalized TV guide system: an approach to interactive digital television. SMC'09 Proceedings of the 2009 IEEE international conference on Systems, Man and Cybernetics (pp. 2122-2127). New York: ACM.

Nahrstedt, W. (2000). Global edutainment: the role of leisure education for community development. Leisure education, 65-74.

New Zealand Digital Library Project. (2011). About Greenstone. Retrieved from Greenstone Digital Library Software: http://www.greenstone.org/

Nichols, D. M., Bainbridge, D., \& Twidale, M. B. (2007). Constructing digital library interfaces. Proceedings of the 7th ACM/IEEE-CS joint conference on Digital libraries (pp. 331-332). Vancouver: ACM.

Nielsen, J. (1993). Usability Engineering. San Francisco: Morgan Kaufmann.

Patel, P. (2009). Local company is early to video search technology. Houston Chronicle.

Paula Wilson, A. (2005, Jul/Aug). Digital Video Recording: Personalized Television. Public Libraries, pp. 210-212.

Planet Enterprises Ltd. (2008). Planet eStream User Stories \& Testimonials. Retrieved from Planet eStream v4.5: http://v45.planetestream.co.uk/testimonials.aspx

Planet Enterprises Ltd. (2011). Education brochure 2011.

Planet Enterprises Ltd. (2011). Educational Benefits. Retrieved from Planet eStream: http://www.planetestream.co.uk/Benefits.aspx

Planet Enterprises Ltd. (2011). Freeview Recording Module. Retrieved from Planet eStream: http://www.planetestream.co.uk/module_freeview.aspx

Planet Enterprises Ltd. (2011). PLANET AV finance. Retrieved from Planet DV: http://www.planetdv.net/Finance.html 
Planet PC. (2011). Freeview Admin Features . Retrieved from Planet eStream Tech Blog: http://planetestream.blogspot.com/2011/07/new-freeview-administrativefeatures.html

Rasmussen, E. (1997). Indexing images. Annual Review of Information Science and Technology, 169-196.

Roüast, M. (2010). Television in a Digital Library. Hamilton: The University of Waikato.

Sabbagh, D. (2007). BBC joins the rush to provide internet television on demand. The Times, 13.

Schraefel, M. C., Smith, D. A., Owens, A., Russel, A., Harris, C., \& Wilson, M. (2005). The evolving mSpace platform: leveraging the semantic web on the trail of the memex. Proceedings of the sixteenth ACM conference on Hypertext and hypermedia (pp. 174-183). Austria: ACM.

Shneiderman, B. (1994). Dynamic queries for visual information seeking. IEEE Software, 70-77.

Shyu, M.-L., Chen, S.-C., Chen, M., Zhang, C., \& Sarinnapakorn, K. (2003). Image database retrieval utilizing affinity relationships. Proceedings of the 1st ACM international workshop on Multimedia databases (pp. 78-85). Los Angeles: ACM.

Sky. (2011). Using your Sky+HD Planner . Retrieved from Sky:

http://www.sky.com/helpcentre/tv/sky-plus-hd/recording/sky-guide-plannerhd/

Slaughter, L., Marchionini, G., \& Geisler, G. (2000). Open Video: A Framework for a Test Collection. Journal of Network and Computer Applications, Special Issue On Network-Based Storage Services, 219-245.

Snap Stream. (2011). Pricing. Retrieved from SnapStream TV Search: http://www.snapstream.com/enterprise/buynow/pricing.asp

Snap Stream. (2011). Snap Stream HD. Retrieved from SnapStream TV Search: http://www.snapstream.com/enterprise/HD.asp 
Sun, J., Wang, Z., Yu, H., Nishino, F., Katsuyama, Y., \& Naoi, S. (2003). Effective text extraction and recognition for WWW images. Proceedings of the 2003 ACM symposium on Document engineering (pp. 115-117). Grenoble: ACM.

Tech Talks. (2010). MySky HDi v TiVo. Retrieved from http://nz.news.yahoo.com/opinion/post/-/blog/techtalks/post/154/comment/

TiVo. (2009). International TiVo DVR \& TiVo service. Retrieved October 8, 2009, from TiVo: http://www.tivo.com/abouttivo/international/index.html

TiVo. (2009). TiVo HD and TiVo HD XL Viewer's Guide - Chapter 1. Retrieved October 7, 2009, from TiVo: http://support.tivo.com/ci/fattach/get/33/1244847724/redirect/1

TNS Gallup. (2010). TNS gallup tv-meter.

Tse, T., Marchionini, G., Ding, W., Slaughter, L., \& Komlodi, A. (1998). Dynamic key frame presentation techniques for augmenting video browsing. Proceedings of AVI '98: Advanced Visual Interfaces (pp. 185-194). L' Aquila: ACM.

Tuitfun. (2011). movie thumbnailer (mtn) usage. Retrieved from http://moviethumbnail.sourceforge.net/usage.en.html

VideoLAN. (2011). Deinterlacing. Retrieved from VideoLAN Wiki: wiki.videolan.org/Deinterlacing

Wactlar, H., Christel, M., Gong, Y., \& Hauptmann, A. (1999). Lessons Learned from Building a Terabyte Digital Video Library. Computer, 66-73.

Wactlar, H., Kanade, T., \& Smith, M. S. (1996). Intelligent Access to Digital Video: Informedia Project. Computer, 46-52.

Wactlar, H., Kanade, T., Smith, M., \& Stevens, S. (1996). Intelligent Access to DigitalVideo: Informedia Project. Computer, 46-52.

Wactlarv, H. D., Hauptmann, A. G., Christel, M. O., Houghton, R. A., \& Olligschlaeger, A. M. (2000). Complementary Video and Audio Analysis for Broadcast News Archives. Communications of the ACM, 42-47. 
Witbrock, M. J., \& Hauptmann, A. G. (1998). Speech Recognition for a Digital Video Library. Journal of the American Society for Information Science, 619-632.

Witten, I. H. (2004). Creating and customizing digital library collections with the Greenstone Librarian Interface. Proceedings of International Symposium on Digital Libraries and Knowledge Communities in Networked Information Society (pp. 97 104). Tokyo: University of Tsukuba.

Witten, I., Bainbridge, D., \& Nichols, D. (2010). How to Build a Digital Library Second Edition. Burlington: Morgan Kaufmann.

Witten, I., Moffat, A., \& Bell, T. (1999). Managing Gigabytes: Compressing and Indexing Documents and Images Second Edition. San Francisco: Morgan Kaufmann.

Yang, M., Mu, X., \& Marchionini, G. (2003). Vivo-a video indexing and visualization organizer. Proceedings of the Third ACM/IEEE-CS Joint Conference on Digital Libraries (p. 403). ACM.

Yee, K.-P., Swearingen, K., Li, K., \& Hearst, M. (2003). Faceted metadata for image search and browsing. Proceedings of the SIGCHI conference on Human factors in computing systems (pp. 401-408). Florida: ACM.

Zhang, H., Smoliar, S. W., Wu, J. H., Low, C. Y., \& Kankanhalli, A. (1995). A Video Database System for Digital Libraries. Selected Papers from the Digital Libraries Workshop on Digital Libraries: Current Issues , 253-264.

Zimmerman, J. (2004). Personalized digital television: Targeting programs to individual viewers. Design of a TV Show Recommender Engine and Interface, pp. 27-51. 